International Journal of Modern Physics A, (C) World Scientific Publishing Company

OUNP-2001-03

\title{
AN EXPERIMENTALIST'S VIEW OF NEUTRINO OSCILLATIONS
}

\author{
ANTONELLA DE SANTO \\ Sub-Department of Nuclear and Particle Physics, University of Oxford, 1 Keble Road \\ Oxford, OX1 3RH, United Kingdom
}

Invited paper to appear in Intern. J. Mod. Phys. A (2001)

\begin{abstract}
Neutrinos, and primarily neutrino oscillations, have undoubtedly been one of the most exciting topics in the field of high-energy physics over the past few years. The existence of neutrino oscillations would require an extension of the currently accepted description of sub-nuclear phenomena beyond the Standard Model. Compelling evidence of new physics, which seems to be pointing towards neutrino oscillations, is coming from the solar neutrino deficit and from the atmospheric neutrino anomaly. More controversial effects have been observed with artificially produced neutrinos. The present experimental status of neutrino oscillations is reviewed, as well as the planned future experimental programme, which, it is hoped, will solve most of the outstanding puzzles.
\end{abstract}

\section{Introduction}

Since the discovery of the electron neutrino in 1955t, the study of neutrino physics has often led to great progress in the unravelling of sub-nuclear phenomena. However, although they have been used as powerful probes of elementary particles interactions, very little is known about the general properties of neutrinos: we know neither their mass, nor their magnetic moment; we don't know whether they are Dirac or Majorana particles; we don't know how many neutrino species exist in nature. In many respects, neutrinos perhaps remain the most intriguing mystery in particle physics.

In more recent years most of the research activity in neutrino physics has focused on the phenomenon of neutrino oscillations, originally postulated by Bruno Pontecorvo. 2 Interest in this field has been revived by several very interesting experimental results, which in some cases very strongly support the hypothesis of neutrino oscillations. This is only possible if, contrary to what assumed in the Standard Model of electro-weak interactions, neutrinos have non-zero and non-degenerate masses. Thus, evidence for neutrino oscillations would represent a major discovery in elementary particle physics and would then require a complete revolution in our understanding of the natural world.

This is a very exciting time for neutrino physics, with new data becoming available in a relatively short period of time. Whilst this paper was being completed, the long-awaited first results from the SNO experiment were presented. that many others will follow, from all the running or planned experiments, and that more data will soon lead to the solution of this fascinating puzzle of particle physics. 
In this paper, after a brief introduction to massive neutrinos and to the formalism of neutrino oscillations, the latest experimental results will be reviewed. The focus will be on those experiments which have added pieces of information to the current picture of neutrino oscillation phenomenology, either by observing a signal or by setting a limit in an interesting portion of the relevant parameter space. Planned experiments, designed to resolve some of the as yet unsolved ambiguities, will also be described.

\section{Neutrino Masses}

The present experimental limits on neutrino masses, as obtained from direct kinematic searches, under the assumption that flavour and mass eigenstates are essentially coincident, are the following 4.6 .6.

$$
\begin{array}{ll}
m_{\nu_{e}}<2.2 \mathrm{eV} & \left(95 \% \text { C.L., from }{ }^{3} \mathrm{H} \rightarrow{ }^{3} \mathrm{He}+e^{-}+\bar{\nu}_{e}\right) \\
m_{\nu_{\mu}}<170 \mathrm{keV} & \left(90 \% \text { C.L., from } \pi^{+} \rightarrow \mu^{+}+\nu_{\mu}\right) \\
m_{\nu_{\tau}}<15.5 \mathrm{MeV} & \left(95 \% \text { C.L., from } \tau \rightarrow 5 \pi\left(\pi^{0}\right)+\nu_{\tau}\right) .
\end{array}
$$

Although the smallness of these limits might lead to the naive conclusion that neutrino masses are exactly vanishing, there is no theoretical reason to assume that this is indeed the case. On the contrary, it is generally believed that particle masses can be identically equal to zero only if they are associated with an exact gauge symmetry. This is not true for the lepton quantum number, which is then expected not to be conserved.

Perhaps the most popular expression for the neutrino mass term is the one associated with the so-called see-saul form of the mass matrix. In the simple case of one generation this mass term is given by:

$$
\left(\begin{array}{cc}
\nu_{L}, & \nu_{R}
\end{array}\right)\left(\begin{array}{cc}
0 & m_{D} \\
m_{D} & M_{M}
\end{array}\right)\left(\begin{array}{c}
\nu_{L} \\
\nu_{R}
\end{array}\right)
$$

where a right-handed neutrino singlet, $\nu_{R}$, has been introduced. Assuming that the Dirac mass $m_{D}$ is much smaller than the Majorana mass $M_{M}$, the diagonalisation of (2) gives a heavy eigenstate, of mass $\sim M_{M}$, and a light eigenstate, which can be identified with the standard left-handed neutrino and whose mass is given by:

$$
m_{\nu} \simeq \frac{m_{D}^{2}}{M_{M}} .
$$

If the Dirac mass is of the same order as that of a fundamental fermion, be it a quark or a charged lepton $\left(m_{D} \sim O\left(m_{q, \ell}\right)\right)$, and if $M_{M} \sim \mathrm{O}\left(M_{G U T}\right)$, then the neutrino mass turns out to be naturally very small, and in a range of values compatible with those indicated by experimental results.

$\overline{a_{\text {in }} \text { the generic } n \text {-generation case } m_{D}}$ and $M_{M}$ are $n \times n$ matrices: then $m_{D} \ll M_{D}$ means that all eigenvalues of $m_{D}$ are small compared to all eigenvalues of $M_{M}$. 
Several versions of the see-saw mechanism are considered in literature 1 Among them, the so-called quadratic see-saw, in which all the Majorana masses are similar to each other, and therefore the light neutrino masses scale as the Dirac masses squared:

$$
m_{\nu_{1}}: m_{\nu_{2}}: m_{\nu_{3}} \simeq m_{q_{1}, \ell_{1}}^{2}: m_{q_{2}, \ell_{2}}^{2}: m_{q_{3}, \ell_{3}}^{2}
$$

Another possibility is the linear see-saw, where $M_{M} \propto m_{D}$ and then:

$$
m_{\nu_{1}}: m_{\nu_{2}}: m_{\nu_{3}} \simeq m_{q_{1}, \ell_{1}}: m_{q_{2}, \ell_{2}}: m_{q_{3}, \ell_{3}}
$$

Both versions imply a hierarchy between the light neutrino mass eigenstates:

$$
m_{\nu_{1}} \ll m_{\nu_{2}} \ll m_{\nu_{3}} .
$$

\section{Neutrino Oscillations in Vacuum}

Ordinary neutrinos are produced in weak processes together with their charged partner leptons $\left(W^{+} \rightarrow \ell^{+} \nu_{\ell}\right)$, as for example in pion decays or in nuclear beta decays, and they are therefore eigenstates of the weak interactions. On the other hand, weak interaction eigenstates and mass eigenstates are not necessarily coincident. With a change of basis, neutrinos produced as flavour eigenstates can be represented as a coherent linear superposition of the mass eigenstates:

$$
\left|\nu_{\alpha}\right\rangle=\sum_{i} U_{\alpha i}^{*}\left|\nu_{i}\right\rangle \quad(\alpha=e, \mu, \tau)
$$

where $i$ runs over all the existing mass eigenstates and $U_{\alpha i}$ are the elements of a unitary matrix. ${ }^{b}$

Although from measurements of the $Z^{0}$ width at LEP we know that the number of light active neutrinos is $\mathcal{N}_{\nu}=2.994 \pm 0.012$, nothing forbids the existence of more than three neutrinos of definite mass. If, for example, there were four independent mass eigenstates, a change of basis from the mass to the flavour eigenstates would necessarily lead to one linear combination,

$$
\left|\nu_{s}\right\rangle=\sum_{i} U_{s i}^{*}\left|\nu_{i}\right\rangle
$$

which has no normal weak coupling. The linear combination (8) is therefore referred to as a "sterile neutrino", as opposed to the standard "active" neutrino.

As we shall see in the next sections, the existence of at least one sterile neutrino might be the only way to accommodate all the experimental results on neutrino oscillations. Nonetheless, for the sake of simplicity, in the following discussion we will only consider the three neutrino flavours whose existence has been firmly

\footnotetext{
${ }^{b}$ The convention of using Greeks letters to identify flavour eigenstates and Latin letters to identify mass eigenstatates has been adopted throughout this paper.
} 
An Experimentalist's View of ...

established. ${ }^{c}$ However, it must be noted that the same formalism would be valid for any number of neutrinos.

After a neutrino has been prepared in the state $\left|\nu_{\alpha}\right\rangle$ at $t=0$, each of its components will evolve according to the Schrödinger equation. At a time $t$ after production it will be:

$$
\left|\nu_{\alpha}(t>0)\right\rangle=\sum_{i} e^{-i E_{i} t} U_{\alpha i}^{*}\left|\nu_{i}\right\rangle=\sum_{\beta} \sum_{i} U_{\beta i} e^{-i E_{i} t} U_{\alpha i}^{*}\left|\nu_{i}\right\rangle
$$

Thus, after the neutrino produced in the flavour eigenstate $\alpha$ has travelled a distance $L \simeq t$, the probability of finding it in a different flavour state $\beta$ is non-zero and is given by:

$$
\mathcal{P}\left(\nu_{\alpha} \rightarrow \nu_{\beta}\right)=\left|\left\langle\nu_{\beta}(t) \mid \nu_{\alpha}\right\rangle\right|^{2}=\left|\sum_{i} U_{\beta i} e^{-i E_{i} t} U_{\alpha i}^{*}\right|^{2}
$$

Using the unitarity condition for $U$, after some algebra the probability (10) takes the following oscillatory form:

$$
\mathcal{P}\left(\nu_{\alpha} \rightarrow \nu_{\beta}\right)=\sum_{i}\left|U_{\beta i}\right|^{2}\left|U_{\alpha i}\right|^{2}+2 R e \sum_{j>i} U_{\beta i} U_{\beta j}^{*} U_{\alpha i}^{*} U_{\alpha j} \exp \left(-i \frac{\Delta m_{j k}^{2} L}{2 E}\right)
$$

where $\Delta m_{i j}^{2}=\left|m_{i}^{2}-m_{j}^{2}\right|$ and the ultra-relativistic expansion for the neutrino energy has been used. ${ }^{d}$ Thus the transition probability $\mathcal{P}\left(\nu_{\alpha} \rightarrow \nu_{\beta}\right)$ depends on a combination of the mixing matrix elements, on the squared difference between the mass eigenvalues of the mass eigenstates, and on two parameters, the neutrino energy $E$ and the distance $L$ between the production and the observation points, which are determined by the experimental conditions.

Although from the simplicity of the preceding discussion it might seem that neutrino oscillations can be obtained as a straightforward consequence of elementary quantum mechanics, a rigorous treatment of the problem would require that a wave packet formalism be considered. We shall not discuss this approach here and the interested reader is remanded, for example, to ref.11 and refs. therein.

The lepton mixing matrix $U$, also known as the Maki-Nakagawa-Sakata (MNS) matrix,12 is the leptonic analogue of the CKM matrix for the quark sector. In the case of three neutrino flavours the explicit relation between the flavour and the mass eigenstates is given by:

$$
\left(\begin{array}{c}
\nu_{e} \\
\nu_{\mu} \\
\nu_{\tau}
\end{array}\right)=\left(\begin{array}{ccc}
U_{e 1} & U_{e 2} & U_{e 3} \\
U_{\mu 1} & U_{\mu 2} & U_{\mu 3} \\
U_{\tau 1} & U_{\tau 2} & U_{\tau 3}
\end{array}\right)\left(\begin{array}{l}
\nu_{1} \\
\nu_{2} \\
\nu_{3}
\end{array}\right)
$$

\footnotetext{
${ }^{c}$ the existence of the $\tau$ neutrino, of which only indirect evidence watable until very recent times, has been proven by the emulsion-based DONUT experiment 10 at Fermilab.

${ }^{d} E_{i}=\sqrt{p^{2}+m_{i}^{2}} \simeq p+m_{i}^{2} / 2 p \simeq p+m_{i}^{2} / 2 E$.
} 
It is sometimes convenient to parametrize $U$ in a form analogous to that of the CKM matrix, 6 which for Dirac neutrinos is:

$$
U=\left(\begin{array}{ccc}
c_{12} c_{13} & s_{12} c_{13} & s_{13} e^{-i \delta} \\
-s_{12} c_{23}-c_{12} s_{23} s_{13} e^{i \delta} & c_{12} c_{23}-s_{12} s_{23} s_{13} e^{i \delta} & s_{23} c_{13} \\
s_{12} s_{23}-c_{12} c_{23} s_{13} e^{i \delta} & -c_{12} s_{23}-s_{12} c_{23} s_{13} e^{i \delta} & c_{23} c_{13}
\end{array}\right),
$$

with the standard convention $c_{i j}=\cos \theta_{i j}$ and $s_{i j}=\sin \theta_{i j}(i, j=1,2,3$ are the generation labels). Thus in this formalism the mixing matrix depends only on three mixing angles, $\theta_{12}, \theta_{13}$ and $\theta_{23}$, and a CP-violating phase, $\delta .{ }^{e}$

Although the most general description of the mixing among all neutrinos implies the three-flavour treatment, most experimental results are expressed in the two generation mixing representation. More recent analyses, however, generally attempt a global fit to all data assuming three-flavour mixing.

In the case of transitions between two flavours the mixing matrix has the form of a rotation in a two-dimensional space:

$$
U=\left(\begin{array}{cc}
\cos \theta & \sin \theta \\
-\sin \theta & \cos \theta
\end{array}\right)
$$

where $\theta$ is the mixing angle, analogous to the Cabibbo angle for the quarks. The relation between the flavour and the mass eigenstate is then given by

$$
\begin{aligned}
& \left|\nu_{\alpha}\right\rangle=\cos \theta\left|\nu_{1}\right\rangle+\sin \theta\left|\nu_{2}\right\rangle \\
& \left|\nu_{\beta}\right\rangle=-\sin \theta\left|\nu_{1}\right\rangle+\cos \theta\left|\nu_{2}\right\rangle
\end{aligned} .
$$

The oscillation probability in this case can be written as follows:

$$
\mathcal{P}\left(\nu_{\alpha} \rightarrow \nu_{\beta}\right)=\sin ^{2} 2 \theta \sin ^{2}\left(\pi \frac{L}{\lambda_{\text {osc }}}\right)
$$

where $\lambda_{o s c}$ is the oscillation wavelength, defined as:

$$
\lambda_{o s c}=\frac{4 \pi E}{\Delta m^{2}},
$$

where $E$ is again the neutrino energy and $\Delta m^{2}$ is the squared neutrino mass difference.

Substituting the expression of $\lambda_{\text {osc }}$ in (16), the oscillation probability takes its usual form:

$$
\mathcal{P}\left(\nu_{\alpha} \rightarrow \nu_{\beta}\right)=\sin ^{2} 2 \theta \sin ^{2}\left(\frac{1.27 \Delta m^{2} L}{E}\right),
$$

where $\Delta m^{2}$ is expressed in $e \mathrm{~V}^{2}$, the detector-to-source distance $L$ is in meters $(k \mathrm{~m})$ and the neutrino energy $E$ in $\mathrm{MeV}(\mathrm{GeV})$.

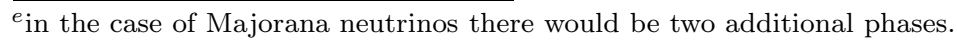


The oscillation probability 18 is the product of two factors. The first one, $\sin ^{2} 2 \theta$, does not depend on the experimental conditions, but is an intrinsic parameter which describes the strength of the coupling between the two neutrino flavour; it gives the maximum amplitude of the neutrino oscillations. The mixing is maximal when $\sin ^{2} 2 \theta=1$, namely when $\theta=\pi / 4$.

The second factor is another oscillatory term, the period of which is determined by $\Delta m^{2} L / E$. In order to have sensitivity to small values of $\Delta m^{2}$, the ratio $E / L$ must be also small. This can be achieved by having low energy neutrino beams and large distances between the neutrino source and the detector. On the other hand, when the oscillation phase becomes too large, that is to say when $E / L$ is too small compared to $\Delta m^{2}$, the oscillations occur very rapidly and, due to the finite energy resolution of the experiments, the term $\sin ^{2}\left(1.27 \Delta m^{2} L / E\right)$ averages to $1 / 2$.

Before concluding this overview of the phenomenology of neutrino oscillations in vacuum, it is interesting to consider the effect of the CPT, CP and T symmetries on the oscillation probabilities. Without entering in any details, we shall only say that for both Dirac and Majorana neutrinos, CPT invariance implies:

$$
\mathcal{P}\left(\nu_{\alpha} \rightarrow \nu_{\beta}\right)=\mathcal{P}\left(\bar{\nu}_{\beta} \rightarrow \bar{\nu}_{\alpha}\right) \quad(\text { CPT conserved }) .
$$

In particular, this means that the neutrino and the antineutrino survival probabilities are the same:

$$
\mathcal{P}\left(\nu_{\alpha} \rightarrow \nu_{\alpha}\right)=\mathcal{P}\left(\bar{\nu}_{\alpha} \rightarrow \bar{\nu}_{\alpha}\right) \quad(\text { CPT conserved }) .
$$

On the other hand, the oscillation probabilities for neutrinos and antineutrinos are generally different. They coincide only if CP, the symmetry which converts a left-handed neutrino into a right-handed antineutrino, is conserved:

$$
\mathcal{P}\left(\nu_{\alpha} \rightarrow \nu_{\beta}\right)=\mathcal{P}\left(\bar{\nu}_{\alpha} \rightarrow \bar{\nu}_{\beta}\right) \quad(\text { CP conserved }) .
$$

Finally, if the time-reversal symmetry $\mathrm{T}$ is conserved, the oscillation probability is invariant under interchange of the initial and the final states:

$$
\mathcal{P}\left(\nu_{\alpha} \rightarrow \nu_{\beta}\right)=\mathcal{P}\left(\nu_{\beta} \rightarrow \nu_{\alpha}\right) \quad(\text { T conserved }) .
$$

\section{Neutrino Oscillations in Matter}

In the previous section we have seen that the probability for neutrino oscillations in vacuum cannot exceed $\sin ^{2} 2 \theta, \theta$ being the mixing angle in vacuum between the two flavours under consideration. On the contrary, the situation can be very different for neutrino oscillations in matter. Matter effects can greatly enhance neutrino mixing, resulting in a large oscillation probability even for very small vacuum mixing angles.

When they travel through matter, neutrinos of all flavours can have neutralcurrent interactions with the protons, neutrons and electrons of the medium. However only electron neutrinos can interact with the electrons, undergoing a coherent 
forward scattering via a $W$ boson exchange. The consequence of this asymmetry between neutrino flavours is known as the Mikheyev-Smirnov-Wolfenstein (MSW) effect.13, 44

At low neutrino energies, for electron, muon and tau neutrinos traversing an electrically neutral and unpolarised medium, the matter-induced potentials are given by:

$$
\begin{gathered}
V_{e}=\sqrt{2} G_{F}\left(\mathcal{N}_{e}-\frac{\mathcal{N}_{n}}{2}\right) \\
V_{\mu}=V_{\tau}=-\sqrt{2} G_{F} \frac{\mathcal{N}_{n}}{2},
\end{gathered}
$$

where $G_{F}$ is the Fermi constant and $\mathcal{N}_{e}$ and $\mathcal{N}_{n}$ are the electron and the neutron number densities respectively. For antineutrinos the potentials have opposite signs.

For simplicity, the discussion will be restricted to the two-flavour scenario, more specifically to the mixing between $\nu_{e}$ and $\nu_{\mu}$. In this case, using the ultra-relativistic expansion for the neutrino energy and neglecting all common phases (which would not affect the result for mixing between active neutrinos), the time evolution for the flavour eigenstates is given by:

$$
i \frac{d}{d t}\left(\begin{array}{c}
\nu_{e} \\
\nu_{\mu}
\end{array}\right)=\left(\begin{array}{cc}
-\frac{\Delta m^{2}}{4 E} \cos 2 \theta+\sqrt{2} G_{F} \mathcal{N}_{e} & \frac{\Delta m^{2}}{4 E} \sin 2 \theta \\
\frac{\Delta m^{2}}{4 E} \sin 2 \theta & \frac{\Delta m^{2}}{4 E} \cos 2 \theta
\end{array}\right)\left(\begin{array}{c}
\nu_{e} \\
\nu_{\mu}
\end{array}\right),
$$

where the common term due to neutral-current interactions, proportional to the identity matrix, has been ignored.

The simplest case of matter distribution is that of constant matter density, $\mathcal{N}_{e}=$ const. Although not very realistic, this is however quite an instructive example and we shall discuss it here in some detail.

As for the mass and flavour eigenstates for oscillations in vacuum, the mass and flavour eigenstates in matter are also connected by a two-dimensional rotation. The relation between the two bases is given by:

$$
\begin{aligned}
& \left|\nu_{1}^{M}\right\rangle=\cos \theta_{M}\left|\nu_{e}\right\rangle+\sin \theta_{M}\left|\nu_{\mu}\right\rangle \\
& \left|\nu_{2}^{M}\right\rangle=-\sin \theta_{M}\left|\nu_{e}\right\rangle+\cos \theta_{M}\left|\nu_{\mu}\right\rangle .
\end{aligned}
$$

The vacuum mixing angle, $\theta$, has been replaced by the mixing angle in matter, $\theta_{M}$, given by:

$$
\sin ^{2} 2 \theta_{M}=\frac{\left(\frac{\Delta m^{2}}{2 E}\right)^{2} \sin ^{2} 2 \theta}{\left(\frac{\Delta m^{2}}{2 E} \cos 2 \theta-\sqrt{2} G_{F} \mathcal{N}_{e}\right)^{2}+\left(\frac{\Delta m^{2}}{2 E}\right)^{2} \sin ^{2} 2 \theta} .
$$

It has to be noticed that, since the mixing angle is not the same as in vacuum, the matter eigenstates $\left|\nu_{1}^{M}\right\rangle$ and $\left|\nu_{2}^{M}\right\rangle$ are not coincident with the mass eigenstates in vacuum, $\left|\nu_{1}\right\rangle$ and $\left|\nu_{2}\right\rangle$. 
The oscillation probability is of the same form as in Eq.(16), but the vacuum mixing angle and the vacuum oscillation wavelength are now replaced by those in matter:

$$
\mathcal{P}\left(\nu_{e} \rightarrow \nu_{\mu}\right)=\sin ^{2} 2 \theta_{M} \sin ^{2}\left(\pi \frac{L}{\lambda_{M}}\right)
$$

with $\lambda_{M}$ given by:

$$
\lambda_{M}=\frac{2 \pi}{\sqrt{\left(\frac{\Delta m^{2}}{2 E} \cos 2 \theta-\sqrt{2} G_{F} \mathcal{N}_{e}\right)^{2}+\left(\frac{\Delta m^{2}}{2 E}\right)^{2} \sin ^{2} 2 \theta}} .
$$

From Eq.(26) it follows that, regardless of the smallness of the mixing angle in vacuum, the mixing angle in matter can be very large. In particular, maximal mixing can be achieved if the medium density is such that the following condition is satisfied:

$$
\sqrt{2} G_{F} \mathcal{N}_{e}=\frac{\Delta m^{2}}{2 E} \cos 2 \theta .
$$

This is the the so-called $M S W$ resonance condition. Using the characteristic solar electron density, $\mathcal{N}_{e} \sim 10^{26} \mathrm{~cm}^{-3}$, and the characteristic value for solar neutrino energies, $E \sim 1 \mathrm{MeV}$, assuming small mixing in vacuum (hence $\cos 2 \theta \sim 1$ ), one obtains $\Delta m^{2} \sim 10^{-5} \mathrm{eV}^{2}$.

Since $\mathcal{N}_{e}>0$, Eq. (29) is fulfilled only if $\Delta m^{2} \cos \theta>0$. Once a convention on the phase has been chosen, Eq. 29) implies that resonant oscillation enhancement is possible only for one particular sign of $\Delta m^{2}$. For example, if $\cos \theta>0$, neutrino oscillations are enhanced if $\Delta m^{2}=m_{2}^{2}-m_{1}^{2}>0$. On the other hand, from the change of sign in the matter-induced potentials, it follows that for antineutrinos the resonance condition would require $\Delta m^{2}<0$. This means that, for a given sign of $\Delta m^{2}$, matter effects cannot enhance neutrino and antineutrino oscillations at the same time: if neutrino oscillations are enhanced, antineutrino oscillations will be suppressed, and vice-versa.

The evolution equation (24) cannot be solved analytically for any non-uniform matter distribution. In general a numerical solution of the system of differential equations (24) has to be computed, accounting for the density profile and the neutrino energy distribution. An interesting case to consider is the so-called adiabatic approximation for matter density monotonically decreasing along the neutrino path. Without entering into the details of the calculation, we shall only qualitatively discuss the results for the case of two-flavour neutrino mixing (for example between $\nu_{e}$ and $\nu_{\mu}$ ), which is useful to describe neutrino oscillations in the Sun.

Electron neutrinos produced in the core of the Sun, where the density is well above that corresponding to the MSW resonance, will initially see a mixing angle $\theta_{\text {core }} \approx \pi / 2$. From Eq. (25) it follows that, at production point, a neutrino born as a $\nu_{e}$ will essentially coincide with one of the matter eigenstates $\nu_{2}^{M}$. The adiabaticity condition, that requires slowly changing matter distribution, guarantees that the 
neutrino system can gradually adjust to the changing density of the environment and therefore the system will not make any transition to the other matter eigenstate. As $\nu_{2}^{M}$ propagates through the mantle, it encounters regions of smaller densities: the effective mixing angle decreases and the strength of the mixing increases, until the resonance condition is fulfilled and maximal mixing is reached. As the neutrino travels further, the mixing angle becomes smaller and smaller, approaching the value of the mixing angle in vacuum, $\theta$. If $\theta$ is very small, the $\nu_{e}$ component of $\nu_{2}^{M}$ is very small at the final point and $\nu_{2}^{M}$ is mainly composed of $\nu_{\mu}$. The survival and the oscillation probabilities for electron neutrinos are given by:

$$
\begin{aligned}
& \mathcal{P}\left(\nu_{e} \rightarrow \nu_{e}\right)=\sin ^{2} \theta \\
& \mathcal{P}\left(\nu_{e} \rightarrow \nu_{\mu}\right)=\cos ^{2} \theta .
\end{aligned}
$$

Thus, in the range of validity of the adiabatic approximation and for small values of the mixing angle in vacuum, if the depth of the traversed matter is large enough, the probability of finding the neutrino in the flavour state $\nu_{e}$ when it gets outside the Sun is tiny and a complete conversion of $\nu_{e}$ to $\nu_{\mu}$ is possible.

At this point it becomes natural to discuss the solar neutrino deficit and to present the status of the search for neutrino oscillations in the Sun.

\section{The Solar Neutrino Problem}

\subsection{Solar Models}

Historically the first hint for neutrino oscillations came from the observation of neutrinos from the Sun. In 1968 Ray Davis and his collaborators published the first results of the Homestake chlorine experiment, 15.16 showing that the measured flux of solar electron neutrinos was significantly lower than the expected value. This was the beginning of the solar neutrino deficit saga.

The Homestake results were later confirmed by four other experiments: SAGE 17 GALLEX,18 Kamiokande 19 and Super-Kamiokande 20 Both SAGE and GALLEX are radiochemical experiments, like Homestake, while Kamiokande and Super-Kamiokande are water-Cherenkov experiments. Before we discuss them, it is important to introduce the so-called Standard Solar Model (SSM), used to predict the solar neutrino flux under the assumption that no exotic phenomena, such as neutrino oscillations, can affect the nature of the produced neutrinos before they reach the Earth.

Our Sun belongs to the category of the main sequence stars, which produce energy in their interiors via thermonuclear reactions. The two reaction chains responsible for the energy production are known as the pp-cycle and the CNO-cycle. For both cycles the overall result is the fusion of hydrogen nuclei into helium, with the emission of electron neutrinos:

$$
4 p+2 e^{-} \rightarrow^{4} \mathrm{He}+2 \nu_{e}+26.73 \mathrm{MeV} .
$$


Only $\sim 2 \%$ of the solar energy is emitted in the form of neutrinos, while the rest is radiated through photons.

In the three decades since the first experimental results on solar neutrinos, there has been a flourishing of solar models, all of which use the present values of some fundamental solar parameters (such as radius, mass, luminosity, $\mathrm{He} / \mathrm{H}$ ratio) as constraints on the stellar evolution. They all make essentially the same assumptions on the nature of the solar energy and of the solar energy transfer mechanism. The most famous and accredited model is certainly that of Bahcall-Pinsonneault (BP SSM).21 In the following we shall be referring to its two most recent versions, the BP9822 and BP2000 23 in terms of which most published experimental results are interpreted. All reactions belonging to the $p p$ and the CNO cycles, together with the corresponding average and maximum neutrino energies are listed in Tab.11(from ref.24). The CNO cycle contributes less than $2 \%$ to the total neutrino flux.

Table 1. Average and maximum mputrino energy for the different reactions contributing to the solar neutrino flux (tab. from ref 24 ).

\begin{tabular}{cccc}
\hline Source & Reaction & $<E_{\nu}>(\mathrm{MeV})$ & Max. $E_{\nu}(\mathrm{MeV})$ \\
\hline \hline & & & \\
$p p$ & $p+p \rightarrow d+e^{+}+\nu_{e}$ & 0.2668 & $0.423 \pm 0.03$ \\
$p e p$ & $p+e^{-}+p \rightarrow d+\nu_{e}$ & 1.445 & 1.445 \\
$h e p$ & ${ }^{3} \mathrm{He}+p \rightarrow 4^{\mathrm{He}}+e^{+}+\nu_{e}$ & 9.628 & 18.778 \\
${ }^{7} \mathrm{Be}$ & $e^{-}+{ }^{7} \mathrm{Be} \rightarrow{ }^{7} \mathrm{Li}+\nu_{e}$ & 0.3855 & 0.3855 \\
& & 0.8631 & 0.8631 \\
${ }^{8} \mathrm{~B}$ & ${ }^{8} \mathrm{~B} \rightarrow{ }^{8} \mathrm{Be}^{*}+e^{+}+\nu_{e}$ & $6.735 \pm 0.036$ & $\sim 15$ \\
${ }^{13} \mathrm{~N}$ & ${ }^{13} \mathrm{~N} \rightarrow{ }^{13} \mathrm{C}+e^{+}+\nu_{e}$ & 0.7063 & $1.1982 \pm 0.0003$ \\
${ }^{15} \mathrm{O}$ & ${ }^{15} \mathrm{O} \rightarrow{ }^{15} \mathrm{~N}+e^{+}+\nu_{e}$ & 0.9964 & $1.7317 \pm 0.0005$ \\
${ }^{17} \mathrm{~F}$ & ${ }^{17} \mathrm{~F} \rightarrow{ }^{17} \mathrm{O}+e^{+}+\nu_{e}$ & 0.9977 & $1.7364 \pm 0.0005$ \\
& & & \\
\hline
\end{tabular}

The neutrino fluxes predicted by BP2000, as well as the predicted neutrino capture rates in the chlorine and gallium experiments, are listed in Tab.2. The uncertainties on the neutrino absorption cross sections represent the dominant contribution to the error on the gallium rate predictions, while for the chlorine rate those uncertainties are relatively small compared to other systematic components intrinsic to the experimental technique. The rates are measured in Solar Neutrino Units (SNU), a convenient unit to describe the rates of solar neutrino experiments $\left(1 \mathrm{SNU}=10^{-36}\right.$ events atoms $\left.{ }^{-1} \mathrm{~s}^{-1}\right)$.

The energy spectra of neutrinos from the $p p$-cycle are shown in Fig.1 (BP2000 predictions, from ref.25). The uncertainties on the different flux components are also shown and the energy thresholds of the different experiments are indicated at the top of the plot.

There are several reasons, other than the high precision of the input data and calculations, why the BP SSM is believed to be robust. First of all, there is what is known as the "luminosity constraint" argument. It assumes that the Sun is in 


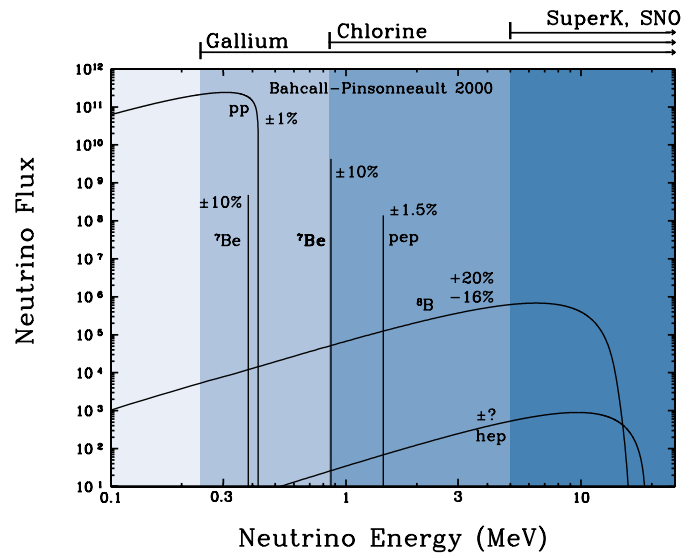

Fig. 1. BP200022 predictions for the energy spectra of solar neutrinos produced in the $p p$-c le reactions, which are responsible for more than $98 \%$ of the energy balance in the Sun (fig. from 25 ). The continuum spectra are expressed in events $\mathrm{cm}^{-2} \mathrm{~s}^{-1} \mathrm{MeV}^{-1}$ at one astronomical unit, while the monochromatic lines ( $p e p$ and ${ }^{7} \mathrm{Be}$ ) are given in events $\mathrm{cm}^{-2} \mathrm{~s}^{-1}$.

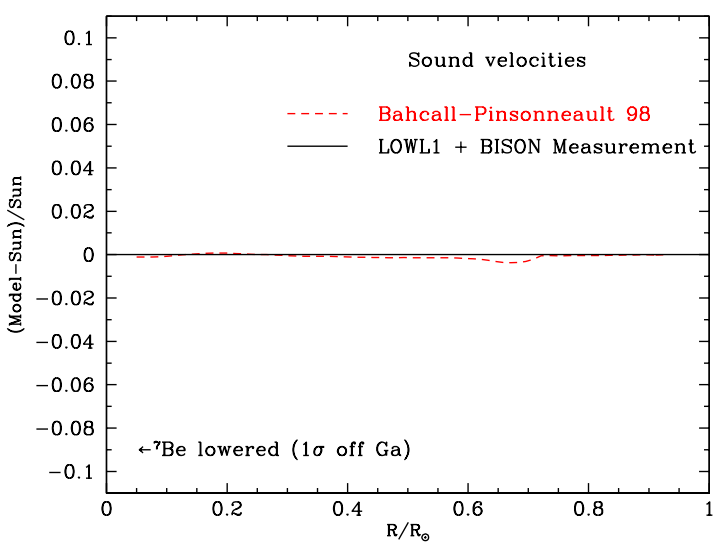

Fig. 2. Comparison between the predicted (Model) and measured (Sun) sound speeds in the Sun. Predictions come from the $\mathrm{BP} 98$ model 22 while measurements come from the helioseismological data on the sound speeds.26 
Table 2. BP200023 predictions for the solar neutrino fluxes and the neutrino capture rates in chlorine and gallium experiments.

\begin{tabular}{cccc}
\hline Source & Flux $\left(10^{10} \mathrm{~cm}^{-2} \mathrm{~s}^{-1}\right)$ & $\mathrm{Cl}(\mathrm{SNU})$ & $\mathrm{Ga}(\mathrm{SNU})$ \\
\hline \hline & & & \\
$p p$ & $5.95\left(1.00_{-0.01}^{+0.01}\right)$ & - & 69.7 \\
pep & $1.40 \times 10^{-2}\left(1.00_{-0.015}^{+0.015}\right)$ & 0.22 & 2.8 \\
hep & $9.3 \times 10^{-7}$ & 0.04 & 0.1 \\
${ }^{7} \mathrm{Be}$ & $4.77 \times 10^{-1}\left(1.00_{-0.10}^{+0.10}\right)$ & 1.15 & 34.2 \\
${ }^{8} \mathrm{~B}$ & $5.05 \times 10^{-4}\left(1.00_{-0.16}^{+0.20}\right)$ & 5.76 & 12.1 \\
${ }^{13} \mathrm{~N}$ & $5.48 \times 10^{-2}\left(1.00_{-0.17}^{+0.21}\right)$ & 0.09 & 3.4 \\
${ }^{15} \mathrm{O}$ & $4.80 \times 10^{-2}\left(1.00_{-0.19}^{+0.25}\right)$ & 0.33 & 5.5 \\
${ }^{17} \mathrm{~F}$ & $5.63 \times 10^{-4}\left(1.00_{-0.25}^{+0.25}\right)$ & - & 0.1 \\
\hline Total & & & \\
\hline
\end{tabular}

a stationary state and then infers a strong correlation between the solar neutrino flux and the solar luminosity $\left(\mathcal{L}_{\odot}=2.4 \times 10^{39} \mathrm{MeV} \mathrm{s}{ }^{-1}\right.$, known to an accuracy of $\sim 0.4 \%$ ). From Eq.(31) we see that, in the nuclear reactions occurring in the Sun, the production of two neutrinos is accompanied by an energy release of $26.73 \mathrm{MeV}$. Neglecting the small amount of energy carried away by the neutrinos, this means that the electron neutrino flux in the absence of oscillations can be estimated as

$$
\Phi_{\nu_{e}} \simeq 2 \times \frac{\mathcal{L}_{\odot} /\left(4 \pi R_{\odot}^{2}\right)}{26.73 \mathrm{MeV}}=6.4 \times 10^{10} \mathrm{~cm}^{-2} \mathrm{~s}^{-1},
$$

where $R_{\odot}$ is one astronomical unit $\left(=1.496 \times 10^{13} \mathrm{~cm}\right)$.

Another model-independent constraint, although looser than the luminosity one, is given by the fact that the helium nuclei, necessary for the boron and beryllium production, are created in the $p p$ and $p e p$ reactions. Thus:

$$
\Phi_{\nu_{e}}\left({ }^{7} \mathrm{Be}\right)+\Phi_{\nu_{e}}\left({ }^{8} \mathrm{~B}\right)<\Phi_{\nu_{e}}(p p)+\Phi_{\nu_{e}}(p e p) .
$$

One the most impressive checks of the BP SSM come perhaps from the comparison between the BP98 model predictions, obtained without any adjustment of the parameters, and the most accurate helioseismology measurements of the sound speed.26 Fig.2 shows the excellent agreement between the calculated and the measured values: the size of the fractional difference between measurements and predictions (0.001 standard deviation for radii between $5 \%$ and $95 \%$ of the Sun radius) is much smaller than any generic change in the model with an impact on the predicted neutrino flux. 


\subsection{Experimental Results}

We shall now describe the experimental techniques which have been used to detect solar neutrinos. The experiments can be subdivided into two categories: radiochemical experiments (Homestake, SAGE, GALLEX and its successor, the GNO27 project) and water-Cherenkov experiments (Kamiokande, Super-Kamiokande and $\mathrm{SNO}$ ). One of the main differences between these two categories is the fact that, while for radiochemical experiments only an integrated measurement of the fluxes is possible, water-Cherenkov detectors can perform real-time measurements and therefore can study differential distributions (i.e. energy spectrum, angular distribution, correlation with the Sun position in the sky).

The Homestake 15 . 16 chlorine experiment is located $1480 \mathrm{~m}$ underground (4200 m.w.e., meters water equivalent), at the Homestake gold mine, Lead, South Dakota, USA. A tank of $6 \times 10^{5}$ litres volume is filled with tetrachlorethylene $\left(\mathrm{C}_{2} \mathrm{Cl}_{4}\right)$. The detection principle is based on the reaction:

$$
\nu_{e}+{ }^{37} \mathrm{Cl} \rightarrow e^{-}+{ }^{37} \operatorname{Ar}\left(\mathrm{T}_{1 / 2}=35 \mathrm{~d}\right),
$$

which has a threshold of $814 \mathrm{keV}$, above the end-point of the $p p$-cycle energy spectrum. After an exposure time of $1-3$ months, the ${ }^{37} \mathrm{Ar}$ atoms produced in (34) are extracted by purging the detector with ${ }^{4} \mathrm{He}$ and detecting the Auger electron produced in the electron capture of the radioactive ${ }^{37} \mathrm{Ar}$ nuclei. The measured rate, which mainly arises from of ${ }^{8} \mathrm{~B}$ and ${ }^{7} \mathrm{Be}$ neutrinos, plus a small contribution from pep neutrinos, is $2.56 \pm 0.23 \mathrm{SNU} 16$ significantly below the predicted value of $7.6_{-1.1}^{+1.3}$ SNU. Although the Homestake detector is not calibrated, for lack of a suitable artificial neutrino source, the efficiency of the extraction technique has been checked by doping the detector with a known amount of radioactive argon atoms.

All gallium experiments, SAGE, 17 GALLEX 18 and GNO,27 detect neutrinos from the Sun by means of the process:

$$
\nu_{e}+{ }^{71} \mathrm{Ga} \rightarrow e^{-}+{ }^{71} \mathrm{Ge}\left(\mathrm{T}_{1 / 2}=11.43 \mathrm{~d}\right),
$$

for which the energy threshold $(233.2 \mathrm{keV})$ is well below the maximum energy of the $p p$ neutrinos. This means that gallium experiments, by measuring low-energy solar neutrinos, have the ability to prove the thermonuclear nature of the energy production mechanism in the Sun.

The GALLEX experiment, now discontinued as such, was located at the Gran Sasso Underground Laboratory (LNGS), Assergi, Italy. It consisted of 30.3 tons of

${ }^{71} \mathrm{Ga}$, in the form of a concentrated solution of gallium chloride $\left(\mathrm{GaCl}_{3}-\mathrm{HCl}\right)$ in water. The ${ }^{71} \mathrm{Ge}$ atoms form the volatile compound $\mathrm{GeCl}_{4}$ which, at the end of each run $(3-4$ weeks) is swept out of the solution by means of a nitrogen stream. The $\mathrm{GeCl}_{4}$ is absorbed in water in a gas scrubber, where the nitrogen is filtered out, and then converted to $\mathrm{GeH}_{4}$. This compound is finally introduced, together with xenon, into a proportional counter where the number of ${ }^{71} \mathrm{Ga}$ atoms is determined by counting the radioactive decays. The final results from GALLEX(I-IV) 18 give 
a solar neutrino flux of $77.5_{-7.8}^{+7.6} \mathrm{SNU}$, again well below theoretical predictions of $128_{-7}^{+9}$ SNU. The GALLEX detector was calibrated using two independent methods. The first one made use of neutrinos from two intense $(>60 \mathrm{PBq}){ }^{51} \mathrm{Cr}$ sources: the combined value of the ratio $R$ between the neutrino source strength as derived from the measured rate of ${ }^{71} \mathrm{Ga}$ production and the directly determined source strength is $R=0.93 \pm 0.08 .28$ The other calibration was performed by injecting under varying conditions, a known amount of ${ }^{71}$ As into the full-scale detector.29 The arsenic isotope decays by electron capture and positron emission to ${ }^{71} \mathrm{Ge}\left(\mathrm{T}_{1 / 2}=2.72 \mathrm{~d}\right)$, producing radioactive atoms which mimic the solar neutrino capture kinematics. Although neutrinos from the ${ }^{51} \mathrm{Cr}$ source provide a better match to solar neutrino energies, the second method has the great advantage of large statistics. The measured recovery rate of ${ }^{71} \mathrm{Ge}$ from gallium is $1 \pm 0.01$. The two results 2829 together rule out the possibility that the solar neutrino deficit observed by GALLEX can be attributed to systematic errors in the radiochemical extraction procedure.

The SAGE17 experiment is carried out by a Russian-American collaboration at the underground Baksan Neutrino Observatory (4715 m.w.e.), in the Northern Caucasus mountains. The detector, which weighs 57 tons, uses gallium in its metallic form. The germanium produced in (35) is removed from the metallic gallium by a liquid-liquid extraction into a $\mathrm{HCl}-\mathrm{H}_{2} \mathrm{O}_{2}$ phase. After this initial step, the experimental technique is very similar to that followed by GALLEX. The results reported by the SAGE collaboration are based on a 10 years exposure time (from January 1990 to October 1999), yielding a measured solar neutrino flux of $74_{-7.4}^{+7.8}$ (stat.+sys.) SNU 17 in very good agreement with the GALLEX results. The SAGE detector has been calibrated, exposing 13 tons of metallic gallium to an intense ${ }^{51} \mathrm{Cr}$ source. 30 As for GALLEX, the ratio of the measured production rate to that predicted from the source activity, $R=0.95 \pm 0.11$ (stat. $)_{-0.08}^{+0.05}$, has confirmed that the extraction procedure is reliable.

In 1998, after an upgrade of the existing experimental setup, the GNO (Gallium Neutrino Observatory) experiment27 started operation at LNGS, as the successor project to the GALLEX experiment. The main aims of GNO are to provide a long time record of low-energy solar neutrinos, to determine the bulk production rate with an accuracy of $5 \mathrm{SNU}$ and to monitor the time dependence of the $p p$-neutrino flux during a whole solar cycle to a precision of $\sim 15 \%$. The GNO collaboration has recently reported the results from the GNO I phase of the experiment, based on 19 months of observation (from May 1998 to January 2000). The measured solar neutrino flux is $65.8_{-10.2}^{+10.7}$ (stat.+sys.) SNU 27 which confirms the GALLEX result. A combined analysis of GNO I and GALLEX gives $74.1_{-6.8}^{+6.7} \mathrm{SNU} .27$

Both Kamiokande 19 and Super-Kamiokande 20 are water-Cherenkov detectors, located $1000 \mathrm{~m}$ underground (2700 m.w.e.), in the Kamioka mine in Japan.

Kamiokande started its operation in 1984 and was originally intended to study nucleon decay. 31 It was later upgraded to detect also low energy events and succeeded in observing the first solar neutrinos in 1987. Kamiokande was made of a cylindrical tank, of total volume $4.5 \mathrm{kton}$, filled with pure water. An inner volume of 
2.14 kton was defined by 980 inward-looking photo-multiplier tubes (PMTs), used to detect the Cherenkov light produced by the relativistic particles traversing the water. Kamiokande ended physics data-taking at the beginning of 1995 and was completely stopped in summer 1997. Since then its physics programme has been continued by its successor experiment, Super-Kamiokande.

Super-Kamiokande consists of a huge cylindrical tank (volume 50 kton) filled with pure water (water transparency is $\sim 100 \mathrm{~m}$ at $\lambda=420 \mathrm{~nm}$ ). Also in this case an inner volume (16.9 m diameter, $36.2 \mathrm{~m}$ height, volume $32.5 \mathrm{kton}$ ) is defined by an inner surface equipped with a large number of PMTs $(11,146$ PMTs of $50 \mathrm{~cm}$ diameter, $40 \%$ surface coverage). The increased coverage of PMTs makes SuperKamiokande not only bigger than Kamiokande, but also better in terms of energy, position and angular resolution. An outer volume, $2 \mathrm{~m}$ thick, equipped with 1,185 PMTs (20 cm diameter) surrounds the inner detector and serves as an active veto counter against gamma-rays, neutrons and through-going cosmic muons. For the solar neutrino analysis, the fiducial mass is $22.5 \mathrm{kton}$, the fiducial volume boundaries being $2 \mathrm{~m}$ inside the inner surface.

In both Kamiokande and Super-Kamiokande the solar neutrinos are detected through the observation of the Cherenkov rings produced by the electrons emitted in the elastic process:

$$
\nu_{x}+e^{-} \rightarrow \nu_{x}+e^{-} \quad(x=e, \mu, \tau) .
$$

Although the cross-sections for this process are very small, ${ }^{f}$ elastic interactions turn out to be very useful, thanks to the nice correlation between the recoil electron momentum direction and the direction of the incoming neutrino $\left(E_{e} \theta_{r e c} \leq 2 m_{e}\right.$, $\theta \sim 18^{0}$ for $10 \mathrm{MeV}$ neutrinos). Therefore the direction of the recoil electron can be used to correlate the direction of the impinging neutrino with the Sun's position in the sky (see Fig.33). Moreover the energy of the recoiling electron can be used to obtain a lower limit on the incoming neutrino energy.

The total released energy is correlated to the number of PMT hits, which is in turn a function of the total emitted light, corrected for light absorption through water, PMT geometry and overlapping hits. The energy scale, however, must be determined in an independent manner.

Kamiokande used gamma-rays from the $\mathrm{Ni}(\mathrm{n}, \gamma) \mathrm{Ni}$ reaction to obtain it. 1 The measurement precision was at the $1-2 \%$ level, the main limitation being the knowledge of the branching ratios and of the neutron absorption cross-sections for different nickel isotopes. Only limited information could be extracted about the energy resolution, while no information at all could be obtained on the angular resolution of the detector.

Super-Kamiokande determines the neutrino energy scale by injecting, at various positions of the inner detectors, electrons from an electron LINAC placed near the

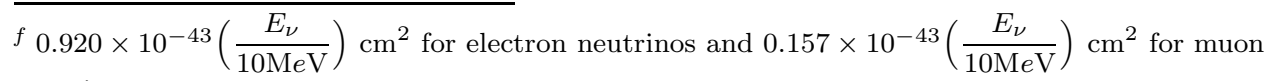
neutrinos. 


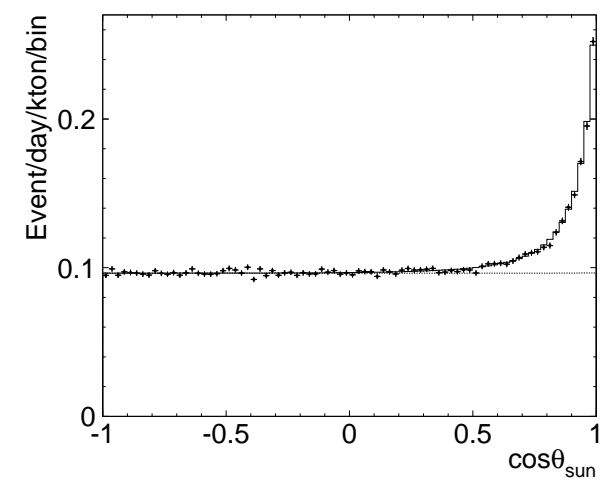

Fig. 3. Super-Kamiokande (1258 days) distribution of the cosine ff the angle $\theta_{\text {sun }}$ between the recoil electron momentum and the vector from the Sun to the Earth 32 The dots are the experimental data, while the solid histogram is the best fit for signal plus background. The peak emerging at $\cos \theta_{\text {sun }}$ over a flat background is due to solar neutrinos.

detector tank 33 Electrons from the LINAC span the energy range $5-16 \mathrm{MeV}$, which matches exactly the region relevant for solar neutrinos. By means of this procedure, the absolute energy scale is known to better than 1\%. Moreover, Monte Carlo studies show that the energy resolution of the detector can be reproduced to an accuracy of $2 \%$, while the angular resolution is reproduced to better than $1.5^{0}$ for $10 \mathrm{MeV}$ electrons.

In order to check these results and, what is possibly even more important, in order to reduce the intrinsic systematic error inherent in the described procedure, ${ }^{g}$ another calibration technique has been introduced 34 A pulsed deuterium-tritium neutron generator produces isotropically distributed neutrons of $14.2 \mathrm{MeV}$, via the reaction ${ }^{3} \mathrm{H}+{ }^{2} \mathrm{H} \rightarrow{ }^{4} \mathrm{He}+n$. The neutrons create ${ }^{16} \mathrm{~N}$ by the $(n, p)$ reaction on ${ }^{16} \mathrm{O}$ in the water. ${ }^{16} \mathrm{~N}$ decays $(\tau=7.13 \mathrm{~s}, Q=10.4 \mathrm{MeV})$ are characterised by the emission of a $4.3 \mathrm{MeV}$ electron in coincidence with a $6.1 \mathrm{MeV}$ gamma-ray and they are therefore well suited to verify the solar neutrino absolute energy scale. Additional ${ }^{16} \mathrm{~N}$ is also naturally produced in cosmic muon capture by ${ }^{16} \mathrm{O}$. These events, which are selected out of the full data sample and which are generally used to monitor the solar neutrino signal extraction method, can be also analysed to check the absolute energy scale. Excellent agreement (within 1\%) has been found between the data and the MC, previously tuned on the LINAC data. Moreover it has been possible to show that the position and the angular dependence of the energy scale are within systematic uncertainties for the energy scale of the detector.

The energy threshold for Kamiokande and Super-Kamiokande is determined by

\footnotetext{
$g$ electrons from the LINAC only move in a downward direction; due to limited access, the beam pipe and the LINAC calibration equipment can only be operated at a finite number of positions; the presence of the beam pipe in the tank limits the calibration procedure precision and is in fact the main source of systematic uncertainties at low energy.
} 
the threshold for detecting the recoil electron in the elastic scattering (36), which is about $5-7.5 \mathrm{MeV}$. Thus the solar neutrino flux measured in those experiments is essentially the ${ }^{8} \mathrm{~B}$ flux (plus a small component from the hep reaction in SuperKamiokande). The value measured by Kamiokande, based on 2079 days of datataking (from January 1987 to February 1995), is $2.80 \pm 0.19$ (stat.) \pm 0.33 (sys.) $\times$ $10^{6} \mathrm{~cm}^{-2} \mathrm{~s}^{-1} 19$ The Super-Kamiokande collaboration has recently reported a result based on 1258 days of data-taking, for recoil electrons in the energy range $5-$ $20 \mathrm{MeV}$. The measured neutrino flux is $2.32 \pm 0.03$ (stat.) ${ }_{-0.07}^{+0.08}$ (sys.) $\times 10^{6} \mathrm{~cm}^{-2} \mathrm{~s}^{-1} 32$ For the hep neutrinos they set an upper limit of $4 \times 10^{4} \mathrm{~cm}^{-2} \mathrm{~s}^{-1}$, at $90 \%$ C.L.

Before we move to other issues relevant for the understanding solar neutrino puzzle, we summarize the results discussed up to now and their implications on neutrino physics. In Tab.3 the measured values of the integrated solar neutrino fluxes are compared with the BP2000 model predictions 23.

Table 3. Compariso etween the measured integrated solar neutrino fluxes and the corresponding BP2000 predictions 23 Units are SNU for chlorine and gallium experiments and $10^{6} \mathrm{~cm}^{-2} \mathrm{~s}^{-1}$ for ${ }^{8} \mathrm{~B}$ and hep neutrinos.

\begin{tabular}{|c|c|c|c|}
\hline Experiment & Measured & BP2000 & Measured/BP2000 \\
\hline Chlorine 16 & $2.56 \pm 0.23$ & $7.6_{-1.1}^{+1.3}$ & $0.34 \times(1.00 \pm 0.06)$ \\
\hline GALLEX+GNOL27 & $74.1_{-7.8}^{+6.7}$ & $128_{-7}^{+9}$ & $0.58 \times\left(1.00_{-0.12}^{+0.11}\right)$ \\
\hline SAGE & $75.4_{-7.4}^{+7.8}$ & $128_{-7}^{+9}$ & $0.59 \times(1.00 \pm 0.12)$ \\
\hline${ }^{8}$ B-Kamiokande 19 & $2.80 \times(1.00 \pm 0.14)$ & $5.05 \times\left(1.00_{-0.16}^{+0.20}\right)$ & $0.55 \times\left(1.00_{-0.21}^{+0.24}\right)$ \\
\hline${ }^{8}$ B-Super-Kamiokande 32 & $2.32 \times(1.00 \pm 0.03)$ & $5.05 \times\left(1.00_{-0.16}^{+0.20}\right)$ & $0.46 \times\left(1.00_{-0.16}^{+0.20}\right)$ \\
\hline hep-Super-Kamiokande 22 & $<40 \times 10^{-3}$ & $9.3 \times 10^{-3}$ & $<4.3$ \\
\hline
\end{tabular}

In brief, all experiments see a deficit of solar neutrinos compared to predictions. We have seen that the solar model's predictions appear to be robust, and so are the experimental results (although the Homestake experiment is sometimes still being questioned because of the lack of a calibration). Therefore neither an astrophysical solution to the solar neutrino puzzle, nor attributing the discrepancy between theory and experiments to not completely understood systematic uncertainties seem acceptable. There must be a particle physics solution to the solar neutrino deficit, which is however more than a sole normalisation problem. The impossibility of accommodating all the experimental results in a consistent picture forces us to go beyond the standard physics framework and to look for explanations which involve new phenomenology.

The internal inconsistency of the solar neutrino results is very nicely summarised 


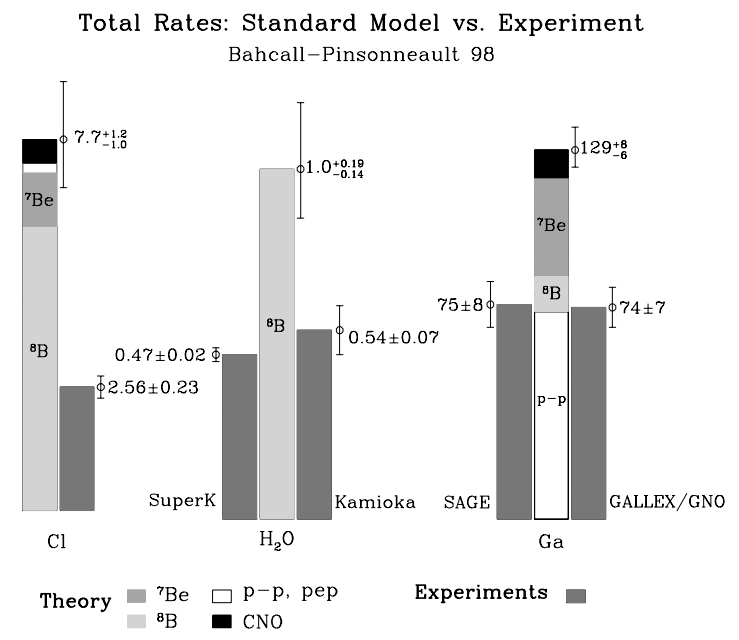

Fig. 4. Measured vs. predicted solar neutrino rates (fig. from ref.25) for all the solar neutrino experiments. The predigted rates for the various components of the neutrino spectrum are taken from the BP98 model.22

in Fig. (from ref.25), where a breakdown of all the measured and calculated event rates is shown as a function of the experimental technique and of the different reactions contributing to the solar neutrino flux. First of all there is the low rate observed in the chlorine experiment, which basically measures ${ }^{8} \mathrm{~B}$ neutrinos, with smaller contributions from ${ }^{7} \mathrm{Be}$ and $\mathrm{CNO}$ neutrinos. Second, there is the incompatibility between the chlorine and the water-Cherenkov experiments. In fact, since the shape of the ${ }^{8} \mathrm{~B}$ neutrino energy spectrum is very stable under reasonable changes of the solar parameters, in the absence of new physics it should be possible to compute the ${ }^{8} \mathrm{~B}$ rate in $\mathrm{Cl}$ from the spectrum observed by Kamiokande and SuperKamiokande, which essentially measure the ${ }^{8} \mathrm{~B}$ flux at high energies. But the number obtained from the analysis of the water-Cherenkov data is by itself higher than the total flux measured by Homestake. This result, taken as such, implies that the net contribution of the pep, ${ }^{7} \mathrm{Be}$ and $\mathrm{CNO}$ reactions to the total chlorine experiment flux is negative. In other words, the Homestake measurement is compatible with the ${ }^{7}$ Be neutrinos being completely suppressed, and this is indeed a big puzzle, since beryllium is needed for the production of ${ }^{8} \mathrm{~B}$ neutrinos. Finally, examining the gallium experiments result in detail, one finds that essentially all of the measured flux can be accounted for by only considering the contribution from the $p p$-cycle, which is known within $1 \%$ and therefore is not questionable. Ignoring for simplicity the small contribution from ${ }^{8} \mathrm{~B}$ neutrinos (which, by the way, can also be derived from water-Cherenkov results), this means that there is no room to accommodate the significant contribution expected from the ${ }^{7} \mathrm{Be}$ lines. 
The strong correlation between the predicted ${ }^{7} \mathrm{Be}$ and the ${ }^{8} \mathrm{~B}$ fluxes is shown in Fig. 5 for many different solar models, together with the best fit solution for the ${ }^{7} \mathrm{Be}$ flux as obtained from experimental data. All the fluxes have been normalised to the predictions of the BP98 model and a $3 \sigma$ rectangle has been drawn, showing that all models but one (that of Dar-Shavive 36 ) agree with each other within $3 \sigma$ of the BP98 model and are all far away from the value derived from experimental data.

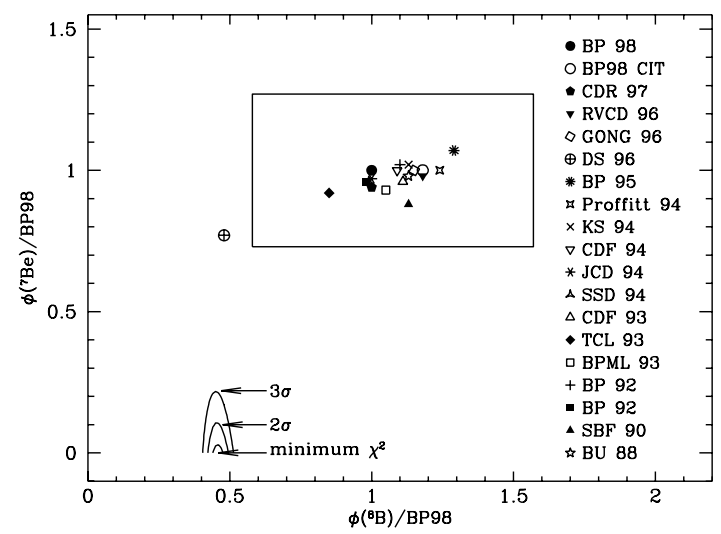

Fig. 5. Correlation between the ${ }^{7} \mathrm{Be}$ and the ${ }^{8} \mathrm{~B}$ fluxes (normalised to BP98 predictions) as given by published solar models (fig. from ref.35). The rectangle corresponds to the $3 \sigma$ contour for the BP98 model prediction and the ellipses are the 1,2,3 $\sigma$ contours for the best fit solution as obtained from all experimental data assuming no new physics.

In summary, if one believes the conclusions of the standard solar models, the solar neutrino puzzle can only be solved if one is either ready to discard at least three experimental results (the chlorine experiment and either the gallium or the water-Cherenkov measurements), which seems unreasonable, or to accept that new physics, not accounted for in the standard model of electro-weak interactions, is modifying the energy spectrum of solar neutrinos.

One very attractive possibility is that neutrino oscillations are changing the neutrino flavour composition while they travel between the centre of the Sun and the Earth. If solar electron neutrinos have transformed into muon or tau neutrinos before reaching the detector, given their low average energy they would fail to produce muons or taus via charged-current interactions. A "disappearance" of electron neutrinos in the chlorine and gallium experiments would then become an expected effect. The deficit in the water-Cherenkov experiments, where in principle muon and tau neutrinos could be detected via their neutral-current scatters with electrons of the medium, could also be explained by the low cross-section for that process.

Interpreting the solar neutrino deficit in the two-flavour mixing framework, allowed regions can be drawn on the oscillation parameter space $\left\{\sin ^{2} 2 \theta, \Delta m^{2}\right\}$ at a 

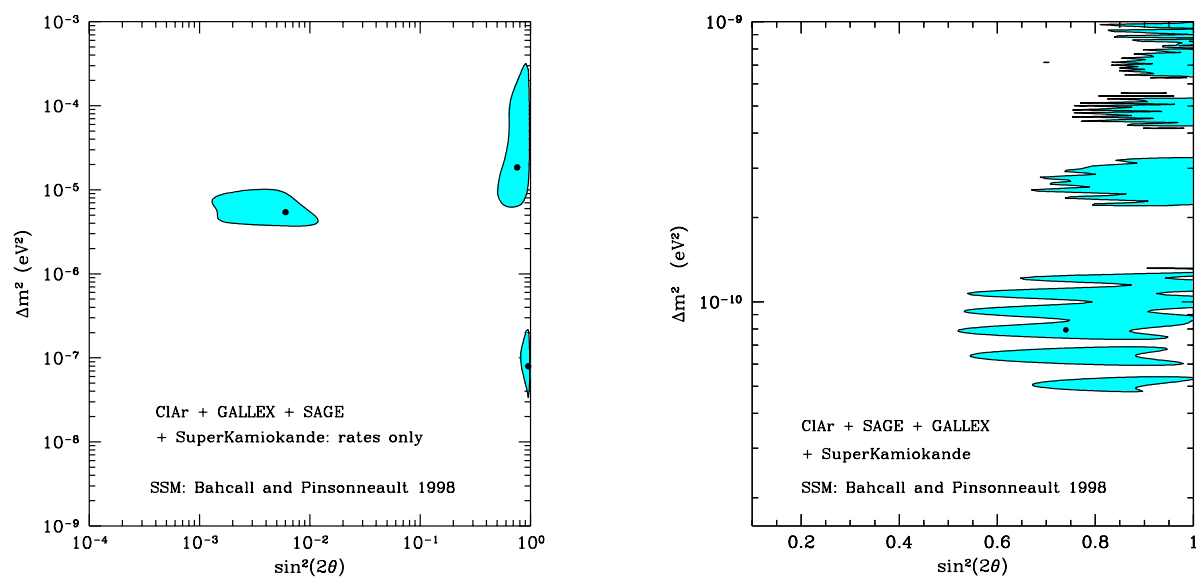

Fig. 6. Allowed regions (99\% C.L.) on the plane $\left(\sin ^{2} 2 \theta, \Delta m^{2}\right)$, as obtained in ref. 55 from a combined two-flavour analysis of the total rate results in the chlorine, SAGE, GALLEX and Super-Kamiokande experiments, for oscillations in matter (left) and in vacuum (right).

set C.L.. ${ }^{h}$ For example, Fig.6 shows the 99 \% C.L. solutions as obtained in ref 3 s from a combined analysis of the total rate results in the chlorine, SAGE, GALLEX and Super-Kamiokande experiments, for oscillations in matter (MSW solutions, left) and in vacuum ("Just-so" or VO solutions, right). The matter solutions are conventionally named according to the allowed ranges of the oscillation parameters: small mixing angle (SMA, $\Delta m^{2} \sim 10^{-5} \mathrm{eV}^{2}, \sin ^{2} 2 \theta \sim 10^{-3}-10^{-2}$ ), large mixing angle (LMA, $\Delta m^{2} \sim 10^{-5}-10^{-4} \mathrm{eV}^{2}, \sin ^{2} 2 \theta>0.5$ ) and low $\Delta m^{2}$ (LOW, $\left.\Delta m^{2} \sim 10^{-7} e \mathrm{~V}^{2}, \sin ^{2} 2 \theta>0.9\right)$. The VO solution $\left(\Delta m^{2}<10^{-9} e \mathrm{~V}^{2}\right)$ corresponds to the case when one astronomical unit exactly matches the neutrino oscillation length for typical solar energies.

One way to discriminate between small and large mixing angles is to consider the so-called "day/night effect". Neutrinos detected during night, as opposed to neutrinos detected during day, are subject to matter effects in the Earth, which are expected to be sizeable for large values of the mixing angle. The solar neutrino flux can be studied as a function of the solar zenith angle $\theta_{z}$ and an asymmetry $\mathcal{A}$ can be defined:

$$
\mathcal{A}=\frac{\Phi_{N}-\Phi_{D}}{\Phi_{N}+\Phi_{D}}
$$

where $\Phi_{N}$ and $\Phi_{D}$ are the night and day fluxes respectively. A non-zero asymmetry

${ }^{h}$ As stressed in ref.37, under certain conditions the MSW solutions to the solar neutrino deficit can extend to the $\pi / 4<\theta<\pi / 2$ domain, which is not covered by the standard two-flavour analyses; to overcome this problem, results can be more conveniently presented in the $\left\{\tan ^{2} \theta, \Delta m^{2}\right\}$ parameter space. 
would favour large mixing angles. Another way to separate between the two regimes is to consider the shape of the solar zenith angle distribution, which is expected to be flat for large mixing angles, while for small mixing angles it should show an excess of events in the upward-going direction (around $-1<\cos \theta_{z}<-0.8$ ).
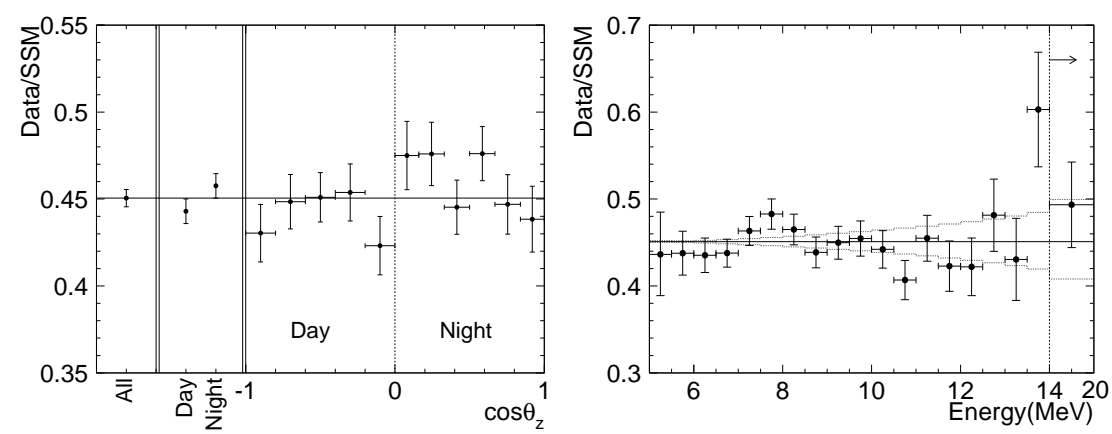

Fig. 7. Super-Kamiokande (1258 darg) measured distributions for the solar zenith angle (left) and of the solar neutrino energy (right). 22

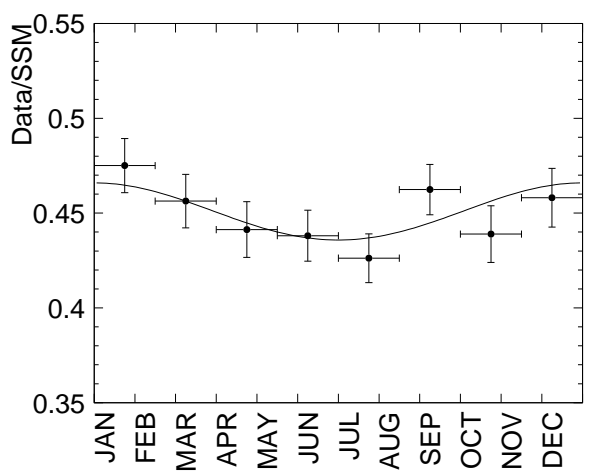

Fig. 8. Super-Kamiokande (1258 days) measurement of the seasonal variatim of the solar neutrino flux, not corrected for the effect due to the orbit eccentricity of the Earth.32

Oscillations would also manifest themselves as a distortion of the neutrino energy spectrum and it is therefore important to study the neutrino energy distribution, which for Super-Kamiokande is approximated by the recoil electron energy. It turns out that, if the predicted hep neutrino flux is correct, a distribution peaked at high energies would favour the VO solution.

Finally, strong evidence for neutrino oscillations in vacuum would come from the observation of a seasonal variation of the solar neutrino flux, different from the standard geometrical one due to the orbit eccentricity of the $\operatorname{Earth}(\epsilon=0.0167){ }^{i}$

\footnotetext{
${ }^{i}$ For $\Delta m^{2}$ of the order of that suggested by the VO solution, and for solar neutrino energies of the order of $\sim$ few $\mathrm{MeV}$, the seasonal change in the distance between the Sun and the Earth $\left(\Delta R_{\odot}=\right.$
} 
The day-night asymmetry obtained by Super-Kamiokande based on 1258 days of data is $\mathcal{A}=0.033 \pm 0.022$ (stat.) $)_{-0.012}^{+0.013}$ (sys.), 32 which is only a $1.3 \sigma$ deviation from zero asymmetry. The zenith angle distribution (Fig.7), left) is rather flat and shows no feature which might lead to a preference for the SMA solution. The measured energy spectrum shows no statistically significant distortion relative to the predicted ${ }^{8} \mathrm{~B}$ spectrum (Fig.7, right). No deviation from the expected standard annual variation of the neutrino flux has been observed (see Fig.8).

The Super-Kamiokande collaboration has recently performed a flux-independent $\chi^{2}$ analysis of their data, assuming two-flavour mixing. The resulting exclusion plot (95\% C.L.) for the $\nu_{e} \rightarrow \nu_{\mu, \tau}$ case is shown in Fig.9 (shaded areas), together with the allowed areas obtained using the zenith angle distribution and the SSM predictions (dotted) and the allowed areas obtained from a combined analysis of the GALLEX, SAGE, Homestake and Super-Kamiokande flux measurements (hatched). From this analysis the SMA and the VO solutions seem to be disfavoured at 95\% C.L. The same analysis repeated for pure $\nu_{e} \rightarrow \nu_{s}$ shows that all solutions are disfavoured at $95 \%$ C.L. for the sterile neutrino hypothesis.

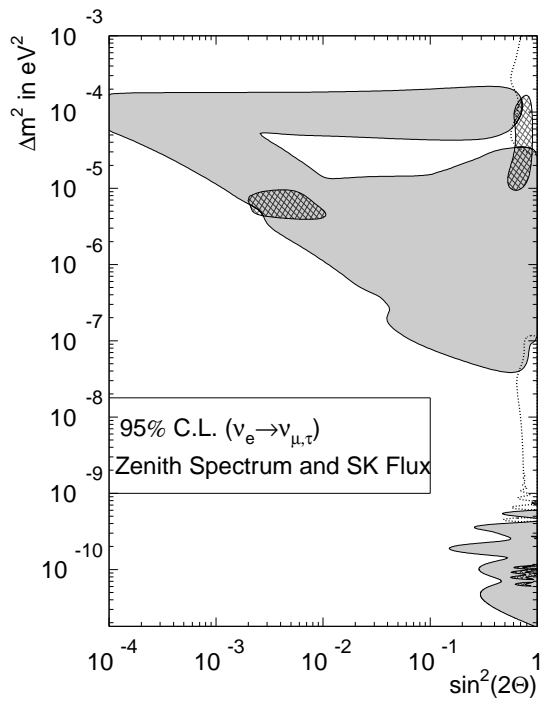

Fig. 9. Super Kamiokande exclusion areas for solar neutrino oscillations (shaded area, based on 1258 days). 32 The allowed areas obtained using the zenith angle distribution and the SSM predictions (dotted line) and those obtained combining all the experimental results on integrated solar neutrino fluxes (hatched islands) are also shown.

Although Super-Kamiokande has measured the energy dependence and the zenith angle dependence of the solar ${ }^{8} \mathrm{~B}$ flux, and extracted useful information about the

$2 \epsilon R_{\odot}$ ) is a sizeable fraction of the oscillation length in vacuum, $\lambda=4 \pi E / \Delta m^{2}$ (numerically, $\left.2 \times 0.016 \times\left(1.496 \times 10^{11} \mathrm{~m}\right) \times\left(2 \times 10^{-10} \mathrm{eV}^{2}\right) / 1 \mathrm{MeV} \sim 1\right)$. 
MSW and the VO solutions out of an impressively high-quality and high-statistics sample, the solar neutrino problem can by no means be considered definitively solved. Many questions still remain open and it is therefore fair to say that still lot of information is missing and further experimental effort is required. Along this line there come the Sudbury Neutrino Observatory project (SNO), 38 which has been running for about one year, as well as the Borexind 39 and the KamLAND 40 experiments, which are both scheduled to start data taking very soon.

$\mathrm{SNO}$ is a 1 kton heavy-water Cherenkov detector located $2 \mathrm{~km}$ underground (6010 m.w.e), in the Creighton mine, Sudbury, Ontario, Canada. The detector is made of a spherical acrylic vessel, $12 \mathrm{~m}$ diameter, containing ultra-pure $\mathrm{D}_{2} \mathrm{O}$ and surrounded by an ultra-pure $\mathrm{H}_{2} \mathrm{O}$ shield, in turn contained in a cylindrical cavity (34 m height, $22 \mathrm{~m}$ maximum diameter). The light produced in the water is detected by 9,456 PMTs (20 cm diameter) with light concentrator, installed on a stainless steel structure surrounding the acrylic vessel.

As well as using the elastic scattering (ES) reaction (36), as Kamiokande and Super-Kamiokande do, $\mathrm{SNO}$ can detect ${ }^{8} \mathrm{~B}$ solar neutrinos via charged-current $(\mathrm{CC})$ and neutral-current $(\mathrm{NC})$ interactions on deuterium:

$$
\nu_{e}+d \rightarrow p+p+e^{-} \quad(\text { threshold }=1.4 \mathrm{MeV})
$$

and:

$$
\nu_{x}+d \rightarrow n+p+\nu_{x} \quad(\text { threshold }=2.2 \mathrm{MeV}) .
$$

For completeness, we give again the ES reaction (36):

$$
\nu_{x}+e^{-} \rightarrow \nu_{x}+e^{-} \quad(x=e, \mu, \tau) .
$$

Neutral current interactions do not depend on the flavour of the incident neutrino and therefore neutrino oscillations to active neutrinos should not affect the number of $\mathrm{NC}$ events. On the other hand, given the energy range for solar neutrinos, $\nu_{\mu}$ and $\nu_{\tau}$ coming from oscillated $\nu_{e}$ would be below threshold for the production of their charged partners: the CC sample would then be depleted by neutrino oscillations. This means that the $\mathrm{CC} / \mathrm{NC}$ ratio can be used as a powerful probe to test the oscillation hypothesis. Moreover, following the same argument, any distortion in the measured $\mathrm{CC}$ energy spectrum with respect to predictions would be another signature of new physics.

The electron produced in (38), as opposed to that produced in (36), carries most of the neutrino kinetic energy in the final state. Therefore it provides a better estimate of the initial neutrino energy (resolution of $\sim 20 \%$ for the range of interest) and can be used to observe possible distortions of the neutrino energy spectrum.

While electrons produced in CC and ES reactions are detected via the associated Cherenkov light, neutrons from reaction (39) are detected using different techniques during the three different phases of the experiment. In the first phase, recently concluded, when only heavy water was used, they were measured through 
the $6.25 \mathrm{MeV}$ photons produced in the neutron capture (25\% efficiency); in the current phase, when 2.5 tons of $\mathrm{NaCl}$ have been added to the water, they are seen through the $8.6 \mathrm{MeV}$ photons produced in the neutron capture on $\mathrm{Cl}$ (85\% efficiency); finally, and this will be SNO's third phase, the salt will be removed and ${ }^{3} \mathrm{He}$ proportional counters will be installed, allowing direct detection of the neutrons ( $45 \%$ efficiency) and measurement of the spectra with completely different systematics.

The sample collected during the first phase, when the sensitivity to NC was lower, is essentially dominated by CC interactions, with a smaller component from ES events. Therefore the $\mathrm{CC} / \mathrm{NC}$ comparison will not be possible until data from the second phase, with the NC rate highly enhanced by the addition of salt, become available. However some information on neutrino oscillations can already be extracted from the first phase data, by comparing the CC and ES rates. In fact, while the CC reaction (38) is only sensitive to $\nu_{e}$ 's, because of the low solar neutrino energy, the ES reaction (36) is sensitive to all active neutrinos, although with a reduced sensitivity to $\nu_{\mu}$ and $\nu_{\tau}$. Thus, if neutrino oscillations change the flavour composition of the solar neutrino flux, transforming a fraction of the original $\nu_{e}$ 's into other active neutrino species, the CC sample will be depleted, while the ES sample remains unchanged. In other words, if the CC and ES measurements of the ${ }^{8} \mathrm{~B}$ electron neutrino fluxes give:

$$
\Phi_{8_{\mathrm{B}}}^{C C}\left(\nu_{e}\right)<\Phi_{8_{\mathrm{B}}}^{E S}\left(\nu_{e}\right),
$$

then, without having to refer to any particular solar model, the inequality between the two measured values can by itself be interpreted as an indication of neutrino oscillations to active neutrinos.

The SNO collaboration has very recently published their first results on ES and CC reactions, based on 240.95 live days of data. 3 In order to avoid biases in the event selection procedure, the data has been sub-divided in two subsets, with $70 \%$ of them used to establish the analysis procedure and the remaining $30 \%$ used as a blind sample to validate the analysis itself. The final data set consists of 1169 events.

The different contributions from CC, ES and residual neutron events are extracted by means of a maximum likelihood function, which combines the information from three variables: the effective kinetic energy of the event, the angle between the reconstructed direction of the event and the instantaneous Sun-to-Earth direction and a volume-weighted radial variable, related to the position of the event inside the detector. The breakdown of the events as obtained from the likelihood maximisation is given in Tab.4.

SNO data show that the shape of the CC energy spectrum is consistent with the Bahcall SSM predictions within one standard deviation. However, the low ratio of the measured ${ }^{8} \mathrm{~B}$ flux to SSM predictions $230.347 \pm 0.029$ confirms the solar neutrino deficit. Moreover it is smaller than what obtained by Super-Kamiokande from the analysis of ES events. 32 
Table 4. Contribution from CC, ES and neutron evepts to the neutrino interaction sample extracted from 240.95 live days of SNO data, first phase 3 The quoted errors are statistical only.

\begin{tabular}{cc}
\hline Sample & Nr. of events \\
\hline CC & $975.4 \pm 39.7$ \\
NC & $106.1 \pm 15.2$ \\
neutron & $87.5 \pm 24.7$ \\
\hline
\end{tabular}

Using events with kinetic energy above $6.75 \mathrm{MeV}$, the ${ }^{8} \mathrm{~B}$ fluxes as measured from CC and ES interactions in SNO are:

$$
\begin{aligned}
& \left.\Phi_{S N O}^{C C}\left(\nu_{e}\right)=\left(1.75 \pm 0.07(\text { stat. })_{-0.11}^{+0.12}(\text { sys. }) \pm 0.05 \text { (theor. }\right)\right) \times 10^{6} \mathrm{~cm}^{-2} \mathrm{~s}^{-1} \\
& \Phi_{S N O}^{E S}\left(\nu_{x}\right)=\left(2.39 \pm 0.34(\text { stat. })_{-0.14}^{+0.16}(\text { sys. })\right) \times 10^{6} \mathrm{~cm}^{-2} \mathrm{~s}^{-1}
\end{aligned}
$$

where the theoretical uncertainty in (41) is that on the total CC cross section.

The difference between $\Phi_{S N O}^{C C}\left(\nu_{e}\right)$ and $\Phi_{S N O}^{E S}\left(\nu_{e}\right)$ is $(0.64 \pm 0.40) \times 10^{6} \mathrm{~cm}^{-2} \mathrm{~s}^{-1}$, that is to say a discrepancy of only $1.6 \sigma$. However the SNO measurement of the ES flux is consistent with that measured with higher precision by Super-Kamiokande: 22

$$
\Phi_{S K}^{E S}\left(\nu_{x}\right)=\left(2.32 \pm 0.03(\text { stat. })_{-0.07}^{+0.08}(\text { sys. })\right) \times 10^{6} \mathrm{~cm}^{-2} \mathrm{~S}^{-1},
$$

which permits to make a direct comparison between $\Phi_{S N O}^{C C}\left(\nu_{e}\right)$ and $\Phi_{S K}^{E S}\left(\nu_{x}\right)$.

The difference between the ES flux measured by Super-Kamiokande and the CC flux measured by SNO is $(0.57 \pm 0.17) \times 10^{6} \mathrm{~cm}^{-2} \mathrm{~s}^{-1}$, this time corresponding to a more significant $3.3 \sigma$ discrepancy. This result, by showing that the electron neutrino flux from CC interactions is indeed smaller than that obtained from ES events, provides quite a convincing hint for appearance of non-electron active neutrino flavours in the solar neutrino flux.

If electron neutrinos oscillated to sterile neutrinos, the ${ }^{8} \mathrm{~B}$ flux derived by SNO using $\mathrm{CC}$ events with energy above $6.75 \mathrm{MeV}$ should be consistent with the flux measured by Super-Kamiokande using ES interactions above $8.5 \mathrm{MeV}$. 11 Instead, correcting for the different energy threshold used in the latest Super-Kamiokande analysis, 32 the two fluxes differ by $(0.53 \pm 0.17) \times 10^{6} \mathrm{~cm}^{-2} \mathrm{~s}^{-1}$, that is to say $3.1 \sigma$. Therefore the SNO data exclude the possibility that the solar neutrino deficit can be explained by pure $\nu_{e} \rightarrow \nu_{s}$ oscillations.

In the hypothesis that the observed solar neutrino deficit is due to oscillations of $\nu_{e}$ 's to other active neutrino species $\left(\nu_{\mu}\right.$ and $\left.\nu_{\tau}\right)$, the flux of non-electron active neutrinos, $\Phi_{\mu \tau}$, can be inferred. The obtained correlation between $\Phi_{\mu \tau}$ and $\Phi_{e}$ is shown in Fig 10. The best fit corresponds to a non-electron active neutrino flux of:

$$
\Phi_{\mu \tau}=(3.69 \pm 1.13) \times 10^{6} \times \mathrm{cm}^{-2} \mathrm{~s}^{-1} .
$$

The two diagonal bands correspond to constant total flux of active ${ }^{8} \mathrm{~B}$ neutrinos, as measured by SNO and Super-Kamiokande (solid line) and as predicted by the SSM 


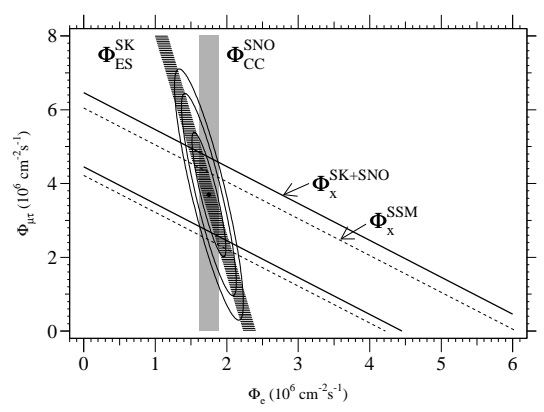

Fig. 10. Correlation between the non-electron active component of the solar ${ }^{8} \mathrm{~B}$ flux, $\Phi_{\mu \tau}$, and the electron component $\Phi_{e}$. The two shaded bands represent the $1 \sigma$ bars of the neutrino fluxes as obtained from SNO CCB and Super-Kamiokande ES measurements. 32 The ellipses are the 68\%, $95 \%$ and $99 \%$ joint probability for the two fluxes. The two diagonal bands are the measured $(\mathrm{SK}+\mathrm{SNO})$ and predicted (SSM) 23 bands of constant total flux of active ${ }^{8} \mathrm{~B}$ neutrinos.

model (dashed line). The measured value, $\Phi\left(\nu_{x}\right)=(5.44 \pm 0.99) \times 10^{6} \mathrm{~cm}^{-2} \mathrm{~s}^{-1}$, is in very good agreement with predictions.

In conclusion, SNO has presented a first direct indication for a non-electron active component in the solar neutrino flux. Moreover the experiment measures a ${ }^{8} \mathrm{~B}$ neutrino flux consistent with the SSM expectations. These are only the first of several very interesting measurements that SNO should be able to perform over the next few years: we now look forward to the results from the experiment's second phase.

Borexino, 39 a 300 ton liquid scintillator detector with PMT readout, is being installed at the Gran Sasso Underground Laboratory. Thanks to the high radiopurity and to the high light yield of the scintillator, it will be able to detect neutrinoelectron scattering with an energy threshold as low as $250 \mathrm{keV}$. This means that the ${ }^{7} \mathrm{Be}$ lines, especially that at $0.863 \mathrm{MeV}$, will be within the reach of the experiment. The monochromaticity of the beryllium line should facilitate the observation of VO-induced variation of the solar neutrino flux, which for Borexino would be a spectacular effect. Moreover Borexino will also be able to check the LOW solution, by studying the day/night effect for beryllium neutrinos, which is expected to be strong if indeed oscillations occur with the LOW parameters 42 Borexino is expected to start data-taking in 2002 .

A test of the LMA solution will be made by the KamLAND 40 experiment, a 1 kton liquid scintillator detector, located in the Kamioka mine in Japan. The experiment will detect the low-energy $(1-8 \mathrm{MeV}) \bar{\nu}_{e}$ flux emitted by the nuclear reactors at several Japanese power stations, at distances between 150 and $210 \mathrm{~km}$ from the detector. The estimated sensitivity of KamLAND is such that, at $90 \%$ C.L. and after three years of data taking, the experiment should be able to cover the entire domain defined by the LMA solution (down to $\Delta m^{2} \sim 4 \times 10^{-6} \mathrm{eV}^{2}$ ). If oscillations are observed, the oscillation parameters should be determined to a precision of $20 \%$, 
at $99 \%$ C.L.. KamLAND is expected to start data-taking at the end of this year.

More experiments are planned to study solar neutrinos in the future. Among them one should certainly mention ICARUS 43 a liquid-argon TPC detector, which will be mainly sensitive to ${ }^{8} \mathrm{~B}$ and of which the first 600 tons half-module is currently under test, HERON,44 which plans to study $p p$ and beryllium neutrinos using superfluid helium as a target, and HELLAZ, 15 which intends to study low-energy neutrinos, down to the $p p$ neutrino energies, using a gaseous helium TPC.

In the previous sections we have outlined the solar neutrino problem, which historically represents the first experimental hint for neutrino oscillations. We have described the standard solar model results and the arguments which make the model convincing and robust. We have discussed how experimental data are in striking contradiction with expectations, if no new physics is taken into account, and we have summarize the results obtained when the solar neutrino deficit is interpreted as being due to neutrino oscillations. We now want to plunge into another exiting domain, that of atmospheric neutrinos, which undoubtedly provide the most convincing experimental evidence for neutrino oscillations.

\section{The Atmospheric Neutrino Anomaly}

\subsection{Atmospheric Neutrino Flux and Neutrino Oscillations}

Atmospheric neutrinos are produced in the decay of secondary particles created in the interaction of primary cosmic rays ${ }^{j}$ with the Earth's atmosphere. If the energy of the secondary particles is sufficiently low $(\lesssim 2 \mathrm{GeV})$ that all of them decay, we have:

$$
\begin{aligned}
p+\mathcal{N} & \longrightarrow \pi^{ \pm}+X \\
\pi^{ \pm} & \longrightarrow \mu^{ \pm}+\nu_{\mu}\left(\bar{\nu}_{\mu}\right) \\
\mu^{ \pm} & \longrightarrow e^{ \pm}+\nu_{e}\left(\bar{\nu}_{e}\right)+\bar{\nu}_{\mu}\left(\nu_{\mu}\right)
\end{aligned}
$$

Assuming that no effect can change the flavour composition of the shower before it is measured on Earth, Eq.(45) implies that:

$$
\mathcal{R}=\frac{\mathcal{N}_{\nu_{\mu}}+\mathcal{N}_{\bar{\nu}_{\mu}}}{\mathcal{N}_{\nu_{e}}+\mathcal{N}_{\bar{\nu}_{e}}} \sim 2
$$

where $\mathcal{N}_{\nu_{\mu}}\left(\mathcal{N}_{\nu_{e}}\right)$ and $\mathcal{N}_{\bar{\nu}_{\mu}}\left(\mathcal{N}_{\bar{\nu}_{e}}\right)$ are the number of muon (electron) neutrinos and anti-neutrinos respectively. The exact value of $\mathcal{R}$ can in principle be affected by several effects, such as the primary spectrum composition, the geomagnetic cut-off, solar activity and, of course, the details of the model for the development of the hadronic shower. However, it has to be said that, although the absolute neutrino fluxes are rather badly known (predictions from different calculations disagree by $\sim 20-30 \%$ ), the ratio (46) is to first order insensitive to such uncertainties and is

\footnotetext{
$\overline{j \text { mainly protons }(\sim 80 \%) \text { and } \alpha \text {-particles }}(\sim 15 \%)$, plus a small contribution from heavier nuclei. 16
} 
known to $\sim 5 \%$. Neutrino oscillations would manifest themselves as a discrepancy between the measured and the expected value of the ratio $\mathcal{R}$.

In order to quote a number which is independent of experimental parameters, such as energy thresholds and detector acceptances for signal and background, the result is generally presented in terms of the double ratio:

$$
\mathcal{R}^{\prime} \equiv \frac{\mathcal{R}_{D A T A}}{\mathcal{R}_{M C}}=\frac{\left[\frac{\mathcal{N}_{\nu_{\mu}}+\mathcal{N}_{\bar{\nu}_{\mu}}}{\mathcal{N}_{\nu_{e}}+\mathcal{N}_{\bar{\nu}_{e}}}\right]_{D A T A}}{\left[\frac{\mathcal{N}_{\nu_{\mu}}+\mathcal{N}_{\bar{\nu}_{\mu}}}{\mathcal{N}_{\nu_{e}}+\mathcal{N}_{\bar{\nu}_{e}}}\right]_{M C}}
$$

where $\mathcal{R}_{D A T A}$ is the measured ratio of muon-like (i.e. $\nu_{\mu}, \bar{\nu}_{\mu}$ ) over electron-like (i.e. $\nu_{e}, \bar{\nu}_{e}$ ) events and $\mathcal{R}_{M C}$ is the same ratio as obtained from a Monte Carlo simulation. Assuming a correct modelling for $\mathcal{R}_{M C}, \mathcal{R}^{\prime}<1$ could either mean that there is a deficit of measured muon-like events, or that an excess of electron-like events has been observed. The quantity (47) alone cannot discriminate between these two possibilities. Moreover, a low value of $\mathcal{R}^{\prime}$ is not by itself a proof for neutrino oscillations, since one could imagine other mechanisms (i.e. neutrino decay) inducing a similar effect.

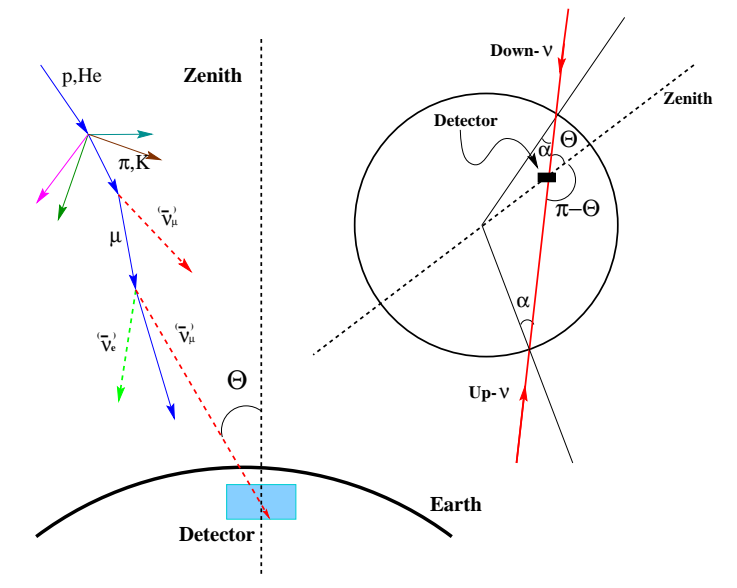

Fig. 11. Sketch of the atmospheric neutrinos detection principles.

Another, more sensitive way to detect neutrino oscillations is the study of the zenith angle distribution of the incoming atmospheric neutrino. Downward-going neutrinos, produced in the atmosphere above the detector, will travel a path of the order of $10-20 \mathrm{~km}$, while upward-going neutrinos, produced at the opposite side of the globe, will have travelled up to $12,000 \mathrm{~km}$ before detection. This means that, by studying the neutrino flux as a function of the zenith angle, one has access to 
baselines spanning three orders of magnitude and can therefore hope to observe the modulation induced on the flux by neutrino oscillations.

If no oscillation occurs between the production point and the detector, assuming for simplicity that no other effect alters the cosmic ray angular distribution, the neutrino flux per unit area is the same at any location on the Earth. If we consider the contribution to the flux from a point at zenith angle $\Theta$ (downward-going neutrinos) and we compare it to the flux at zenith angle $\pi-\Theta$ (upward-going neutrinos), based on simple symmetry considerations (see Fig.11), we can demonstrate that the two quantities must have the same value in the absence of new physics. In summary, in the no-oscillation hypothesis, the zenith angle distribution must be up-down symmetric, assuming no other phenomena affecting the neutrino angular distribution relative to the local vertical direction. Conversely, any deviation from up-down symmetry could be interpreted as an indication for neutrino oscillations.

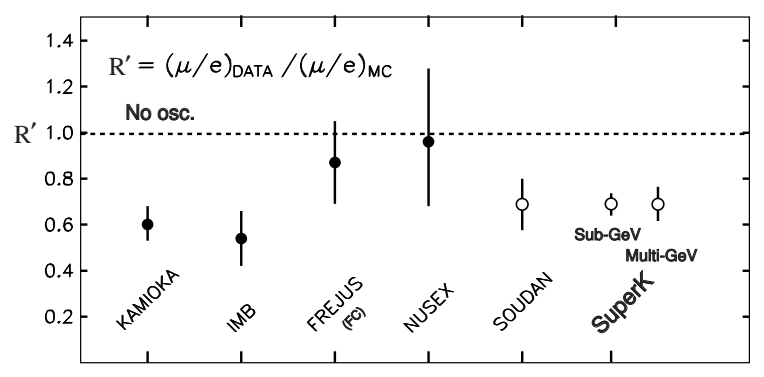

Fig. 12. Compilation of the $\mathcal{R}^{\prime}$ ratio (see text) measured by several atmospheric neutrino experiments (from ref.4t).

Historically, the first results on what is nowadays known as the atmospheric neutrino anomaly came from experiments originally designed to search for proton decay, for which the atmospheric neutrino flux constituted a background source. A compilation of results on $\mathcal{R}^{\prime}$ from different experiments is shown in Fig.12 (from ref.47): all measurements are systematically below one and all but two give a value for $\mathcal{R}^{\prime}$ close to 0.7 .

The first experiment to report, in 1986, a discrepancy between the observed and the predicted number of atmospheric neutrinos, although that was not_immediately recognised as a possible effect of neutrino oscillations, was IMB 48 a water-Cherenkov detector located in the Morton mine, Cleveland, Ohio, USA. Two years later Kamiokande confirmed that the measured deficit of muon-like events was the order of $30 \% .49$ However, the same effect was not observed by two other proton decay experiments, NUSEX 50 and Fréjus, 1 , both using fine-grained iron calorimeters. This discrepancy led for some time to the belief that poorly understood systematic effects, mainly related to an incomplete description of neutrino interactions in iron and water, were inducing an intrinsic difference between the two experimental techniques. Although later calculations had already shown that 
the neglected physical processes could account for at most a $10-15 \%$ change in the expected event rates 52 the issue was only definitively resolved when the Soudan2 experiment, 53 another fine-grained iron calorimeter, located in the Soudan mine (2100 m.w.e.), Ely, Minnesota, USA, confirmed Kamiokande and IMB results. It is now common belief that the original discrepancy was due to fluctuations in NUSEX and Fréjus data. In 1994 Kamiokande showed a distortion of the zenjth angle distribution in the multi-GeV contained events (see below for a definition), 54 which seemed to confirm the neutrino oscillation hypothesis. Kamiokande and IMB as well as Baksan,55 an experiment in the former Soviet Union, and MACRO 56 a proton decay experiment at the Gran Sasso Laboratory, also tried to extract information from the sample of particles travelling in the vertical direction, but the results were not consistent with each other and none of them seemed conclusive.

The big change in the scientific community's perception of the atmospheric neutrino anomaly certainly came from the results obtained by the Super-Kamiokande experiment, 57,58,59 which in summer 1998 announced evidence for neutrino oscillations in the atmospheric neutrino sample. However, before we discuss atmospheric neutrinos in Super-Kamiokande, we shall first present some of the ideas and the status of the atmospheric neutrino flux calculations.

\subsection{Atmospheric Neutrino Flux Calculations}

The neutrino flux on Earth (approximately 1 event $/ \mathrm{cm}^{2} / \mathrm{sr} / \mathrm{sec}$ ) can in principle be calculated either starting from the spectrum of the primary protons and alpha particles which initiate the hadronic cascade in the atmosphere, or from the measured muon flux at high altitudes. In the first case it is necessary to parametrize the hadronic shower development, thus requiring a relatively good understanding of the pion production process at the relevant energies. On the other hand, the muon acceptance has the disadvantage of being quite sensitive to variations of the geomagnetic field. Moreover the exposure time for muon flux measurements is quite short, the relevant experiments being generally performed on balloons, 6061.62 while primary fluxes measurements, made at high altitudes or above the atmosphere, are usually made over relatively longer intervals.60.63.64.65.66 In any case a measurement of the muon flux gives a useful constraint on the the atmospheric neutrino flux calculations.

At low geomagnetic latitudes the trajectories of low energy primary cosmic rays can be significantly deviated under the action of the geomagnetic field and, in extreme cases (energies of the order of few $\mathrm{GeV}$ ), they would not reach the atmosphere at all. At intermediate energies $(10-20 \mathrm{GeV})$, the flux of primary cosmic particles exhibits a strong east-west asymmetry, while at high energies the flux is essentially unaffected by the terrestrial magnetic field. In summary, the net effect of the geomagnetic field is a non-isotropy of the neutrino flux even in the absence of oscillations. This has been observed by Super-Kamiokande 58 which has measured an east-west asymmetry (see Fig.13, from ref.58) consistent with expectations, thus confirming that the details of the geomagnetic effects on the cosmic radiation are 


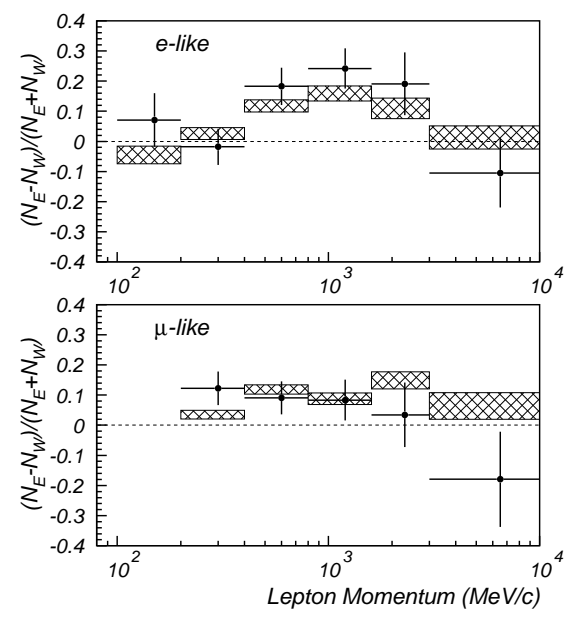

Fig. 13. East-west asymetry measured by Supqramiokande 88 as a function of the lepton momentum (from ref. 8 ), compared to expectations. $0 N_{E}$ and $N_{W}$ are the number of events from the east and the west respectively.

well understood. In Super-Kamiokande (geomagnetical latitude $25.8^{0} \mathrm{~N}$, close to the geomagnetic equator) the cutoff momentum for protons arriving horizontally from the east is $\sim 50 \mathrm{GeV}$.

A modulation of the low energy component of the primary cosmic ray flux is expected as a function of the solar activity (cycle of $\sim 11$ years), which induces fluctuations in the geomagnetic field (see for example ref.67): the greater the solar activity, the lower the primary cosmic ray flux on the Earth. The correlation between the relative sun-spot number and the cosmic ray flux can be studied using the data from the Mount Washington neutron monitor 68 The influence of the solar wind is only sizeable $(\sim 15-20 \%)$ for neutrino energies of $1 \mathrm{GeV}$ or below and only at high geomagnetic latitudes, where the geomagnetic effect is minimal, while it becomes negligible at $\sim 2 \mathrm{GeV} 69$

The experimental results for atmospheric neutrinos are usually presented in terms of the neutrino flux calculations performed by Honda and collaborators (HKKM) 80 and by the Bartol group 71 A comparison between the two models and a compilation of some of the most recent data on primary protons is shown in Fig.14 (from ref 72 ).

Both HKKM and the Bartol models are based on one-dimensional computations, in the sense that the momenta of all secondary particles, hadrons, charged leptons as well as neutrinos, are assumed to be collinear with that of primary cosmic ray (1-D calculation). It has only very recently been realized that three-dimensional (3-D) calculations can be important for a correct description of the neutrino spatial 


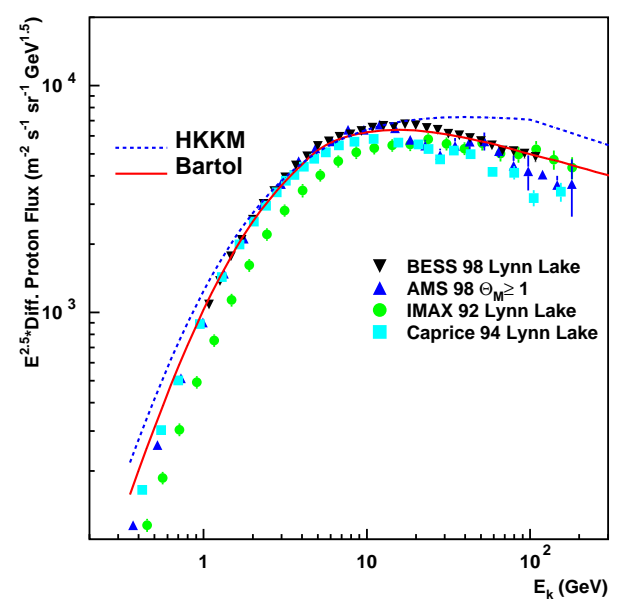

Fig. 14. Comparison between the HKKM 70 and the Bartol71 flux calculations with some of the most recent data on primary protons (from ref. 22 ).

distribution, especially in the sub-GeV energy range.737.75 The new calculations, originally motivated by the need of having a better description of the hadronic processes inglved in the shower development, are based on the the FLUKA simulation package. 6 The effect of taking the three-dimensional development of the shower at low energies $(\lesssim 1 \mathrm{GeV})$ can be clearly seen in Fig.15, where the results from FLUKA-based 1-D and 3-D simulations are shown for the zenith angle distribution at the Kamioka site (figure from ref 73 ).

The Bartol and FLUKA groups have started a comparison of the two models, using the same input primary spectrum 72 The results of this study show that the two normalisations disagree by approximately $20 \%$ (Bartol is lower) and the difference between the two models appears to be energy-dependent. According to the author of ref.22, a preliminary comparison with HKKM shows that, if they had used the same primary spectrum as an input, also their predictions on the normalisation would have been lower than FLUKA's.

In summary, even if the $e / \mu$ ratio is rather well known, the uncertainties on the absolute neutrino fluxes are still quite large. Among the dominant sources of systematic errors are the knowledge of the primary fluxes, still limited despite new data has been recently made available, and the uncertainties on the secondary particle production model. Other effects, such as the atmosphere modelling and the detector altitude, play only a marginal role. In order to improve the theoretical calculations it is very important that further constraints on the models are obtained from new experimental data.

Several experiments relevant for the atmospheric neutrino flux calculations should be taking data over the next few years. HARP, 7 an experiment running at the CERN PS, aims to study hadroproduction in a range of energy relevant for future 


\section{Sub-GeV flux at Kamioka}
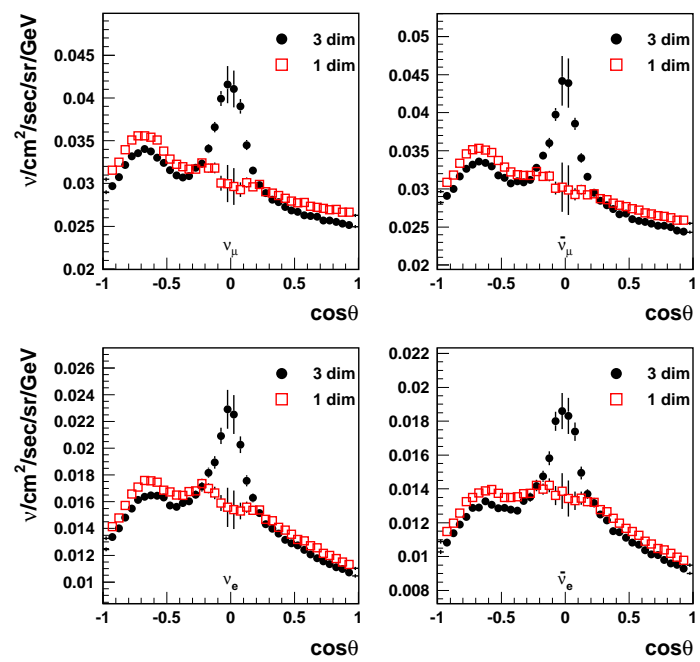

Fig. 15. Comparison between 1-D and 3-D pedictions for the neutrino fluxes at the Kamioka site, as a function of the zenith angle (from ref. 3 ).

projects such as neutrino factories and for the determination of the atmospheric neutrino flux. More recently a proposal for another experiment has been submitted to CERN: 8 if approved, that experiment would make use of the NA4979 apparatus to study hadroproduction in a range of energies $(120 \mathrm{GeV})$ relevant for both atmospheric neutrinos and long-baseline neutrino oscillation projects.

Among the new proposals to study cosmic ray muons for the determination of the neutrino fluxes, an interesting project, known as ADLER 80 is now in its design stage. The main idea is to fly a small detector on board an aircraft to measure the atmospheric muon flux at different geomagnetic latitudes. This possibility, together with the relatively longer exposure times, would be an obvious advantage with respect to balloon-borne experiments.

\subsection{Atmospheric Neutrinos in Super-Kamiokande}

We have already given a brief description of the Super-Kamiokande experiment and will not repeat it here, however it is worth summarising some information relevant for the following discussion. Atmospheric neutrinos are detected in SuperKamiokande by measuring the Cherenkov rings generated by the primary particles produced in the neutrino CC interactions with the water nuclei. Thanks to the high PMT coverage, the experiment is characterised by an extremely good light yield $(\sim 8$ photo-electron per $\mathrm{MeV}$ ) and can detect events of energies as low as $\sim 5 \mathrm{MeV}$. The 
large detector mass and the possibility of clearly defining a large inner volume allow to collect a high statistics sample of fully contained events (FC) of relatively high energies (up to $\sim 5 \mathrm{GeV}$ ), the FC events being defined as those having both the neutrino vertex and the resulting particle tracks entirely within the fiducial volume. The contamination from downward-going cosmic muons is drastically reduced by the containment requirement on the primary vertex coordinates. Fully contained events can be further subdivided into two subsets, the so-called sub-GeV and multi-GeV events, with energies below and above $1.33 \mathrm{GeV}$ respectively. In Super-Kamiokande jargon FC events include only single-ring events, while multi-ring ones (MRING) are treated as a separate category. Another sub-sample, defined as the partially contained events (PC), is represented by those charged-current interactions where the vertex is still within the fiducial volume, but at least a primary particle, typically the muon, exits the detector without releasing all of its energy: for those events the energy resolution is therefore worse than for FC interactions. Finally, upwardgoing muons (UPMU), produced by neutrinos coming from below and interacting in the rock, can also be used to independently check the neutrino oscillation result. In the literature they are sometimes further subdivided into stopping muons and through-going muons, according to whether or not they stop in the detector. The different samples defined above explore different ranges of the neutrino energy: this is shown in Fig. 16 (from ref.82), where the event rates for sub-GeV and multi-GeV FC events, as well as for through-going and stopping muons is shown. For PC events the neutrino energy is in the multi-GeV range.

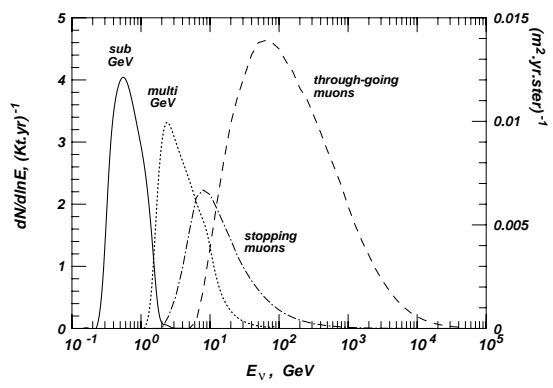

Fig. 16. Event rates as a function of the neutrino energy for some of the different categories of neutrino interactions defined in the text (fig. from ref.82).

Particle identification in Super-Kamiokande is performed using likelihood functions to parametrize the sharpness of the Cherenkov rings, which are more diffused for electrons than for muons. The algorithms, which have been tested on cosmic muons, decay electron samples and using test-beam data, 81 are able to discriminate the two flavours with very high purity (of the order of $98 \%$ for single track events).

The most recent value for $\mathcal{R}^{\prime}$ reported by the Super-Kamiokande collaboration, based on 1289 days of data, is $0.638_{-0.017}^{+0.017} \pm 0.050$ for the sub-GeV sample and 
$0.675_{-0.032}^{+0.034} \pm 0.080$ for the multi-GeV sample (both FC and PC). 33 Thus the measured value of $\mathcal{R}^{\prime}$ is different from unity by about $7 \sigma$.
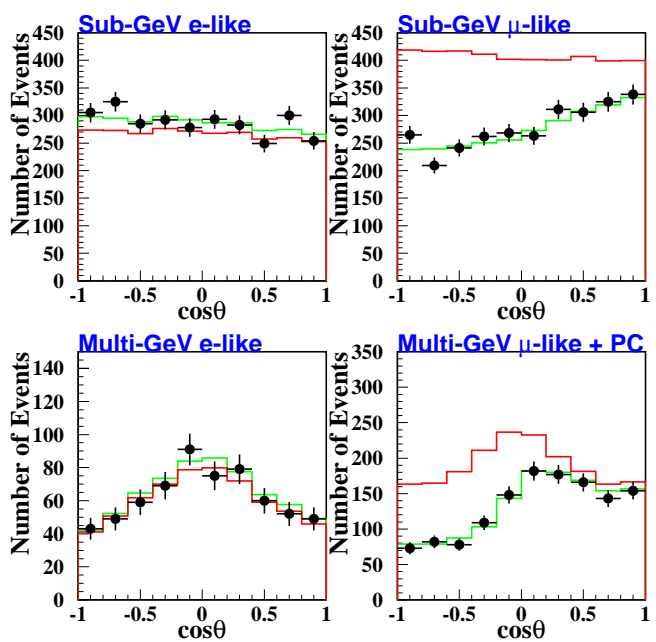

Fig. 17. Distribution of the cosine of the zenith angle obtained by Super Kamiokade from 1289 live days data, for electron-like and muon-like contained events (from ref 84 ). The solid line is the distribution expected in absence of oscillations, while the hatched histogram is for $\nu_{\mu} \rightarrow \nu_{\tau}$ oscillations in the two-flavour mixing scheme, with maximal mixing and $\sin ^{2} 2 \theta=2.5 \times 10^{-3} \mathrm{eV}^{2}$.

The distributions of the cosine of the zenith angle for the sub-GeV and the multi-GeV samples are shown in Fig.17 (1289 live days, from ref 84 ). In both subsets the electron-like events are in good agreement with predictions in absence of oscillations, while the muon spectrum, which strikingly disagrees with no-oscillation expectations, is well described by an oscillation induced modulation. It has to be noticed that, while the sub-GeV muon sample is depleted over the entire spectrum, the deficit being larger for smaller values of $\cos \Theta$, the spectrum of neutrinos coming from above $(\cos \Theta>0)$ is very little changed in the case of multi-GeV muons. This is consistent with $\nu_{\mu}$ oscillations occurring with a value of $\Delta m^{2}$ which is better matched by low-energy neutrinos (sub-GeV), while for high energy neutrinos the effect of oscillations becomes sizeable only for larger baselines (neutrinos coming from below). However it has to be stressed that the angular resolution degrades at lower energies, where the lepton momentum direction becomes a worse approximation for the incoming neutrino direction.

A useful dimensionless quantity which can be derived from the zenith angle distribution is the so-called up-down asymmetry $A$, defined as the difference between the number of upward-going $(\mathrm{U}, \cos \Theta<-0.2)$ and the number of downward-going 
$(\mathrm{D}, \cos \Theta>0.2)$ neutrinos, divided by the sum of the two numbers:

$$
A=\frac{U-D}{U+D} .
$$

We have seen that, neglecting the geomagnetic effect corrections, which can be taken into account in the simulation, there is no reason to have any asymmetry in the neutrino flux. Any deviation of $A$ from zero is a signature for new physics and can be interpreted in terms of neutrino oscillations. The dependence of this quantity on the measured particle momentum is shown in Fig.18 (left) for electron-like and muon-like events (both FC and PC). The uncertainty assumed on the absolute normalisation is $25 \%$, with $20 \%$ contribution from the neutrino flux calculation and $15 \%$ from the neutrino interaction cross section. Also here the experimental data are consistent with neutrino oscillations in the muon sample, while electron neutrinos appear to be unaffected. In agreement with the oscillation hypothesis, we see that for muon-like events the asymmetry is essentially zero at low energies, where the oscillation length is small and therefore both upward-going and downward-going muons are equally depleted, while for increasing values of the muon momentum, when mostly neutrinos from below are affected by the oscillation, the asymmetry becomes more and more negative.
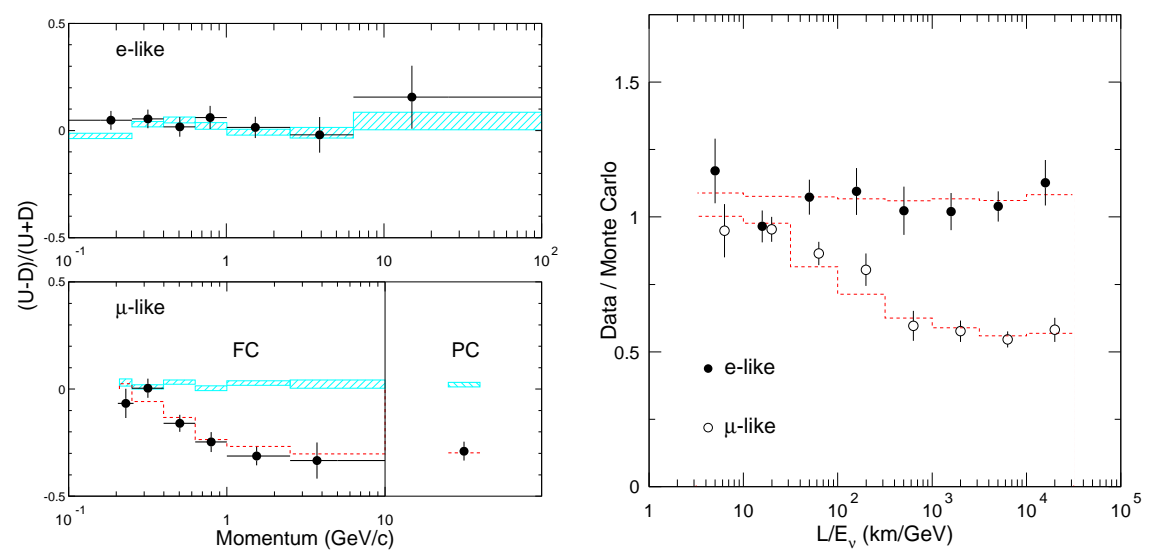

Fig. 18. Super-Kamiokande (1144 days) up-down asymmetry for electron-like and muon-like events, as a function of the particle momentum(left) and ratio between observed and expected number of electron-like and muon-like events as a function of $L / E$ (right). The dashed lines correspond to $\nu_{\mu} \rightarrow \nu_{\tau}$ oscillations with maximal mixing and $\Delta m^{2}=3.2 \times 10^{-3} \mathrm{eV}^{2}$, while the hatched boxes indicate no-oscillation expectations (figures from ref.59).

Another way of looking at the same effect is to consider the ratio between observed and expected number of electron-like and muon-like events as a function of the ratio $L / E$ : this is shown in Fig.18 (right) for 1144 live day of Super-Kamiokande data. Also here electron-like events show no evidence for oscillations, while muonlike events show a dependence on $L / E$ which, although the oscillatory pattern is 
washed out by angular and energy resolution, is consistent with the oscillations hypothesis.

Interpreting the muon-like event deficit as the result of $\nu_{\mu} \rightarrow \nu_{\tau}$ oscillations in the two-flavour mixing scheme, Super-Kamiokande extract an allowed domain for the oscillation parameters. Events are binned in a multi-dimensional space defined by particle type, energy and zenith angle, plus a set of parameters to account for systematic uncertainties. The result of the fit based on 1289 days of data, using FC, PC, UPMU and MRING events, is given in Fig.19 _eft. The best fit corresponds to maximal mixing and $\Delta m^{2}=2.5 \times 10^{-3} \mathrm{eV}^{2} .4$ For comparison, an earlier plot $^{k}$ of Super-Kamiokande allowed region (90\% C.L.) is shown together with those obtained by Kamiokande, 19 , Soudan2, 55 and MACRO 86 In particular it is interesting to consider how the preferred solution for $\Delta m^{2}$ indicated by SuperKamiokande is lower than that previously presented by Kamiokande, with only a very small overlap between the $90 \%$ C.L. contours obtained by the two experiments. The reason for this effect is not fully understood, although one possible explanation could be the different fitting procedure, which for Kamiokande is based on the flavour ratio $\mathcal{R}^{\prime}$ only, while in Super-Kamiokade a shape analysis of the energy and the zenith angle spectra is performed.

The Soudan 25 and the MACRO 86 experiments, although with less sensitivity, have confirmed an atmospheric neutrino anomaly consistent with that observed by Super-Kamiokande.
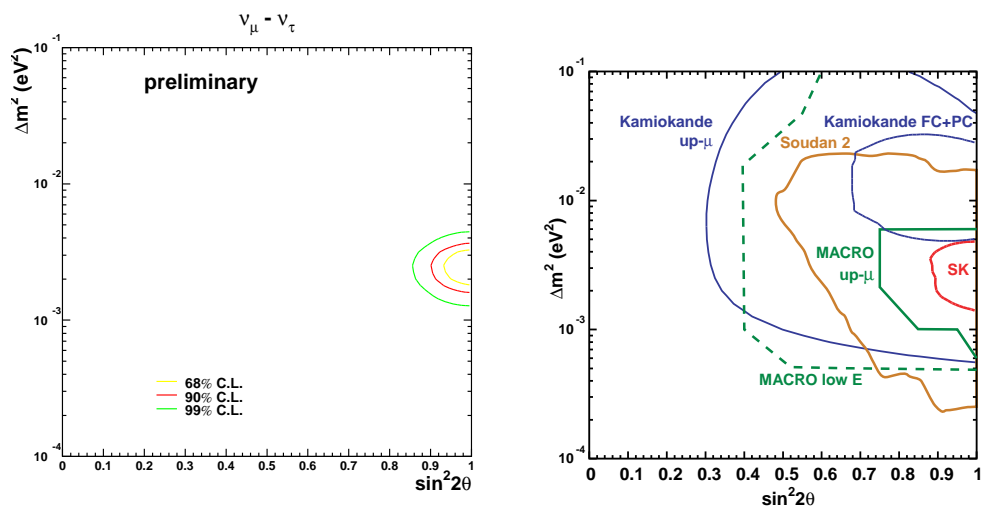

Fig. 19. (Left) Allowed region at different C.L. aptained by Super-Kamiokande (1289 days) in the two-f your $\nu_{\mu} \rightarrow \nu_{\tau}$ hypothesis (f ref 84 ). (Right) An exilier contour from SuprKamiokande 87 is compared to Kamiokande, 199 , Soudan2 85 and MACRO 86 results (fig. from ref. 67 ).

The Super-Kamiokande interpretation of the atmospheric neutrino anomaly in terms of muon disappearance rather than electron appearance is corroborated by

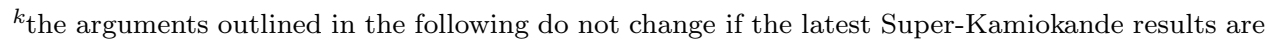
considered.
} 
the results obtained by CHOOZ 88 and Palo Verde 89 two neutrino oscillation experiments at nuclear reactors, which have studied $\bar{\nu}_{e}$ disappearance in the parameter region relevant for atmospheric neutrinos. In particular CHOOZ limits (at 90\% C.L., $\Delta m^{2}<7 \times 10^{-4}$ for maximum mixing and $\sin ^{2} 2 \theta<0.10$ for large $\Delta m^{2}$ ) seem to exclude $\nu_{\mu} \leftrightarrow \nu_{e}$ mixing in the region of interest, at least as a dominant mode. 90 However the ambiguity between oscillations to tau or sterile neutrinos is not resolved by the analysis of Super-Kamiokande data described previously. In order to discriminate between the $\nu_{\mu} \rightarrow \nu_{\tau}$ and the $\nu_{\mu} \rightarrow \nu_{s}$ hypotheses, it is necessary to consider other physics effects able to distinguish one mode from the other. First of all, while the $\nu_{\tau}$ has standard neutral current interactions with matter, $\nu_{s}$ does not couple to the $Z^{0}$ boson, with a consequent depletion of $\mathrm{NC}$ events and an up-down asymmetry in the NC sample. Moreover, in case of mixing with a sterile neutrino, matter effects - see Eqs.(26), (27) and (28) - would induce a sizeable change in the oscillation probability for neutrino energies such that $E /\left|\Delta m^{2}\right| \gtrsim 10^{3} \mathrm{GeV} / e \mathrm{~V}^{2} .11$ Based on those considerations, Super-Kamiokande has published an analysis of 1100 live days data, showing that the $\nu_{\mu} \rightarrow \nu_{\tau}$ hypothesis fits lowenergy CC interactions, but does not fit the NC sample or high energy CC data.92 Although they cannot exclude more complicated schemes in which the mixing is between $\nu_{\mu}$ and admixture of $\nu_{\tau}$ with a small component of $\nu_{s}$, pure $\nu_{\mu} \rightarrow \nu_{s}$ is disfavoured by their data at $99 \%$ C.L.. Another way of testing the $\nu_{\mu} \rightarrow \nu_{s}$ hypothesis, which is again motivated by the $\nu_{s}$ not having standard electro-weak interactions, is to look for possible suppressions of $\pi^{0}$ production. This technique is currently limited by the systematic error on the $\pi^{0}$ production cross-section, but the situation should improve in the near future, since the K2K experiment 93 which we shall discuss shortly, is expected to reduce the uncertainty from the present value of $\sim 20 \%$ down to $\sim 5 \%$.

Super-Kamiokande has recently presented preliminary results on $\nu_{\tau}$ appearance. 84 The experiment does not have the resolution to unambiguously identify an event as being a $\nu_{\tau} \mathrm{CC}$ interaction, however they have developed a likelihood-based analysis to extract a sample of tau-like events. The observed number of selected events is 419 , for an expected number of $31 \nu_{\tau}$ 's plus 387 background events; moreover the measured zenith angle distribution is better fitted by $\nu_{\tau}$ 's plus background than by background only.

In summary, we have seen that Super-Kamiokande results, which have been confirmed with less sensitivity by other atmospheric neutrino experiments, represent a strong hint for neutrino oscillations with near-to-maximal mixing $\left(\sin ^{2} 2 \theta>0.88\right)$ and $\Delta m^{2}$ in the range $2-6 \times 10^{-3} \mathrm{eV}^{2}$. New experiments, like ICARUS43 and MONOLITH, 94 will study or plan to study atmospheric neutrinos in the future. However the uncertainties on the atmospheric neutrino flux predictions are such that a firmer experimental proof of neutrino oscillations is required, together with a better determination of the oscillation parameters if the effect is confirmed. For this reason it is very important to check the atmospheric neutrino anomaly in controlled experimental conditions, by using artificially produced neutrinos (i.e. accelerator beams). 
Typical neutrino energies at accelerators are in the range $\sim 1-30 \mathrm{GeV}$, therefore, in order to match the $\Delta m^{2}$ domain suggested by atmospheric neutrino results, it is necessary to increase the detector-to-source separation to distances of the order of hundreds of kilometres. Experiments of this sort are commonly referred to as long-baseline experiments and we shall discuss them in the next section.

\subsection{Long-baseline Accelerator Experiments}

The first accelerator-based long-baseline neutrino oscillation experiment ever built is K2K (KEK-to-Kamioka) 93 which makes use of an almost pure $\nu_{\mu}$ beam (98.2\%) directed from KEK, in Japan, to the Super-Kamiokande detector, $250 \mathrm{~km}$ away. K2K, which has been taking data since June 1999, is a disappearance experiment, aiming to measure a deficit of muon neutrinos in the far detector at Kamioka compared to the initial beam intensity measured in the near detector at KEK. The neutrino beam, produced in the interactions of $12 \mathrm{GeV}$ primary protons from the KEK-PS on an aluminium target, has an average energy of $\sim 1.3 \mathrm{GeV}$ (hence the maximum sensitivity to oscillations is for $\Delta m^{2} \sim 5 \times 10^{-3} \mathrm{eV}^{2}$ ). Pion and muon detectors, placed along the beamline, are used to measure the secondary particles after the horn focusing system and to monitor the beam centering and its intensity. The design proton intensity is $6 \times 10^{12}$ protons per pulse $(1.1 \mu \mathrm{s}$ spill/2.2 s period). The results recently presented by the $\mathrm{K} 2 \mathrm{~K}$ collaboration are based on $2.29 \times 10^{19}$ pot (protons-on-target), collected between June 1999 and June 2000. The alignment between the neutrino target and the far detector is performed using a GPS survey, giving a precision better than $0.01 \mathrm{mrad}$, while the precision of the civil construction is better than $0.1 \mathrm{mrad}$. GPS timing information is also used to correlate events in Super-Kamiokande with the KEK-PS spill.

In order to have a reliable measurement of the beam intensity at production and to reduce the systematic uncertainties related to the experimental technique adopted, the near detector has been built using the same principles and technology as for Super-Kamiokande. Located $300 \mathrm{~m}$ from the production point, it is a 1 kton water-Cherenkov detector with photo-multiplier readout, the PMTs being arranged so to provide the same coverage as for Super-Kamiokande (40\%). The water-Cherenkov detector is complemented by a scintillating fibre tracker (with a 6 ton water target), a lead-glass electromagnetic calorimeter and a muon range detector. The combined information from the different sub-detectors allows measurement of the $\nu_{\mu}$ beam profile, its energy distribution and intensity, as well as the determination the $\nu_{e}$ contamination $(\sim 1.3 \%)$ and the study of neutrino interactions in the $1 \mathrm{GeV}$ region.

The beam has been measured to be centred within systematic uncertainties $(0.7 \mathrm{mrad})$ and the centre has been shown to be stable within $\pm 1 \mathrm{mrad}$. The muon energy and angular distributions are also monitored on a daily basis and so is the beam intensity: they all appear to be stable over the period of data taking considered. Given this stability, it is possible to predict the number of expected events at the far site in absence of oscillations by extrapolating the normalisation 
measured at the near site to the far detector location. The beam simulation used for the extrapolation is validated by a comparison between the predicted secondary pion momentum and angular distributions and those measured at the pion monitor placed along the beamline. The selection criteria used to identify muon-like and electron-like interactions at the near detector are essentially the same as those used in Super-Kamiokande for the FC atmospheric neutrino analysis, with the addition of a cut on the total number of photoelectrons to discard spills with multiple events.

Table 5. Number of expected and observed events in the far detector of the K2K experiment.

\begin{tabular}{ccc}
\hline Sample & Expected Events (No osc.) & Observed Events \\
\hline 1-ring $\mu$-like & 20.9 & 14 \\
1-ring $e$-like & 2.0 & 1 \\
MRING & 14.9 & 13 \\
\hline Total & $37.8 \pm 0.2(\text { stat. })_{-3.8}^{+3.5}($ sys. $)$ & 28 \\
\hline
\end{tabular}

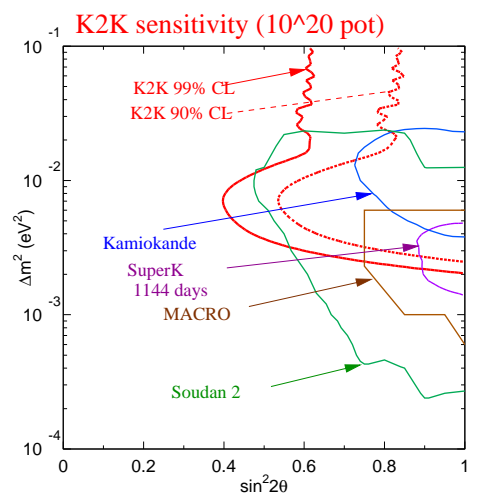

Fig. 20. K2K expected sensitivity for $10^{20}$ pot, compared with the allowed regions in the oscillation parameter space obtained by Super-Kamiokande, Soudan2 and MACRO (from ref 95 ).

The systematic uncertainty on the near detector flux measurement is $5 \%$, with a statistical error of $<1 \%$. For the far detector the systematic error is $3 \%$, while the uncertainty related to the near-to-far extrapolation is estimated to be ${ }_{-7}^{+6} \%$. Other uncertainties, such as those on the beam spectrum and the neutrino interaction cross sections, are negligible to first order, due to cancellations in the near-to-far comparison. The expected number of events in absence of oscillations is $37.8 \pm 0.2(\text { stat. })_{-3.8}^{+3.5}$, while only 28 have been observed in real data. In Tab. f the predicted and observed number of events are compared for the different categories (one-ring muon-like, one-ring electron like and multi-ring): while essentially no conclusion can be drawn about electron-like events, due to the small statistics, the muon neutrino sample appears to be significantly depleted, a fact which might be interpreted as evidence for neutrino oscillations. However, the K2K collaboration has not yet given any in- 
terpretation of their result and is waiting to have more statistics $\left(10^{20}\right.$ pot by year 2005) and a better understanding of systematic uncertainties before drawing any strong conclusion from the observed anomaly. The expected final sensitivity for K2K is shown in Fig.20, together with the allowed solutions obtained by Kamiokande, Super-Kamiokande, MACRO and Soudan2. From that plot it becomes clear that, unless the actual $\Delta m^{2}$ is at the high end of Super-Kamiokande allowed region, K2K will not provide a definitive proof that the atmospheric neutrino anomaly is due to neutrino oscillations.

Two other long-baseline projects are expected to start operation over the next few years: one in the USA, the MINOS 96 experiment at Soudan, using the NuMI 97 beam from Fermilab, and the other in Europe, using the CERN-to-Gran Sasso neutrino beam 98 (NCGS) in conjunction with the two proposed experiments OPERA99 and ICARUS/ICANOE. 13.100

The MINOS experiment aims to investigate the atmospheric neutrino anomaly by measuring the neutrino beam at two different locations, one close to production (near detector, at Fermilab, $\sim 320 \mathrm{~m}$ from the production point), where no effect is expected from neutrino mixing, and the other far away (far detector, in the Soudan mine, Ely, Minnesota, about $730 \mathrm{~km}$ from Fermilab), where neutrino oscillations would modify the beam flavour composition and induce distortions in the measured energy spectra.

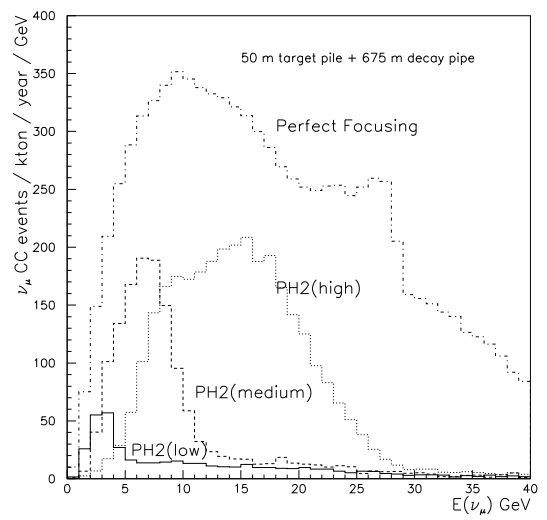

Fig. 21. Predicted event rates at the MINOS far site for the three possible beam configurations, together with the spectrum expected for "perfect focusing", where all produced neutrinos reach the detector.

The NuMI neutrino beam is obtained from the decay of secondary particles produced in the interactions of $120 \mathrm{GeV}$ protons, delivered by the new Fermilab Main Injector, with a carbon target. The expected proton flux is $4 \times 10^{13}$ protons per pulse, with repetition rate $1.9 \mathrm{~s}$ and spill duration time $10 \mu \mathrm{s}$. The secondary mesons are focused by two parabolic horns and allowed to decay in a $675 \mathrm{~m}$ long decay tunnel. The relative distance between the two magnetic elements can be changed by moving the second horn with respect to the other: by adjusting the 
horn positions and currents it is then possible to select different regions of the phase space, thus providing the flexibility to optimize the beam energy during the course of the experiment. Three beam settings are possible (see Fig.21), corresponding to three different energy regimes: low $\left(<E_{\nu}>\sim 3 \mathrm{GeV}\right)$, medium $\left(<E_{\nu}>\sim 7 \mathrm{GeV}\right)$ and high $\left(<E_{\nu}>\sim 15 \mathrm{GeV}\right)$ energy. In order to have optimal sensitivity in the low part of the atmospheric neutrinos allowed region, which seems to be preferred by Super-Kamiokande data, MINOS has decided to start taking data with the lowenergy beam configuration. Another focusing system is under consideration and might be included in the final design of the NuMI beam, the so-called hadron hose. The basic idea is to have a wire carrying a $1 \mathrm{kA}$ current down the centre of the decay tunnel, to provide additional focusing for the charged secondary mesons, with the double advantage of increasing the beam intensity and reducing the systematic uncertainties on the beam extrapolation from the near to the far site.

In order to minimize the impact of systematic uncertainties, MINOS far and near detectors ( 5.4 kton and 980 ton mass respectively) have been designed to be as similar as possible. They are both magnetised iron-scintillator sampling calorimeters with photo-multiplier readout. The average toroidal magnetic field in the iron is about $1.3 \mathrm{~T}$. The longitudinal and transverse granularity is the same for the two detectors: $2.54 \mathrm{~cm}$ thick octagonal iron plates are alternated with scintillator planes, made of plastic slabs with $1 \times 4 \mathrm{~cm}^{2}$ transverse section and lengths varying between $4 \mathrm{~m}$ and $8 \mathrm{~m}$ for the far detector planes. The slabs orientation on successive planes differs by $90^{\circ}$, so to provide two coordinate measurements on the transverse plane. A green wave-length shifting fibre, $1.2 \mathrm{~mm}$ diameter, is glued into a grove at the centre of the wide side of each slab: the light produced in the scintillator is trapped into the fibre, shifted in frequency and then routed to multi-pixel PMTs $^{l}$ through a clear fibre, optically connected to the wave-length shifting one. The readout is two-sided at the far detector and one-sided at the near detector. While the far detector planes are all fully equipped with scintillator strips, the near detector will be only partially instrumented. In the forward part (the first 120 plates, out of a total of 180), which logically defines the target and calorimeter sections, only every fifth plane is fully instrumented, while $4 / 5$ of the plates are instrumented in a limited region around the beam fiducial area. In the remaining section, the muon spectrometer, every fifth plane is fully instrumented.

A calibration detector, much smaller in size but with the same longitudinal segmentation as the MINOS far and near detectors, is being tested at the CERN PS,101 to characterize its response to muons, hadrons and electrons and to study the performances of the different hardware components used by MINOS.

The main physics goals of MINOS, which is expected to start taking data in 2004 , are to test the neutrino oscillations hypothesis, to verify that the dominant mode is $\nu_{\mu} \rightarrow \nu_{\tau}$, as suggested by Super-Kamiokande data, and to measure the

\footnotetext{
${ }^{l} 16$-pixel PMTs are used at the far detector, where the signal is optically multiplexed, while at the near detector 64-pixel PMTs have been chosen and multiplexing is applied only in the muon spectrometer section.
} 
oscillation parameters with a precision of about $10 \%$. Moreover the $\nu_{\mu} \rightarrow \nu_{s}$ and $\nu_{\mu} \rightarrow \nu_{e}$ channels will also be investigated.

The criterion envisaged by MINOS to yerify the oscillations hypothesis is the so-called T-test, based on the parameter:102

$$
T=\frac{\mathcal{N}_{1 \mu}}{\mathcal{N}_{1 \mu}+\mathcal{N}_{0 \mu}}
$$

where $\mathcal{N}_{1 \mu}$ and $\mathcal{N}_{0 \mu}$ are the number of muon-like and muon-less events respectively. If $\nu_{\mu}$ 's oscillate to another active neutrino species, say $\nu_{\tau}$ 's, the muon-like sample will be depleted (disappearance of $\nu_{\mu}$ ) and the measured $\mathcal{N}_{1 \mu}$ in the far detector will be less than that expected in the absence of oscillations; on the other hand, $\nu_{\tau} \mathrm{CC}$ events will look very much like neutral current interactions and, for neutrino energies above the $\tau$ production threshold, will populate the muon-less sample, so that the total number of events will remain unchanged. Therefore, $\nu_{\mu} \rightarrow \nu_{\tau}$ oscillations would manifest themselves as a low $T_{F A R} / T_{N E A R}$ ratio with respect to expectations in absence of oscillations. If the $\nu_{\mu}$ 's oscillated to sterile neutrinos, the muon-like and the muon-less samples would be depleted in the same manner, since sterile neutrinos do not couple with any of the weak interactions mediating bosons. Therefore for pure $\nu_{\mu} \rightarrow \nu_{s}$ oscillations the ratio $T_{F A R} / T_{N E A R}$ would be the same as for the no-oscillations case. However, the NC sample would also be affected by oscillations to sterile neutrinos, which would induce a reduction in the number of neutral current interactions and a distortion of the $\mathrm{NC}$ energy spectrum: if none of those effects is measurable, a limit can be set on the $\nu_{\mu} \rightarrow \nu_{s}$ oscillation amplitude.
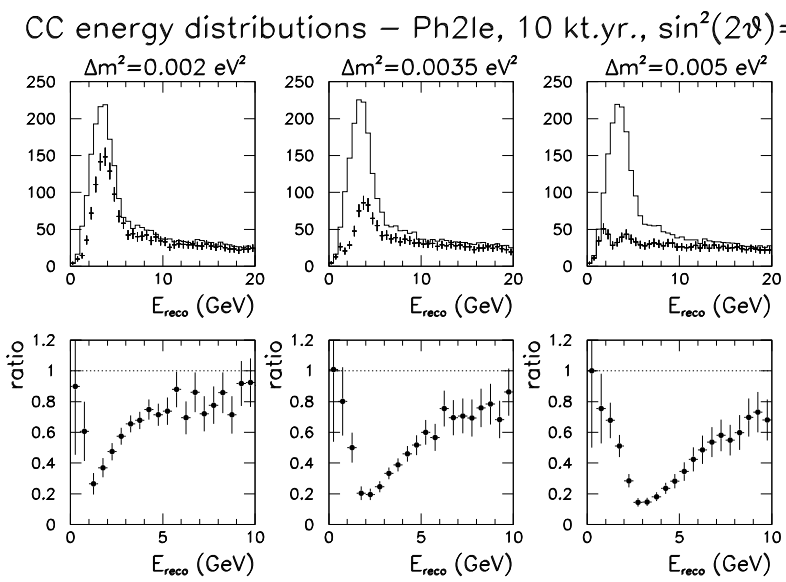

Fig. 22. (Top) Energy distributions at the MINOS far detector, in absence of oscillations (solid line) and as would be measured for different values of $\Delta m^{2}$ and $\sin ^{2} 2 \theta=0.9$. (Bottom) The ratio between the oscillated and the unoscillated spectra shows the characteristic dip shape, at an energy determined by the value of $\Delta m^{2}$ and whose depth depends on the oscillation amplitude.

If the oscillation hypothesis is confirmed by the T-test, assuming the dominant oscillation mode is $\nu_{\mu} \rightarrow \nu_{\tau}$, the oscillation parameters can be extracted by com- 
paring the $\nu_{\mu} \mathrm{CC}$ energy spectrum measured in the far detector to expectations in absence oscillations as obtained from the measured spectrum in the near detector. When considering the ratio between the two distributions (see Fig.22103), a very characteristic dip appears, at an energy corresponding to the oscillation $\Delta m^{2}$ and whose depth is determined by the oscillation amplitude $\sin ^{2} 2 \theta$.
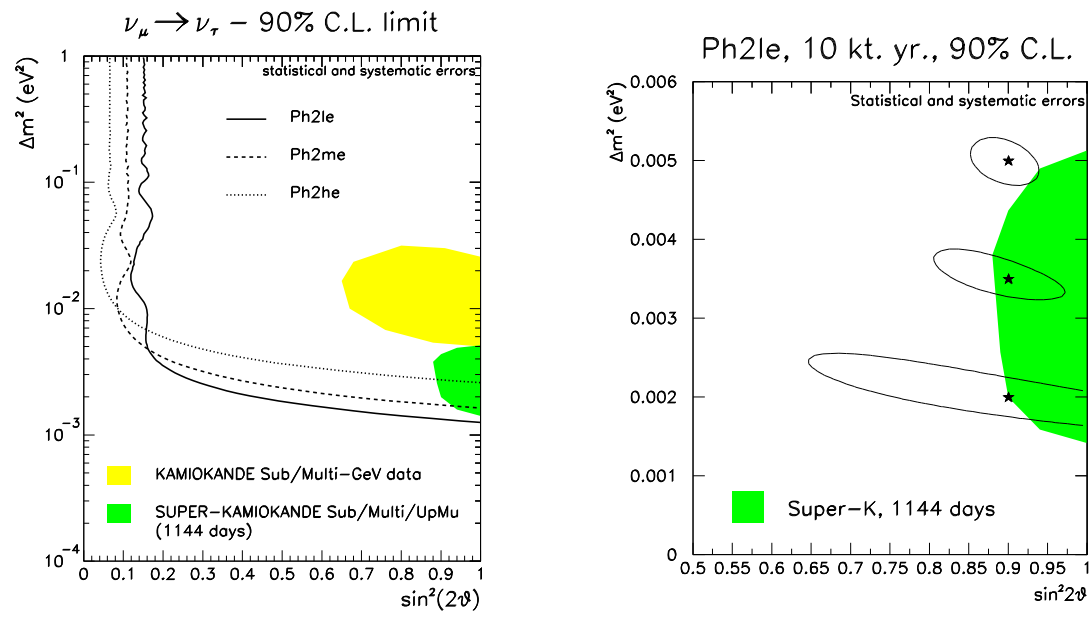

Fig. 23. (Left) MINOS sensitivity at 90\% C.L. for $\nu_{\mu} \rightarrow \nu_{\tau}$ as obtained from the T-test; results are shown for 10kton $\times$ year and all three possible beam configurations.(Right) $90 \%$ C.L. allowed regions from fits to reconstructed CC energy distribution, for $\sin ^{2} 2 \theta=0.9$ and different values of $\Delta m^{2}$; results are shown for the low-energy beam only.

If no oscillation signal is found, the $90 \%$ C.L. limits which MINOS would extract from the T-test analysis, based on 10 kton $\times$ year of data, are shown in Fig.23 (left), 103 together with the allowed regions obtained by Kamiokande and Super-Kamiokande. Using the low-energy beam, then, MINOS will cover the entire parameter space suggested by the atmospheric neutrino results. On the other hand, if an oscillation signal is observed, a measurement of the oscillation parameters can be performed. MINOS sensitivity to the oscillation parameters, as obtained from the far-to-near comparison of the $\nu_{\mu} \mathrm{CC}$ energy spectrum for $10 \mathrm{kton} \times$ year, is shown in Fig.23 (right) 103 for $\sin ^{2} 2 \theta=0.9$ and $\Delta m^{2}$ varying between $0.2 \times 10^{-3} \mathrm{eV}^{2}$ and $0.5 \times 10^{-3} e \mathrm{~V}^{2}$ : as expected, the higher the value of $\Delta m^{2}$, the better the precision to which the parameters can be determined.

MINOS also plan to study atmospheric neutrinos and preliminary results, based on a 18 kton $\times$ year sample of simulated events, show that the experiment has the sensitivity to check the atmospheric neutrino anomaly in the range of parameters suggested by Super-Kamiokande, with completely different systematics.104

Intense efforts have been recently made within the MINOS collaboration to optimize the experiment capabilities for detection of a $\nu_{\mu} \rightarrow \nu_{e}$ signal. The main 
difference with respect to the $\nu_{\mu} \rightarrow \nu_{\tau}$ search is that in case of oscillations to $\nu_{e}$ an appearance analysis has to be performed, looking for an excess of events in the far detector, above the expected background from prompt $\nu_{e} \mathrm{CC}$ interactions and $\mathrm{NC}$ events with a topology similar to that of $\nu_{e} \mathrm{CC}$. The prompt $\nu_{e}$ contamination in the $\nu_{\mu}$ beam is quite small, of the order of $0.6-2 \%$, depending on beam configuration. The systematic uncertainties on background predictions in the far detector should be drastically reduced by the measurements of the same background events in the near detector. Moreover results from hadron-production experiment should help to reduce the systematic error on beam predictions. Preliminary results 105 show that, if the total error can be kept within 10\%, MINOS should be able to improve the CHOOZ 88 limit on $\left|U_{e 3}\right|^{2}$ by about a factor two. ${ }^{m}$

The CERN-to-Gran Sasso program is based on a completely different strategy than that of NuMI-to-Soudan. Instead of measuring the disappearance of muon neutrinos at the far location and extracting the oscillation parameters from the characteristic pattern induced by oscillations in the neutrino energy distribution, they have decided to go for direct detection (appearance) of tau neutrinos in an almost pure $\nu_{\mu}$ beam. The two approaches should be regarded as complementary and equally valuable, especially in a field where redundancy is a requirement rather than a choice.

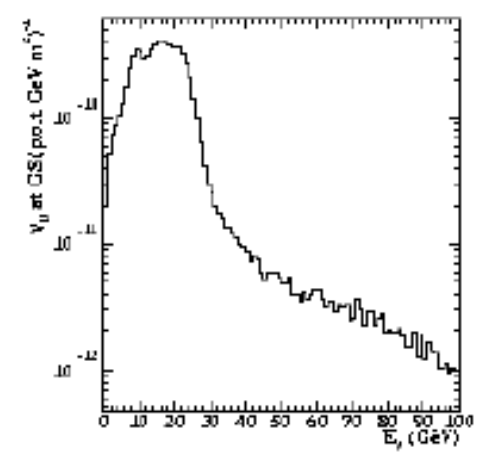

Fig. 24. CNGS $\nu_{\mu}$ flux at the Gran Sasso site.

CNGS is a wide-band neutrino beam of relatively high energy $\left(<E\left(\nu_{\mu} C C\right)>\sim\right.$ $20 \mathrm{GeV}$, see Fig.24), which has been optimised to maximize the number of $\nu_{\tau} \mathrm{CC}$ events for appearance experiments at Gran Sasso. It is expected to be commissioned by May 2005. The beam design benefits from the experience gained at CERN with the West Area Neutrino Beam (WANF), in mostrecent times used by the shortbaseline experiments NOMADL06 and CHORUS.107 Primary protons of $400 \mathrm{GeV}$ are extracted from the CERN-SPS and let interact with a graphite target, where secondary particles, mostly pions and kaons, are produced. High energy positively

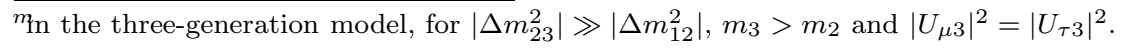


charged mesons are focused by means of two magnetic elements, the horn and the reflector, and then decay in a $900 \mathrm{~m}$ long tunnel. The expected proton intensity is of $4.5 \times 10^{19}$ pot/year. The $\bar{\nu}_{\mu}, \nu_{e}$ and $\bar{\nu}_{e}$ contamination is very low (in terms of CC interactions: $\sim 2 \%, \sim 0.8 \%$ and $0.05 \%$ respectively), while the prompt $\nu_{\tau}$ contamination $\left(O\left(10^{-6}\right)\right)$ is essentially negligible for the purposes of $\nu_{\tau}$ appearance experiments. Since the baseline is fixed (about $730 \mathrm{~km}$ between the production point at CERN and the Gran Sasso Laboratory), the low value of the $\Delta m^{2}$ suggested by atmospheric neutrino experiments would require operation at the lowest energy possible; on the other hand, neutrinos must be energetic enough to produce charged tau leptons in charged current interactions. A compromise between these two competing requirements is achieved by adjusting the settings of the beam focusing system. The expected number of $\nu_{\tau} \mathrm{CC}$ events at Gran Sasso per kton per year are given in Tab.6 (from ref.100).

Table 6. Expected number of $\nu_{\tau}$ charged current interactions for an isoscalar target fran Sasso, for $\sin ^{2} 2 \theta=1$ and different values of $\Delta m^{2}$, assuming $4.5 \times 10^{19}$ pot/year (from ref 100 ). Detector efficiencies have not been taken into account.

\begin{tabular}{ccc}
\multirow{2}{*}{$\Delta m^{2}\left(e \mathrm{~V}^{2}\right)$} & \multicolumn{2}{c}{$N_{\nu_{\tau} C C} /$ kton $/$ year } \\
\cline { 2 - 3 } & $E_{\nu}=1-30 \mathrm{GeV}$ & $E_{\nu}=1-100 \mathrm{GeV}$ \\
\hline \hline $1 \times 10^{-3}$ & 2.34 & 2.48 \\
$3 \times 10^{-3}$ & 20.7 & 21.4 \\
$5 \times 10^{-3}$ & 55.9 & 57.7 \\
$1 \times 10^{-2}$ & 195 & 202 \\
\hline
\end{tabular}

OPERA 99 is an emulsion-based experiment, which aims to detect $\nu_{\tau} \mathrm{CC}$ events by identifying the $\tau$ produced through its decay kink. Emulsions are used as high precision trackers rather than as the active target. The OPERA design, which largely benefits from the experience acquired with the CHORUS107 and DONUT10 experiments, is an evolution of the Emulsion Cloud Chamber (ECC) technique, where the high-precision tracking capabilities of emulsions are integrated with a passive material to provide a large target mass. In the OPERA baseline design, an ECC cell consists of a $1 \mathrm{~mm}$ thick lead plate followed by a thin film, made of two $50 \mu \mathrm{m}$ thick emulsion layers separated by a $200 \mu \mathrm{m}$ plastic base. The basic detector unit is obtained by stacking 56 cells to form a compact brick $\left(10.2 \times 12.7 \times \mathrm{cm}^{2}\right.$ transverse section, $10 X_{0}$ length, $8.3 \mathrm{~kg}$ weight), which can in turn be assembled into walls. An additional emulsion film is placed in front of the first lead plate, to improve the track matching with the upstream wall, while another film, the socalled Special Sheet, separated from the most downstream cell by a $2 \mathrm{~mm}$ plastic plate, is used to have good $\tau$ detection efficiency also for events occurring in the last lead plate. After each wall, an electronic tracking detector is used to select the brick where the neutrino interaction occurs. In the baseline design the tracking detector in the target section follows very closely that of MINOS, with scintillator strips coupled to wave-length shifting fibres and multi-anode PMT readout, where the selected PMTs are the same as those used in MINOS near detector. A wall of bricks and two planes of electronic detectors constitute one OPERA module, 
whose structure is then repeated 24 times to form a supermodule, also including a downstream muon spectrometer. Each muon spectrometer consists of a dipolar magnet (1.55 $\mathrm{T}$ in the tracking region), made of two vertical walls of iron layers, interleaved with RPC detectors, and of drift tubes, placed in front and behind the magnet as well as between the two walls. Three supermodules form the full OPERA detector, for a total target mass of about 1.8 kton.

The data from the electronic detectors, which will be analysed quasi-online, are used to select bricks where neutrino interactions occur and which have to be removed by an automated system. Additional bricks might need to be removed for a complete reconstruction of the interesting events. Events selected as $\tau$ candidates will then be sent to dedicated scanning stations, where further studies can be performed to achieve the required background suppression. The expected rate of $\nu_{\mu} \mathrm{CC}$ events is of about 30 events per day.

The $\tau$ decay channels considered by OPERA are listed in Tab.7, together with the corresponding $\nu_{\tau}$ detection efficiencies and the expected number of background events ${ }^{n}$ per year. New studies are underway to improve the experiment sensitivity by adding also the $\tau \rightarrow \rho \nu$ channel (23.5\% B.R.) to the decay modes considered. The expected number of $\tau$ events per year is given in Tab.8 as a function of possible values of $\Delta m^{2}$.

Table 7. OPERA efficiency for the $\tau$ decay channels considered and the corresponding expected number of background events per year. The efficiencies, which also include the branching ratio for each decay channel, have been obtained as weighted sums of the efficiencies for DIS and non-scaling processes.

\begin{tabular}{ccc} 
Decay mode & $\epsilon\left(\nu_{\tau} C C\right)(\%)$ & $N_{B K G D} /$ year \\
\hline \hline$\tau \rightarrow e$ & 3.7 & 0.04 \\
$\tau \rightarrow \mu$ & 2.7 & 0.03 \\
$\tau \rightarrow h$ & 2.3 & 0.05 \\
\hline
\end{tabular}

Table 8. Expected num of $\tau$ events observed in OPERA per year as a function of possible values of $\Delta m^{2}$ (from ref.108).

\begin{tabular}{cc}
$\Delta m^{2}\left(\mathrm{eV}^{2}\right)$ & $N_{\tau} /$ year \\
\hline \hline $1.5 \times 10^{-3}$ & 0.82 \\
$2.5 \times 10^{-3}$ & 2.82 \\
$3.2 \times 10^{-3}$ & 3.66 \\
\hline
\end{tabular}

If no signal is observed, the average upper limit at 90\% C.L. which would be obtained by OPERA is shown in Fig 25 (left) for 2 and 5 years of exposure respectively. On the other hand, if $\nu_{\tau}$ events are actually observed, a measurement of $\Delta m^{2}$ can be performed. Assuming maximal mixing and $\Delta m^{2}=3.2 \times 10^{-3} \mathrm{eV}^{2}$, the $90 \%$ C.L. allowed region for the oscillation parameters as determined by OPERA after 5 years of data taking is shown in Fig.25 (right).

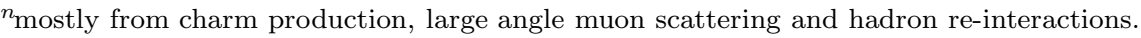



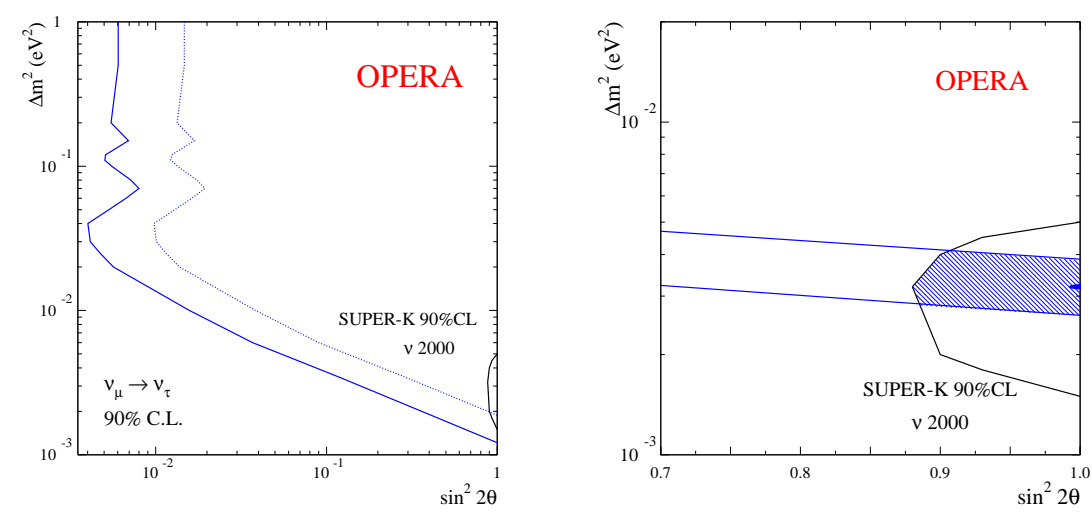

Fig. 25. (Left) Average upper limit at $90 \%$ C.L. which would be obtained by OPERA if no oscillation signal is detected, after 2 and 5 years of data-taking. (Right) 90\% C.L. allowed region for the oscillation parameters as determined by OPERA after 5 years of running.

OPERA also plan to perform a $\nu_{\mu} \rightarrow \nu_{e}$ oscillation analysis, by looking for $\nu_{e}$ appearance above the $\sim 1 \%$ intrinsic $\nu_{e}$ contamination, and to search for neutrino oscillations by studying the ratio between $\mathrm{NC}$ and $\mathrm{CC}$ interactions. 99 However the study of the systematic uncertainties for those analyses is likely to be somehow limited by the lack of a near detector.

The other experiment which is being planned at the Gran Sasso laboratories to detect neutrinos from NCGS is based on the ICARUS43,109 technology. ICARUS is a liquid-Argon TPC which allows event reconstruction in three dimensions, combining high spatial resolution with excellent particle identification capabilities and precise homogeneous calorimetry. ICARUS has been conceived as a multi-purpose experiment with a very wide physics program, which goes from proton decay searches to the study of solar and atmospheric neutrinos as well as of supernova neutrinos and, of course, of artificially produced neutrinos from CERN.

The final phase of ICARUS foresees a detector with a sensitive mass of at least 5 kton, but a step-wise strategy has been adopted to demonstrate the technical feasibility of such a challenging project. The first large-scale prototype had a volume of $1,000 \mathrm{~m}^{3}$ (15 ton) and was built as a test-bench for the cryogenic system, the internal detector mechanics and the liquid-Argon purification. The 15 ton prototype was successfully tested between 1997 and 1999. The next step towards the fullscale detector is the T600 module 110 which is now being commissioned at Pavia and which will be transported to the Gran Sasso Laboratory tunnel as soon as the technical tests are complete. In 1999 a proposal was submitted for the ICANOE experiment,100 which combined the ICARUS technology with a fine-grained iron calorimeter developed by the NOE collaboration.111

Because of the high resolution on the event kinematic variables, ICANOE could search for $\nu_{\tau}$ appearance in the NCGS beam using kinematic selection criteria, 


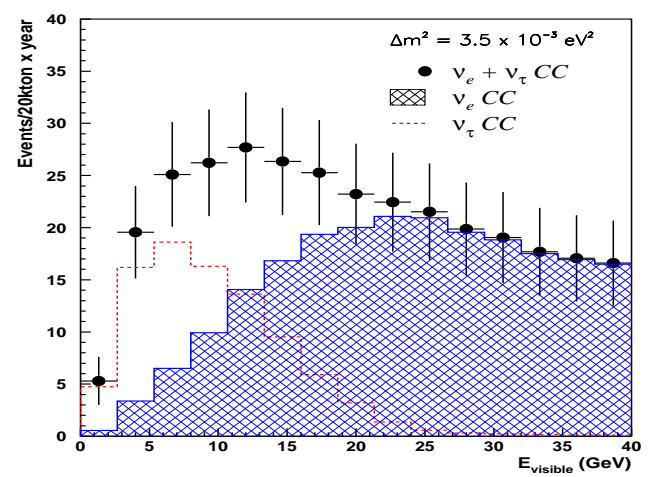

Fig. 26. ICANOE expected visible energy distribution for events with a primary electron, in the cas $-\nu_{\mu} \rightarrow \nu_{\tau}$ oscillations with maximum mixing angle and $\Delta m^{2}=3.5 \times 10^{-3} \mathrm{eV}^{2}$ (from ref.113). Both the background from the prompt $\nu_{e}$ and $\bar{\nu}_{e}$ in the beam (hatched histogram) and the events from $\tau \rightarrow e$ decays (dashed line) have been considered. Errors are only statistical.

a strategy which has been successfully applied by the short-baseline experiment NOMAD.112 For ICANOE, as for NOMAD, the $\tau \rightarrow e$ decay mode represents a golden channel. After four years of running with intensity $4.5 \times 10^{19} \mathrm{pot} /$ year, the expected number of $\nu_{\tau} \mathrm{CC}$ events followed by a $\tau \rightarrow e \nu \bar{\nu}$ decay would be about 110 for $\Delta m^{2}=3.5 \times 10^{-3} \mathrm{eV}^{2}$, while the total expected number of background events from prompt $\nu_{e}$ and $\bar{\nu}_{e}$ is about 470. This means that an excess of events should be measured in the visible energy distribution even before applying any kinematic cut (see Fig.26).

The excellent electron identification in ICANOE would also allow a search for $\nu_{e}$ appearance from $\nu_{\mu} \rightarrow \nu_{e}$ oscillations. Selection criteria based on kinematic variables could be used to discriminate between events from $\nu_{\mu} \rightarrow \nu_{e}$ and events from $\tau \rightarrow e$ decays. Assuming $\Delta m_{23}^{2}=3.5 \times 10^{-3} \mathrm{eV}^{2}$ and $\theta_{23}=45^{0}$, the statistical significance for a $\nu_{\mu} \rightarrow \nu_{e}$ signal (after four years of running with intensity $4.5 \times$ $10^{19} \mathrm{pot} /$ year) varies between $0.8 \sigma$ and $6.8 \sigma$ for $3^{0}<\theta_{13}<9^{0} .113$

In summer 2000 the ICANOE collaboration ceased to exist as such, but it is likely that as soon as the successful operation of the T600 module is demonstrated, an updated version of the proposal will be submitted to the relevant organisations. 114

Until now we have discussed the experimental hints for neutrino oscillations coming from the study of naturally produced neutrinos and we have described the experimental program which, in the near future, should clarify many of the open issues concerning the solar and the atmospheric neutrino anomalies. There is a third hint for neutrino oscillations, which has the peculiarity of being the only one coming from an accelerator experiment and the only one to represent evidence for neutrino oscillations in the appearance mode. This is the so-called LSND effect, which we shall discuss in the next section. 


\section{The LSND Effect}

The LSND experiment115 at the Los Alamos Meson Physics Facility (LAMPF), New Mexico, USA, was originally designed to search for $\bar{\nu}_{\mu} \rightarrow \bar{\nu}_{e}$ oscillations using $\bar{\nu}_{\mu}$ from $\mu^{+}$decay at rest (DAR). In 1995 LSND reported an excess of electronlike events above background and gave an interpretation of that result in terms of neutrino oscillations.116 The effect was later confirmed by an improved analysis with larger statistics, both in DAR $\bar{\nu}_{\mu}$ 's 117 and in the $\nu_{\mu}$ sample from $\pi^{+}$decay in flight (DIF). 118 In the following we shall discuss the results reported in a recent paper of the LSND collaboration, 119 where DAR and DIF events are treated in the same way, in a unified analysis of the two samples.

The neutrino beam used by LSND was obtained from the decay of the secondary particles, mostly $\pi^{+}$with a small fraction of $\pi^{-}\left(N_{\pi^{-}} / N_{\pi^{+}} \sim 1 / 8\right)$, produced in the interaction of an intense $(\sim 1 \mathrm{~mA}) 798 \mathrm{MeV}$ proton beam with the primary target.

The decay chains leading to the production of neutrinos and anti-neutrinos are:

$$
\begin{aligned}
\pi^{+} \rightarrow & \mu^{+} \nu_{\mu} \\
& \hookrightarrow e^{+} \nu_{e} \bar{\nu}_{\mu}
\end{aligned}
$$

and the charge-symmetric sequence:

$$
\begin{aligned}
\pi^{-} \rightarrow \mu^{-} \bar{\nu}_{\mu} & \\
& \hookrightarrow e^{-} \bar{\nu}_{e} \nu_{\mu} .
\end{aligned}
$$

The fractions of $\pi^{+}$DAR and DIF are $96.6 \%$ and $3.4 \%$ of the total respectively. While only $\sim 0.05 \%$ of the positive muons produced in (50) decay in flight, most of them stop before decaying and then give a standard Michel spectrum for $\nu_{e}$ and $\bar{\nu}_{\mu}$ $\left(E_{\max }\left(\bar{\nu}_{\mu}\right)=m_{\mu} / 2=52.8 \mathrm{MeV}\right)$. About $95 \%$ of the $\pi^{-}$'s are stopped in the target and immediately captured, while the remaining $5 \%$ decay in flight; moreover, about $88 \%$ of the muons from the $\pi^{-}$DIF are captured from atomic orbit. In conclusion a suppression of the order of $\sim 1 / 8 \times 0.05 \times(1-0.88)=7.5 \times 10^{-4}$ is expected for the relative flux of prompt $\bar{\nu}_{e}$ 's, which constitute a background for the $\bar{\nu}_{\mu} \rightarrow \bar{\nu}_{e}$ oscillation search. The $\nu_{e}$ flux from $\mu^{+}$and $\pi^{+}$DIF, representing a background for the $\nu_{\mu} \rightarrow \nu_{e}$ oscillation search, is suppressed by the long muon lifetime and by the small $\pi^{+} \rightarrow e^{+} \nu_{e}$ branching ratio $\left(1.23 \times 10^{-4}\right)$.

In 1996 the production target, which was made of water during the running period 1993-1995, was upgraded: two upstream targets, used to provide beam to other experiments, were removed and the water production target was replaced by tungsten. This resulted in a reduction of the DAR flux per pot and of the DIF flux per pot by $27 \%$ and $34 \%$ respectively.

The estimated systematic uncertainty on the beam predictions is $7 \%$ for $\mu^{+}$ fluxes and $15 \%$ for $\pi^{ \pm}$DIF and $\mu^{-}$DAR fluxes.

The LSND detector was an approximately cylindrical tank $(8.3 \mathrm{~m}$ long, $5.7 \mathrm{~m}$ diameter), located $30 \mathrm{~m}$ downstream from the neutrino source (sensitivity to $\Delta m^{2} \sim$ $\left.0.1-1 e \mathrm{~V}^{2}\right)$ and filled with 167 tons of mineral oil doped with scintillator $(0.031 \mathrm{~g} /$ liter 
of butyl-PBD). Because of the low scintillator concentration, both scintillation and Cherenkov light could be detected by the 1220 photo-multiplier installed on the inside surface of the tank (25\% coverage). The Cherenkov cone and the time distribution of the light were used to tag electron events and to measure the event vertex and the $e^{ \pm}$direction. For any PMT activity above threshold, the pulse height, a digitised time and the event position were recorded, so that energy measurements and space and time correlation were possible. On all sides except the bottom the detector was surrounded by an active shield (scintillator plus lead), providing a veto system against charged particles $\left(10^{-5}\right.$ inefficiency). Triggers were not required to be in-spill, but the beam state was recorded by the data acquisition and a beamon/beam-off comparison was used to perform a statistical subtraction of the residual cosmic ray background.

The identification of $\bar{\nu}_{e}$ events in LSND is based on the detection of quasi-elastic scattering:

$$
\bar{\nu}_{e}+p \rightarrow e^{+}+n
$$

followed by the neutron capture process:

$$
n+p \rightarrow d+\gamma(2.2 \mathrm{MeV})
$$

Therefore the $\bar{\nu}_{e}$ signature in LSND is a delayed coincidence between an electron signal and a spatially correlated $2.2 \mathrm{MeV}$ photon.

Electron neutrinos are identified in LSND via the inclusive charged-current reaction:

$$
\nu_{e} C \rightarrow e^{-} X
$$

Although the primary electron selection criteria have been optimize for the DAR analysis, the same cuts are used to select also electrons for the DIF analysis. The electron energy range defined for the $\bar{\nu}_{\mu} \rightarrow \bar{\nu}_{e}$ search is $20<E_{e}<60 \mathrm{MeV}$, while events with $60<E_{e}<200 \mathrm{MeV}$ are used for the $\nu_{\mu} \rightarrow \nu_{e}$ analysis. The cut at $20 \mathrm{MeV}$ rejects the background events from the $\beta$-decay of ${ }^{12} B$ produced in the capture of cosmic $\mu^{-}$on ${ }^{12} C$, while events above $200 \mathrm{MeV}$ are removed to suppress the beam-related background from $\pi^{+} \rightarrow e^{+} \nu_{e}$, which at those energies becomes larger that any possible oscillation signal. Cosmic ray events are further suppressed by using the veto information and by removing electron events with additional activity within $8 \mu \mathrm{s}$ before the electron signal or $12 \mu \mathrm{s}$ after it. Discrimination between correlated $2.2 \mathrm{MeV}$ photons from neutron capture and accidental $\gamma$ 's from radioactivity is achieved by means of a likelihood function, $R_{\gamma}$, which depends on the number of PMT hits, the distance between the reconstructed $\gamma$ and $e^{+}$positions and the time interval between the $\gamma$ and the $e^{+}$events. The discrimination power of $R_{\gamma}$ has been checked by using $\nu_{e} C \rightarrow e^{-} N_{g . s}$. exclusive interactions, where no recoil neutron is present and which should therefore exhibit an $R_{\gamma}$ distribution consistent with that expected for purely accidental $\gamma$ 's. 
The two largest backgrounds to the $\bar{\nu}_{\mu} \rightarrow \bar{\nu}_{e}$ signal are $\bar{\nu}_{e} \mathrm{CC}$ interactions from prompt $\bar{\nu}_{e}$ in the beam, which are identical to the signal events, or $\nu_{\mu}\left(\bar{\nu}_{\mu}\right) \mathrm{CC}$ interactions with a correlated neutron signal and the muon misidentified as a positron. Both backgrounds have been estimated by using the experiment Monte Carlo simulation and, whenever possible, by means of control samples from real data.

For the entire period 1993-1998 of data-taking, LSND observed a beam on-off excess of $117.9 \pm 22.4$ events in the DAR channel. The corresponding expected number of background events from $\mu^{-}$DAR followed by $\bar{\nu}_{e} p \rightarrow e^{+} n$ scattering and from $\pi^{-}$DIF followed by $\bar{\nu}_{\mu} p \rightarrow \mu^{+} n$ scattering are $19.5 \pm 3.9$ and $10.5 \pm 4.6$ respectively leading to a total excess of $87.9 \pm 22.4 \pm 6.0$ events above background expectations 110 The number of expected $\bar{\nu}_{e}$ events from $\bar{\nu}_{\mu} \rightarrow \bar{\nu}_{e}$ oscillations with $100 \%$ probability is $33000 \pm 3300$, the error being a combination of the systematic uncertainties on the neutrino flux $(7 \%)$ and on the $e^{+}$efficiency $(7 \%)$. The result then corresponds to an oscillation probability of $(0.264 \pm 0.067$ (stat. $) \pm 0.045($ sys. $)) \%, 119$ to be compared with the previously published result of $(0.31 \pm 0.12$ (stat. $) \pm 0.05($ sys. $)) \%, 117$ which was based on the 1993-1995 data sample.

The new DIF $\nu_{\mu} \rightarrow \nu_{e}$ oscillation analysis gives a beam on-off excess of $14.7 \pm 12.2$ events. The estimated total background from the $\mu^{+} \rightarrow e^{+} \nu_{e} \bar{\nu}_{\mu}, \pi^{+} \rightarrow e^{+}$and $\nu e \rightarrow \nu e$ processes is of $6.6 \pm 1.7 \nu_{e}$ events, resulting in a total excess of $8.1 \pm 12.2 \pm 1.7$ events above background expectations. The number of events expected for $\nu_{\mu} \rightarrow \nu_{e}$ oscillation with $100 \%$ probability is 7800 , thus giving an oscillation probability of $(0.10 \pm 0.16 \pm 0.04) \% .119$ This has to be compared with the result obtained from the 1993-1995 data analysis, which gave a probability of $(0.26 \pm 0.10 \pm 0.05) \% 118$ Using the result from the DAR analysis and assuming that $\mathrm{CP}$ is conserved, the expected oscillation probability in the DIF sample is $\sim 0.26 \%$ at high $\Delta m^{2}$ and $\sim 0.05 \%$ at low $\Delta m^{2}$

A likelihood fit of the beam-on events which pass their oscillation cuts is used to extract the LSND allowed region in the $\sin ^{2} 2 \theta, \Delta m^{2}$ plane. The fit is performed over the entire electron range $20-200 \mathrm{MeV}$, so that both DAR and DIF events are considered at the same time. The probability density functions which define the likelihood function $\mathcal{L}$ used in the fit depend on the electron energy, the electron longitudinal position along the tank, the cosine of the reconstructed angle between the neutrino and the electron direction, the likelihood ratio $R_{\gamma}$ for correlated $\gamma$ selection. To account for the limited knowledge of the background, its contribution to $\mathcal{L}$ is allowed to vary with gaussian profile around the expected central value. ${ }^{o}$

The result obtained is shown in Fig.27, where the LSND allowed region is compared with limits obtained by other experiments sensitive to $\nu_{\mu} \rightarrow \nu_{e}\left(\bar{\nu}_{\mu} \rightarrow \bar{\nu}_{e}\right)$ oscillations in different regions of the parameter space.120.121. 122.123 In particular it is interesting to briefly discuss the null result obtained by the KARMEN2 collaboration, which has conducted a search for $\bar{\nu}_{\mu} \rightarrow \bar{\nu}_{e}$ oscillations in approximately the same region of the parameter space as that investigated by LSND.

obeam-related background are locked to vary together, while beam-unrelated backgrounds can fluctuate independently. 


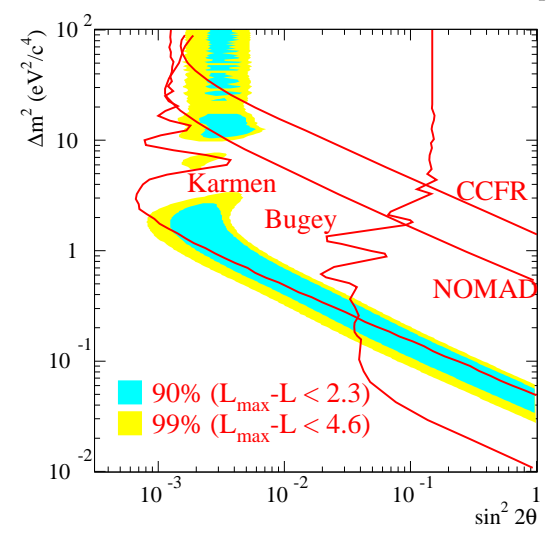

Fig. 27. Allowed obtained by LSND from a combined fit of the $\bar{\nu}_{\mu} \rightarrow \bar{\nu}_{e}$ and $\nu_{\mu} \rightarrow \nu_{e}$ samples (from ref. 119 ; blue is $90 \%$ C.L., yellow is $99 \%$ C.L.).

The KARMEN2 experiment was performed at the ISIS neutron spallation facility at the Rutherford Appleton Laboratory, Chilton, Didcot, United Kingdom. As at LAMPF, neutrinos are obtained from the decay at rest or in flight of secondary particles produced in the interactions of $800 \mathrm{MeV}$ primary protons with a massive beam stop target. Also in this case the $\nu_{\mu}$ 's from $\pi^{+}$decays are monoenergetic $\left(E_{\nu_{\mu}}=29.8 \mathrm{MeV}\right)$, while both $\nu_{e}$ 's and $\bar{\nu}_{\mu}$ 's have continuous energy spectra extending up to $52.8 \mathrm{MeV}$. One difference between the two beams is that the one at ISIS has a well defined time structure: two very short $(\sim 100 \mathrm{~ns})$ proton pulses, separated by $225 \mathrm{~ns}$, are produced with a repetition rate of $50 \mathrm{~Hz}$. Because of the different lifetimes of their parent particles $\left(\tau_{\pi}=26 \mathrm{~ns}, \tau_{\mu}=2.2 \mu \mathrm{s}\right)$, the $\nu_{\mu}$-burst, which occurs essentially in coincidence with the proton pulses, can be clearly separated from the $\nu_{e^{-}}$and $\bar{\nu}_{\mu}$-induced events, which are instead characterised by a much longer decay constant. Due to the accelerator duty cycle of $10^{-5}$ (to be compared with the $6 \times 10^{-2}$ duty factor for LSND), the cosmic ray background is largely suppressed with respect to the beam-related events. The proton beam intensity at ISIS is $0.2 \mathrm{~mA}$, that is to say a factor of five lower than at LAMPF.

The KARMEN detector,224 located $17.5 \mathrm{~m}$ downstream the neutrino source (about half the LSND baseline), consisted of a rectangular tank filled with $56 \mathrm{t}$ liquid scintillator. The tank was segmented into 512 optically independent modules, wrapped with $\mathrm{Gd}_{2} \mathrm{O}_{3}$ coated paper to increase the efficiency for the neutron capture process. The event position was obtained from the time of the PMT signal at the two ends of the hit module. Shielding against the beam-related neutron background and the cosmic ray background was provided by a $7 \mathrm{kton}$ steel blockhouse combined with a system of two layers of veto counters. Moreover an additional veto counter, added in 1996 for the KARMEN2 upgrade, was used to largely suppress the background from energetic neutrons produced in $\nu_{\mu}$ CC DIS interactions.

The $\bar{\nu}_{e}$ detection in KARMEN2 was based on the delayed coincidence between the positron signal from reaction (54) and either the $2.2 \mathrm{MeV}$ photon of reaction 
(55) or the $8 \mathrm{MeV}$ integrated photon signal from Gd de-excitation following neutron capture on gadolinium. No particle identification was performed and the tagging of the positron event, which was expected within $2.2 \mu \mathrm{s}$ after the beam-on-target signal, relied on the beam time structure alone. Both time and spatial correlation between the positron and the neutron capture events were required. An analysis of the data collected between February 1997 and March 2000 shows that 11 events have been identified as $\bar{\nu}_{e}$ interactions, with an expected background ${ }^{p}$ of $12.3 \pm 0.6$ events. 122 Therefore the KARMEN2 result is compatible with the no-oscillation hypothesis and the corresponding exclusion plot at 90\% C.L. is shown in Fig.27.

Combining the LSND result with those obtained by all the other experiments which, having studied the same transition, have obtained null results, only a small portion of the LSND solutions is still allowed to explain the experiment anomaly in terms of neutrino oscillation. In particular, the skeptical reader may find the LSND and KARMEN2 results quite contradictory. However it has to be stressed that the two experiments have sensitivities which peak at slightly different values of $\Delta m^{2}$. Moreover, before any definitive conclusion can be drawn from the various results, a combined analysis of all experimental data should be performed. This has been tried for LSND and KARMEN, but only on subset of the collected data 125 In conclusion it is fair to say that no definitive answer has yet been given to the LSND puzzle and that more experimental data is needed.

The MiniBOONE experiment, 226 which is now being built at Fermilab, will address the LSND effect by searching for $\nu_{\mu} \rightarrow \nu_{e}$ oscillations in the same region of parameter space. An almost pure $\nu_{\mu}$ beam $\left(\nu_{e}\right.$ contamination $\left.<0.3 \%\right)$, with energy in the range $0.5-1 \mathrm{GeV}$, will be obtained from the decay of secondary particles produced in the interactions of $8 \mathrm{GeV}$ protons with a beryllium target positioned in a magnetic horn. The protons will be delivered by the Fermilab Booster, with an expected intensity of $5 \times 10^{20}$ pot per year. The decay length for the positively charged secondary mesons, which are focused by the single horn, can be changed from $50 \mathrm{~m}$ to $25 \mathrm{~m}$ by inserting an intermediate absorber inside the decay tunnel, thus allowing a systematic study of the prompt $\nu_{e}$ contamination of the beam. The MiniBOONE detector will be located about $500 \mathrm{~m}$ from the neutrino production point, so that the $E / L$ ratio will be approximately the same as LSND's. MiniBOONE will consists of a spherical tank (12.2 m diameter) filled with 800 tons of pure mineral oil. The Cherenkov light produced in neutrino interactions in the inner part of the detector (445 ton fiducial volume), as well as the modest amount of scintillation light from intrinsic impurities in the oil, will be detected by 1280 PMTs (10\% coverage) installed on a support structure in the tank. This structure will also serve as an optical insulator for the inner part of the detector, while the outer $35 \mathrm{~cm}$ thick oil layer, read out by 280 additional PMTs, can be used as a veto system.

The main background components will be due to the $\nu_{e}$ contamination in the

${ }^{p}$ Background events come from cosmic muon interactions, $\nu_{e}^{12} C \rightarrow e^{-12} N_{g . s}$. sequences, prompt $\bar{\nu}_{e}$ contamination in the beam and neutrino-induced accidental coincidences. 
beam and to the misidentification of muons and $\pi^{0}$ as electrons. ${ }^{q}$ The estimated systematic uncertainties on the $\nu_{e}$ flux is about $5 \%$ for the neutrino component originating from the $\pi^{+} \rightarrow \mu^{+} \rightarrow \nu_{e}$ chain and $10 \%$ for $\nu_{e}$ 's coming from $K_{e 3}$ decays of secondary $K^{+}$and $K_{L}^{0}$. Particle identification will be performed using the sharpness of the Cherenkov rings and the ratio between the amount of prompt (Cherenkov) and late (scintillation) light, which is different for electrons and muons. The misidentification uncertainty is expected to be less than $5 \%$ for muons and about $5 \%$ for neutral pions: in the case of muons, the the systematic error can be estimated using a sample of muon decays from real data, while for $\pi^{0}$ 's it will be obtained from a Monte Carlo simulation, which can in turn be constrained using reconstructed $\pi^{0}$ 's from real data.

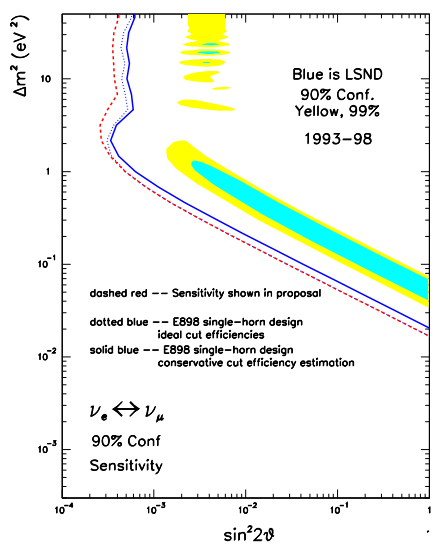

Fig. 28. Final 90\% C.L. expected sensitivity for MiniBOONE (figure from ref.127). Different beam configurations and cut efficiencies have been considered.

If oscillations occur with parameters compatible with LSND solutions, after one year of running MiniBOONE should observe an excess of $\sim 1500 \nu_{e}$ events above background expectations, with a significance of $\sim 8-10 \sigma$. On the other hand, in case of null result, after two years of running MiniBOONE should be able to completely exclude the entire LSND allowed region at 90\% C.L. (see Fig 28).

\section{Neutrino Masses and Global Analysis of Neutrino Oscillation Data}

If we believe that all experimental hints on neutrino oscillations are correct and we accept that they are all manifestations of flavour mixing in the lepton sector, we are forced to introduce at least another neutrino species in our framework. In fact, the $\Delta m^{2}$ regimes suggested by the the solar neutrino deficit, the atmospheric neutrino anomaly and the LSND effect are all well disconnected one from the other:

$$
\Delta m_{\odot}^{2} \ll \Delta m_{\text {atm }}^{2} \ll \Delta m_{L S N D}^{2} .
$$

$\bar{q}$ Given the higher neutrino energy with respect to LSND, neutrons will not constitute a significant background for MiniBOONE 
On the other hand, only two independent values of $\Delta m^{2}$ are possible for three different neutrino masses $\left(\Delta m_{31}^{2}=\Delta m_{22}^{2}+\Delta m_{21}^{2}\right)$. Therefore, recalling the LEP result on the number of active neutrinos, 6 in order to accommodate all experimental results, an additional sterile neutrino would be required.

While both the solar and the atmospheric neutrino deficit have been confirmed by more than one experiment, using different experimental techniques, no other experiment has confirmed LSND observations. For this reason, given also the enormous impact that accepting the LSND result would have on our understanding of sub-nuclear phenomena, with the introduction of a light sterile neutrino, there is a tendency in the scientific community to adopt a "conservative" attitude and to exclude LSND from most phenomenogical models and fits to neutrino oscillation parameters, until more solid experimental data become available. In the following we shall take the same approach and discuss some of the results obtained from threeneutrino analyses of the neutrino oscillation data, while only briefly commenting on the possibility to have a fourth neutrino.

Neglecting possible CP violation phases, which are anyhow not accessible to present experiments, the lepton mixing matrix 13 depends only on five parameters, two $\Delta m^{2}$ and three mixing angles, four of which can be identified with the parameters measured by solar and atmospheric experiments:

$$
\begin{aligned}
\Delta m_{\odot}^{2} & \equiv \Delta m_{21}^{2} \\
\Delta m_{a t m}^{2} & \equiv \Delta m_{32}^{2} \\
\theta_{\odot} & \equiv \theta_{12} \\
\theta_{a t m} & \equiv \theta_{23}
\end{aligned}
$$

while a limit on the fifth, $\theta_{13}$, is set by the $\mathrm{CHOOZ}$ reactor experiment.

The hierarchy $\Delta m_{\odot}^{2} \ll \Delta m_{a t m}^{2}$ implies that one of the three mass eigenstates, say $\nu_{3}$, is separated by a larger gap $\left|\Delta m_{31}^{2}\right| \approx\left|\Delta m_{32}^{2}\right| \approx \Delta m_{\text {atm }}^{2} \sim 10^{-3} \mathrm{eV}^{2}$ from the other two, $\nu_{1}$ and $\nu_{2}$, which are instead quasi-degenerate, being separated by $\left|\Delta m_{21}^{2}\right| \approx \Delta m_{\odot}^{2} \lesssim 10^{-4} \mathrm{eV}^{2}$. However, since oscillations determine only mass splittings and not the absolute value of the masses, it is still possible to shift the absolute mass scale without affecting the oscillation phenomenology. Although different mass orderings are possible, here we consider the normal hierarchical scheme, corresponding to:

$$
m_{1}<m_{2} \ll m_{3}
$$

and thus:

$$
\Delta m_{21}^{2} \ll \Delta m_{32}^{2} \approx \Delta m_{31}^{2}
$$

It can be shown that, given the strong hierarchical constraint $\Delta m_{\odot}^{2} \ll \Delta m_{a t m}^{2}$, the solar neutrino data can be described in terms of only three parameters, $\Delta m_{21}^{2}$, $\theta_{12}$ and $\theta_{13}$, while the atmospheric neutrino data can be analysed in terms of $\Delta m_{32}^{2}$, 
$\theta_{23}$ and $\theta_{13}$. For the CHOOZ data, the survival probability depends only on $\theta_{13}$ and $\Delta m_{32}^{2}$. Thus $\theta_{13}$ is the only parameter common to the three data sets. In particular, in the limit $\theta_{13}=0$ atmospheric and solar neutrino oscillation can be described by two independent two-flavour oscillation analyses.

Severalanalyses of the available data have been performed in the three-neutrino scheme 90,28 in the following we shall refer to the results presented in ref. 20 . In particular it will be interesting to see how the inclusion of different data in the fit affects the constraint which can be extracted on the mixing angle $\theta_{13}$.

The three-dimensional analysis of ref.90 of the solar neutrino data has shown that solutions with small values of the mixing angle $\theta_{13}$ are preferred, the most favourable scenario being that of two-flavour mixing $\left(\theta_{13}=0\right)$. The upper limit which can be extracted on the mixing angle based on solar neutrino data only is $\tan ^{2} \theta_{13}<2.4\left(\theta_{13}<57^{0}\right)$ at $90 \%$ C.L..

If only atmospheric neutrinos are considered, the analysis favours the $\nu_{\mu} \rightarrow \nu_{\tau}$ oscillation hypothesis. Also in this case the best fit corresponds to a small value of the mixing angle $\theta_{13}\left(\theta_{13}=9^{0}\right)$. However oscillations between muon and electron neutrinos are still allowed as a sub-dominant mode, with the following $90 \%$ C.L. allowed ranges for the relevant oscillation parameters:

$$
\begin{gathered}
1.6 \times 10^{-3} e^{2}<\Delta m_{32}^{2}<6 \times 10^{-3} e^{2} \\
0.43<\tan ^{2} \theta_{23}<4.2 \\
\tan ^{2} \theta_{13}<0.34
\end{gathered}
$$

If the $\mathrm{CHOOZ}$ result is included in the atmospheric neutrino analysis, because the full allowed region of $\Delta m_{32}^{2}$ lies within the parameter domain accessible to CHOOZ, a tighter constraint can be obtained on the mixing angle $\theta_{13}$ and the corresponding $90 \%$ C.L. limit becomes even more stringent: $\tan ^{2} \theta_{13}<0.043\left(\theta_{13}<\right.$ $\left.12^{0}\right)$.

Finally, a combined analysis of solar, atmospheric and reactor data gives, at $90 \%$ C.L., the following allowed ranges for $\Delta m_{32}^{2}, \tan ^{2} \theta_{23}$ and $\tan ^{2} \theta_{13}$, where the limits on $\tan ^{2} \theta_{13}$ are given for the SMA solution of the solar neutrino deficit as well as for the LMA or unconstrained case: ${ }^{r}$

$$
\begin{array}{rlr}
1.4 \times 10^{-3} \mathrm{eV}^{2}<\Delta m_{32}^{2} & <6 \times 10^{-3} \mathrm{eV}^{2} & \\
0.39< & \tan ^{2} \theta_{23}<3.1 & \\
\tan ^{2} \theta_{13} & <0.055 & \text { (unconstrained or LMA) } \\
\tan ^{2} \theta_{13} & <0.075 & \text { (SMA) }
\end{array}
$$

The allowed regions for $\Delta m_{21}^{2}$ and $\theta_{12}$ obtained from this global analysis are shown in Fig.29, both for the unconstrained and constrained case. It is interesting to notice that, in the unconstrained case, the best-fit solution lies in the LMA region.

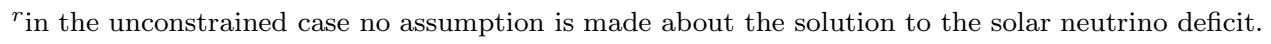




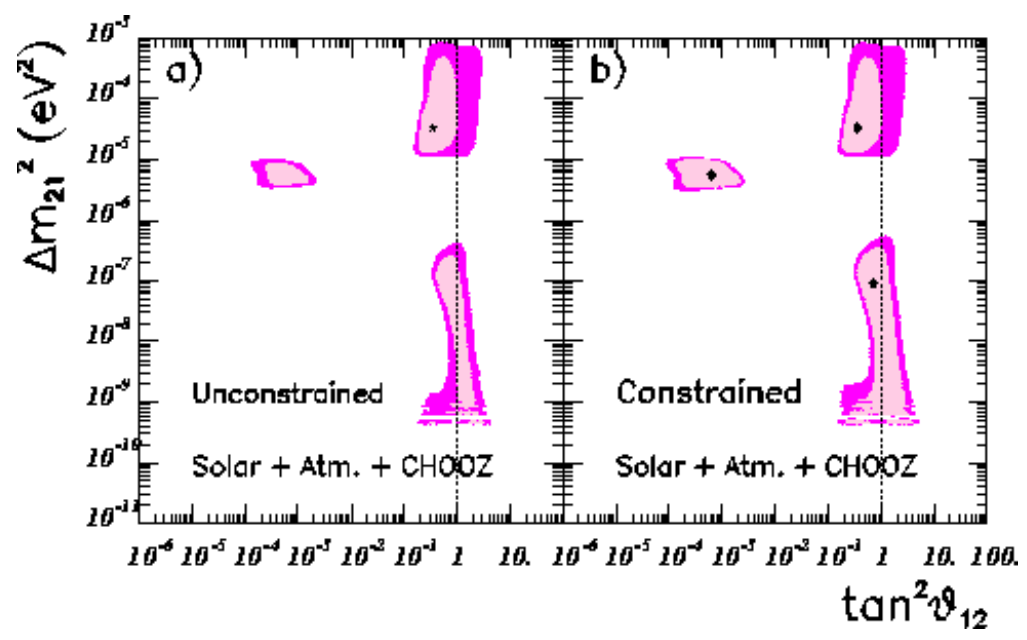

Fig. 29. Allowed regions in the plane $\left\{\tan ^{2} \theta_{12}, \Delta m_{21}^{2}\right.$ as obtained from the global three-neutrino analysis of solar, atmospheric and reactor data of ref 90 , for both the unconstrained (left) and the constrained fit (right). The star denotes the global best fit solution for the unconstrained fit and local best fit points for the constrained fit.

In summary, the analysis in ref.90 shows that, although all solutions to the solar neutrino deficit are still allowed, the LMA solution appears to be favoured. Moreover both solar and atmospheric neutrino data seem to prefer small values of the mixing angle $\theta_{13}$, the constraint becoming more stringent with the inclusion of the $\mathrm{CHOOZ}$ result. Limits have been extracted also on the other relevant oscillation parameters.

Ignoring $\mathrm{CP}$ violation, in the limit $U_{e 3} \sim 0$ (that is to say $s_{13} \sim 0$ and $c_{13} \sim 1$ ) the MNS matrix (13) can be written as:

$$
U \simeq\left(\begin{array}{ccc}
c_{12} & s_{12} & 0 \\
-s_{12} c_{23} & c_{12} c_{23} & s_{23} \\
s_{12} s_{23} & -c_{12} s_{23} & c_{23}
\end{array}\right),
$$

One appealing realization of this matrix would be that corresponding to $b i$ maximal mixing (see, for example, ref.129), which postulates that both atmospheric and solar neutrino anomalies can be interpreted as neutrino oscillations with maximal mixing (that it to say both $\theta_{23}$ and $\theta_{12}$ equals $\pi / 4$ ). In this case:

$$
\left(\begin{array}{ccc}
\frac{1}{\sqrt{2}} & \frac{1}{\sqrt{2}} & 0 \\
-\frac{1}{2} & \frac{1}{2} & \frac{1}{\sqrt{2}} \\
\frac{1}{2} & -\frac{1}{2} & \frac{1}{\sqrt{2}}
\end{array}\right) \text {. }
$$

However it has to be noticed that this form of the mixing matrix seems to be 
incompatible with the LMA solution of the solar neutrino deficit.130

If one tries to define a coherent picture which accommodates also the LSND result, a fourth neutrino has to be introduced. Essentially only two mass schemes can used to describe the mass spectrum, the so called $3+1$ and $2+2$ schemes. The $3+1$ scheme, which seems to be disfavoured by experimental data, 131,132 corresponds to having three relatively close neutrino masses, whose spacing is determined by the two $\Delta m^{2}$ from the solar and atmospheric neutrino analyses, separated by about $1 \mathrm{eV}^{2}$ from an isolated level, which is responsible for the LSND anomaly. In the $2+2$ scheme, instead, two doublets of mass eigenstates, spaced by $\Delta m_{\odot}^{2}$ and $\Delta m_{a t m}^{2}$ respectively, are separated by the LSND gap. Several analyses of the solar and atmospheric neutrino data in the $2+2$ four-neutrino framework have been recently presented, showing that, although Super-Kamiokande data disfavour both the pure

$\nu_{\mu} \rightarrow \nu_{s}$ and the pure $\nu_{\mu} \rightarrow \nu_{e}$ channels, it is not possible to exclude their occurrence with sizable oscillation amplitude.133, 134

\section{Conclusions}

In this paper we have reviewed the current status of the search for neutrino oscillations. After an introduction of the formalism of neutrino oscillation phenomenology, we have discussed the various experimental results which, over the past three decades, more or less convincingly, have provided hints for the existence of flavour mixing in the neutrino sector: the solar neutrino deficit, the atmospheric neutrino anomaly and the LSND effect. If any of these indications will be confirmed by future experiments, we would be forced to extend our description of sub-nuclear phenomena beyond the Standard Model.

Having described the different experimental techniques used to explore different domains of the oscillation parameter space, and having discussed the sources of systematic error intrinsic to each method, we hope we have given most of the information necessary to discern the robustness of each method and result. However, whatever the preference of the reader may be, it is undeniable that more experimental data is urgently needed, before a clearer picture of neutrino oscillation phenomenology can be drawn.

The near and medium term experimental programme, which is supposed to answer many of the as yet unresolved puzzles, has been described in the previous sections. However it is possible that the experiments already approved will not be able to provide a definitive solution to all our pending questions. In particular, none of the experiments scheduled to start running over the next few years will have the sensitivity to address CP violation in the neutrino sector and, except if the actual value of $\theta_{13}$ is very close to the present CHOOZ limit, none of them will be able tg measure that mixing angle. Very ambitious projects, such as neutrino factorie 135 or super-intense neutrino beams (super-beams),136 if ever approved, might hold the key to the ultimate solution to the fascinating neutrino mystery.

To conclude, whatever the outcome of future experiments, there is no doubt this is an extremely exciting time for neutrino physics. 


\section{Acknowledgements}

The author would like to thank J. H. Cobb and A. Weber, for helpful and stimulating discussions during the preparation of this review and for their careful reading of the manuscript, and L. DiLella and C. Yanagisawa, for having kindly provided the OPERA sensitivity plots and the latest Super-Kamiokande figures respectively.

\section{References}

1. F. Reines, C. L. Cowan, Nature 178 (1956) 446.

2. B. Pontecorvo, Zh. Eksp. Teor. Fiz. 33 (1957) 549; Zh. Eksp. Teor. Fiz. 34 (1958) 247.

3. Q. R. Ahmad et al., (the SNO Coll.), nucl-ex/0106015, submitt. to Phys. Rev. Lett..

4. V. M. Lobashev et al., Nucl. Phys. B (Proc. Suppl.) 91 (2001) 280-286.

5. J. Bonn et al., Nucl. Phys. B (Proc. Suppl.) 91 (2001) 273-279.

6. Particle Data Group, D. E. Groom et al., Eur. Phys. J. C15 (2000) 1.

7. J. M. Roney et al., Nucl. Phys. B (Proc. Suppl.) 91 (2001) 287-292.

8. M. Gell-Mann, P. Ramond and R. Slansky, in Supergravity, ed. P. van Niuwenhuizen and D. Z. Freedman (North Holland,Amsterdam, 1979) p.315; T. Yanagida, in Proc. of the Workshop on the Unified Theory and Baryon Number in the Universe, ed. O. Sawada and A. Sugamoto, KEK, Japan (1979) p.95; R. N. Mohapatra and G. Senjanović, Phys. Rev. Lett. 44 (1980) 912.

9. S. A. Bludman, D. C. Kennedy and P. G. Langacker, Phys. Rev. D 45 (1992) 1810; S. A. Bludman, D. C. Kennedy and P. G. Langacker, Nucl. Phys. B 374 (1992) 373.

10. K. Kodama et al., Phys. Lett. B 504 (2001) 218-224

11. G. Giunti and C. W. Kim, hep-ph/0011074 (2000)

12. Z. Maki, M. NAkagawa, S. Sakata, Progr. Theor. Phys. 28 (1962) 870.

13. L. Wolfenstein, Phys. Rev. D 17 (1978) 2369.

14. S. P. Mikheyev and A. Yu. Smirnov, Yad. Fiz. 42 (1985) 1441.

15. R. Davis, Jr., D. S. Harmer and K. C. Hoffman, Phys. Rev. Lett. 20 (1968) 1205.

16. R. Davis, Jr., Prog. Part. Nucl. Phys. 32 (1994) 13; B. T. Cleveland, T. Daily, R. Davis, Jr., J. R. Distel, K. Lande, C. K. Lee, P. S. Wildenhain and J. Ullman, Astrophys. J. 496 (1998) 505; K. Lande et al., in the Proc. of the XIX Intern. Conf. on Neutr. Phys. \& Astr., Sudbury, Canada, Nucl. Phys. B (Proc. Suppl.) 91 (2001) 50-57.

17. A. I. Abazov et al., Phys. Rev. Lett. 67 (1991) 3332; V. Gavrin et al., in the Proc. of the XIX Intern. Conf. on Neutr. Phys. \& Astr., Sudbury, Canada, Nucl. Phys. B (Proc. Suppl.) 91 (2001) 36-43.

18. P. Anselmann et al., Phys. Lett. B 285 (1992) 376; W. Hampel et al., Phys. Lett. B 388 (1996) 384; W. Hampel et al., Phys. Lett. B 447 (1999) 127.

19. K. S. Hirata et al., Phys. Rev. D 38 (1988) 448; K. S. Hirata et al., Phys. Rev. D 44 (1991) 2241; K. S. Hirata et al., Phys. Rev. Lett. 77 (1996) 1683.

20. Y. Suzuki, Talk given at the 19th Intern. School of Nucl. Phys. - Neutrinos in Astro, Part. and Nucl. Phys., Erice, Italy, published in Prog. Part. Nucl. Phys. 40 (1998) 427-441; Y. Fukuda et al., Phys. Rev. Lett. 81 (1998) 1158.

21. J. N. Bahcall and C. A. Barnes, Phys. Lett. 12 (1964) 48.

22. J. N. Bahcall, S. Basu and M. H. Pinsonneault, Phys. Lett. B 433 (1998) 1-8.

23. J. N. Bahcall, M. H. Pinsonneault and S. Basu, astro-ph/0010346

24. S. M. Bilenky et al., Prog. Part. Nucl. Phys. 43 (1999) 1-86.

25. J. N. Bahcall's home page, http://www.sns.ias.edu/ jnb.

26. S. Basu et al., Mon. Not. R. Astron. Soc. 292 (1997) 1402.

27. M. Altmann et al., Phys. Lett. B 490 (2000) 16-26. E. Bellotti et al., in the Proc. of 
the XIX Intern. Conf. on Neutr. Phys. \& Astr., Sudbury, Canada, Nucl. Phys. B (Proc. Suppl.) 91 (2001) 44-49.

28. W. Hampel et al., Phys. Lett. B 420 (1998) 114;

29. W. Hampel et al., Phys. Lett. B 436 (1998) 158.

30. D. N. Abdurashnitov et al., Phys. Rev. Lett. 77 (1996) 4708.

31. K. S. Hirata et al., Phys. Lett. B 220 (1989) 308.

32. S. Fukuda et al., Phys. Rev. Lett. 86 (2001) 5651-5655; S. Fukuda et al., Phys. Rev. Lett. 86 (2001) 5656-5660.

33. M. Nakahata et al., Nucl. Instr. Meth. A 421 (1999) 113-129.

34. E. Blaufuss, hep-ex/000501, LSU-HEPA-1-00, Baton Rouge, Louisiana State Univ., May 2000, submitt. to Nucl. Instr. Meth. A

35. J. N. Bahcall, P. I. Krastev and A. Yu. Smirnov, Phys. Rev. D 58 (1998) 096016.

36. A. Dar and G. Shaviv, Phys. Rep. 311 (1999) 115-141.

37. A. de Gouvêa, A. Friedland and H. Murayama, Phys. Lett. B 490 (2000) 125-130.

38. A. B. McDonald, SNO Coll., in the Proc. of the XIX Intern. Conf. on Neutr. Phys. \& Astr., Sudbury, Canada, Nucl. Phys. B (Proc. Suppl.) 91 (2001) 21-28.

39. G. Ranucci et al., in the Proc. of the XIX Intern. Conf. on Neutr. Phys. \& Astr., Sudbury, Canada, Nucl. Phys. B (Proc. Suppl.) 91 (2001) 58-65.

40. A. Piepke, KamLAND Coll., in the Proc. of the XIX Intern. Conf. on Neutr. Phys. \& Astr., Sudbury, Canada, Nucl. Phys. B (Proc. Suppl.) 91 (2001) 58-65.

41. G. L. Fogli et al., Phys. Rev. D 63 (2001) 113016; F. L. Villante et al., Phys. Rev. D 59 (1999) 013006.

42. J. N. Bahcall and P. Krastev, Phys. Rev. C 56 (1997) 2839; A. de Gouvêa, A. Friedland and H. Murayama, hep-ph/9910280.

43. F. Arneodo et al., LNGS-P28/2001, LNGS-EXP 13/89 add.1/01, ICARUS-TM/200103, hep-ex/0103008 (2001).

44. Web page: http://www.physics.brown.edu/research/heron/index.htm

45. Web page: http://sg1.hep.fsu.edu/hellaz/

46. T. K. Gaisser, in the Proc. of the VIth Intern. Workshop on Topics in Astrop. and Undergr. Phys., Paris, France, Nucl. Phys. B (Proc. Suppl.) 87 (2000) 145-151.

47. Plenary talk at the XIX International Symposium on Lepton and Photon Interactions at High Energies, Stanford, 1999, TUHEP-99-04, PDK-741, hep-ex/9912007

48. T. J. Heines et al, Phys. Rev. Lett 57 (1986) 1986; R. Becker-Szendy et al., Phys. Rev. D 46 (1992) 3270; D. Caspar et al., Phys. Rev. Lett. 66 (1991) 2561; R. Becker-Szendy et al., Nucl. Phys. B (Proc. Suppl.) 38 (1995) 331.

49. K. S. Hirata et al., Phys. Lett. B 205 (1988) 416; K. S. Hirata et al., Phys. Lett. B 280 (1992) 146; Y. Fukuda et al., Phys. Lett. B 335 (1994) 237-245.

50. M. Aglietta et al., Europhys. Lett. 8 (1989) 611.

51. C. Barger et al., Phys. Lett. B 227 (1989) 489; C. Barger et al., Phys. Lett. B 245 (1990) 305; K. Daum et al., Z. Phys. C 66 (1995) 417.

52. J. Engel, E. Kolbe, K. Langanke and P. Vogel, Phys. Rev. D 48 (1993) 3048.

53. W. W. M. Allison, Phys. Lett. B 391 (1997) 491-500.

54. Y. Fukuda et al., Phys. Lett. B 235 (1994) 237.

55. S. P. Mikheyev, in the Proceedings of the VIIIth Rencontres de Blois - Neutrinos, Dark Matter and the Universe, Blois, France, June 1996.

56. S. Ahlen et al., Phys. Lett. B 357 (1995) 481-486.

57. Y. Fukuda et al., Phys. Lett. B 433 (1998) 9-18; Y. Fukuda et al., Phys. Lett. B 436 (1998) 33-41;

58. T. Futagami et al., Phys. Rev. Lett. 82 (1999) 5194.

59. H. Sobel et al., in the Proc. of the XIX Intern. Conf. on Neutr. Phys. \& Astr., Sudbury, Canada, Nucl. Phys. B (Proc. Suppl.) 91 (2001) 127-133. 
60. R. Bellotti et al., Phys. Rev. D 60 (1999) 052002.

61. M. Boezio et al., Phys. Rev. Lett. 82 (1999) 4757; M. Boezio et al., Phys. Rev. D 62 (2000) 032007.

62. S. Coutu et al., Phys. Rev. D 62 (2000) 032001.

63. J. Alcaraz et al., Phys. Lett. B 490 (2000) 27-35.

64. S. Orito et al., Phys. Rev. Lett. 84 (2000) 1078; T. Sanuki et al., Ap. J. 545 (2000) 1135.

65. M. Boezio et al., Ap. J. 518 (1999) 457-472.

66. J. W. Mitchell et al., Phys. Rev. Lett. 76 (1996) 3057.

67. M. S. Longair, High Energy Astrophysics, (Cambridge University Press, 1981, Reprinted 1997), Vol. 1, pp.300-324.

68. M. A. Shea and D. F. Smart, in 19th Intl. Conf. Cosmic Rays, La Jolla, USA, Vol. 4, p.501.

69. W. R. Webber and J. A. Lezniak, Astrohys. Space Sci. 30 (1974) 361.

70. M. Honda et al., Phys. Lett. B 248 (1990) 193; M. Honda et al., Phys. Rev. D 52 (1995) 4985.

71. G. Barr, T. K. Gaisser and T. Stanev, Phys. Rev. D 39 (1989) 3532; V. Agrawal et al., Phys. Rev. D 53 (1996) 1314.

72. G. Battistoni, in the Proc. of Europhysics Neutrino Oscillation Workshop - NOW 2000, Lecce, Italy, Nucl. Phys. B (Proc. Suppl.) 100 (2001) 101-106.

73. G. Battistoni et al., Astrop. Phys. 12 (2000) 315.

74. P. Lipari, Astrop. Phys. 14 (2000) 153.

75. M. Honda et al., hep-ph/0103328.

76. A. Fassò, A. Ferrari, J. Ranft and P. R. Sala, Web page http://www.cern.ch/fluka .

77. M.G. Catanesi et al., CERN-SPSC/99-35, SPSC/P315 (1999).

78. M. G. Catanesi et al., CERN-SPSC/2001-017, SPSC/P322, CERN, May 2001.

79. S. Afanasiev et al., Nucl. Instrum. Meth. A 430 (1999) 210.

80. C. P. Achenbach and J. H. Cobb, to appear in the Proceedings of the 27th International Cosmic Ray Conference (ICRC) 2001, Hamburg, Germany, 2001.

81. S. Kasuga et al., Phys. Lett. B 374 (1996) 238-242.

82. R. Engel, T. K. Gaisser, T. Stanev, Phys. Lett. B 472 (2000) 113-118.

83. T. Toshito, in XXXVIth Rencontres de Moriond, Electroweak Interactions and Unified Theories, Les Arcs, Savoie, France (March 2001).

84. C. Yanagisawa (for the Super-Kamiokande Coll.), in Frontiers in Contemporary Physics 2001, Vanderbilt University, Nashville, Tennessee, USA (March 2001).

85. T. Mann et al., in the Proc. of the XIX Intern. Conf. on Neutr. Phys. \& Astr., Sudbury, Canada, Nucl. Phys. B (Proc. Suppl.) 91 (2001) 134-140.

86. M. Spurio (MACRO Coll.), in 3rd International Workshop on New Worlds in Astroparticle Physics, Faro, Portugal, Sep 2000, DFUB-2001-1, hep-ex/0101019

87. K. Scholberg, in DPF 2000: The Meeting of the Division of Particles and Fields of the American Physical Society, Columbus, Ohio, Aug 2000, subm. to Int. J. Mod. Phys. A, hep-ex/001102才.

88. M. Apollonio et al., Phys. Lett. B 466 (1999) 415-430.

89. F. Boehm et al., Nucl. Phys. B (Proc.Suppl.) 77 (1999) 166-170.

90. M. C. Gonzalez-Garcia et al., Phys. Rev. D 63 (2001) 033005.

91. P. Lipari and M. Lusignoli, Phys. Rev. D 58 (1998) 073005.

92. S. Fukuda et al., Phys. Rev. Lett. 85 (2000) 3999-4003.

93. K. Nakamura et al., in the Proc. of the XIX Intern. Conf. on Neutr. Phys. \& Astr., Sudbury, Canada, Nucl. Phys. B (Proc. Suppl.) 91 (2001) 203-209; S. Ahn et al., hepex/0103001, Submitt. to Phys. Rev. Lett.

94. N. Y. Agafonova et al., LNGS P26/2000, CERN/SPSC 2000-031, SPSC/M657 (2000). 
95. C. Yanagisawa (for the K2K Coll.), in Frontiers in Contemporary Physics 2001, Vanderbilt University, Nashville, Tennessee, USA (March 2001).

96. FERMILAB-PROPOSAL-P-875 (1995); E. Abbles et al., FERMILAB-PROPOSALP-875-ADD, NuMI-L-79 (1995); P. Adamson et al., The MINOS Detectors Technical Design Report, NuMI-L-337 (1998).

97. D. A. Crane et al., FERMILAB-TM-1946, NuMI-B-92; K. Anderson et al., NuMI Facility Technical Design Report, NuMI-346 (1998)

98. G. Acquistapace et al., CERN 98-02, INFN/AE-98/05 (1998); R. Bailey et al. CERNSL/99-034(DI), INFN/AE-99/05 (1999).

99. M. Guler et al., CERN/SPSC 2000-028, SPSC/P318, LNGS P25/2000 (2000).

100. F. Arneodo et al., LNGS-P21/99, INFN/AE-99-17, CERN/SPSC 99-25,SPSC/P314 (1999).

101. J. Thomas et al., CERN-SPSC-2000-046 ; SPSC-M-659 (2000).

102. J. H. Cobb, NuMI-L-272 (1997).

103. D. Petyt NuMI-L-481 (1999); D. Petyt NuMI-L-612 (2000).

104. P. J. Litchfield and D. Petyt, NuMI-L-436 (1999).

105. L. Wai and B. Patterson, hep-ph/010109, NUMI-L-713 (2001); M. Diwan et al., NUMI$L-714$ (2001).

106. J. Altegoer et al.Nucl. Instrum. Meth. A 404 (1998) 96.

107. E. Eskut et al., Nucl. Instrum. Meth. A 401 (1997) 7.

108. S. Aoki (for the OPERA Coll.), in Frontiers in Contemporary Physics 2001, Vanderbilt University, Nashville, Tennessee, USA (March 2001).

109. Web page: http://www.aquila.infn.it/icarus/.

110. ICARUS Coll., Add. to proposal, LNGS-95/10 (1995).

111. E. Scapparone, in the Proceedings of 5th International Workshop on Tau Lepton Physics (TAU 98), Santander, Spain (1998), Nucl.Phys.Proc.Suppl.76 (1999) 471-476; G. Osteria, Nucl.Phys.Proc.Suppl. 78 (1999) 401-406.

112. J. Altegoer et al. Phys. Lett. B 431 (1998) 219-236; P. Astier et al. Phys. Lett. B 453 (1999) 169-186; P. Astier et al. Phys. Lett. B 483 (2000) 387-404.

113. A. Rubbia, in the Proc. of the XIX Intern. Conf. on Neutr. Phys. \& Astr., Sudbury, Canada, Nucl. Phys. B (Proc. Suppl.) 91 (2001) 223-229, hep-ex/0008071.

114. S. Navas-Concha (for the ICARUS Coll.), in Frontiers in Contemporary Physics 2001, Vanderbilt University, Nashville, Tennessee, USA (March 2001).

115. C. Athanassopoulos et al., Nucl. Instr. and Meth. A 388 (1997) 149-172.

116. C. Athanassopoulos et al., Phys. Rev. Lett. 75 (1995) 2650.

117. C. Athanassopoulos et al., Phys. Rev. Lett. 77 (1996) 3082; C. Athanassopoulos et al., Phys. Rev. C 54 (1996) 2685.

118. C. Athanassopoulos et al., Phys. Rev. Lett. 81 (1998) 1774; C. Athanassopoulos et al., Phys. Rev.C 58 (1998) 2489.

119. A. Aguilar et al., hep-ex/0104049 (2001).

120. K.Eitel, in the Proc. of the XIX Intern. Conf. on Neutr. Phys. \& Astr., Sudbury, Canada, Nucl. Phys. B (Proc. Suppl.) 91 (2001) 191-197.

121. B. Achkar et al., Nucl. Phys. B 434 (1995) 503.

122. A. Romosan et al., Phys. Rev. Lett. 78 (1997) 2912.

123. A. De Santo, in the Proceedings of the XXXIInd Rencontres de Moriond, Electroweak Interactions and Unified Theories (1997) 291.

124. G. Drexlin et al., Nucl. Instrum. Meth. A 289 (1990) 490.

125. K. Eitel, New J. Phys. 2 (2000) 1.1.

126. E. Church et al., LA-UR-98-352, FNAL-P-898.

127. A. O. Barzako, in the Proc. of the XIX Intern. Conf. on Neutr. Phys. \& Astr., Sudbury, Canada, Nucl. Phys. B (Proc. Suppl.) 91 (2001) 210-215. 
128. G. L. Fogli, E. Lisi and D. Montanino, Phys. Rev. D 54 (1996) 2048; G. L. Fogli, E. Lisi, A. Marrone and G.Scioscia, Phys. Rev. D 59 (1999) 033001.

129. H. Fritzsch and Z.-Z. Xing, Progr. Part. Nucl. Phys. 45 (2000) 1-81.

130. C. Giunti, Phys. Rev. D 59 (1999) 077301.

131. S. M. Bilenky, C. Giunti and W. Grimus, Eur. Phys. J. C 1 (1998) 247; S. M. Bilenky, C. Giunti, W. Grimus and T. Schwetz, Phys. Rev. D 60 (1999) 073007.

132. V. Barger, S. Pakvasa, T. J. Weiler and K. Whisnant, Phys. Rev. D 58 (1998) 093016.

133. 2048; G. L. Fogli, E. Lisi and A. Marrone, Phys. Rev. D 63 (2001) 053008.

134. M.C. Gonzalez-Garcia, M. Maltoni and C. Pena-Garay, CERN-TH/2001-133, IFIC/0127, hep-ph/0105269 (2001).

135. http://psux1.kek.jp/ nufact01/index.htm.

136. http://www.fnal.gov/projects/muon_collider/nu/study/superbeams/superbeams.htm. 